\title{
NATURE ET CONTRÔLE DES REJETS
}

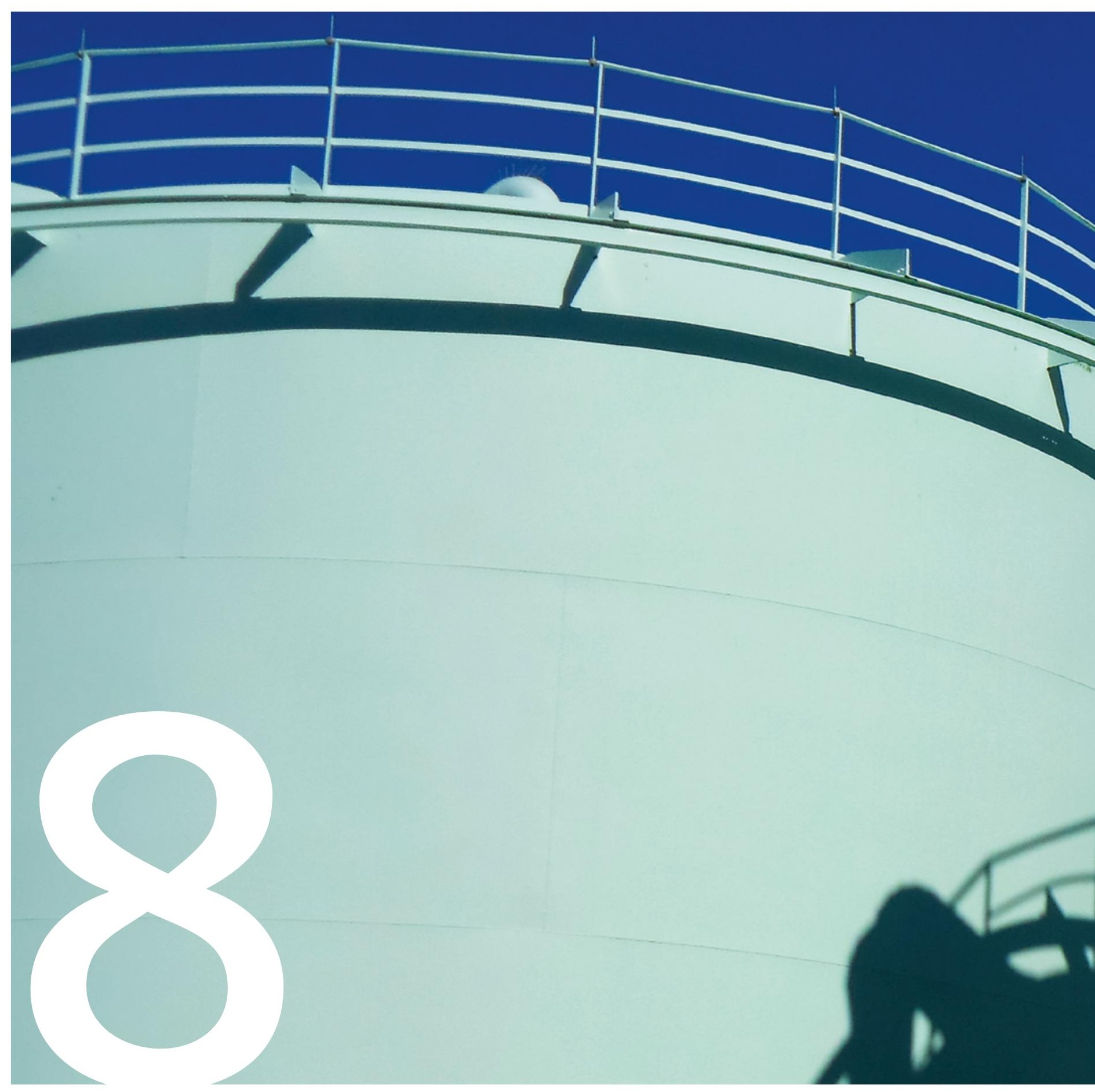




\section{INTRODUCTION}

2. INSTALLATIONS DE COLLECTE, DE TRAITEMENT ET DE REJET DES EFFLUENTS

\section{DOMAINE RADIOACTIF}

3.1 Origine de la radioactivité des effluents rejetés

3.2 Effluents radioactifs gazeux

3.3 Effluents radioactifs liquides

3.4 Eaux d'exhaure des salles des machines

3.5 Rejets gazeux diffus

\section{DOMAINE CHIMIQUE}

4.1 Origine des substances chimiques rejetées

4.2 Collecte et traitement des effluents chimiques liquides

4.3 Rejets chimiques liquides

4.4 Rejets gazeux non radioactifs

\section{REJETS THERMIQUES}

5.1 Caractéristiques des rejets thermiques en circuit ouvert

5.2 Caractéristiques des rejets thermiques en circuit dit "fermé »

5.3 Rejets thermiques

\section{UTILISATION DES EAUX TIÈDES}

6.1 Objectif

6.2 Aspects réglementaires et applications

Annexes

Annexe 8.1 Fonctionnement d'un réacteur à eau pressurisée

Annexe 8.2 Installation de collecte et de traitement des effluents radioactifs liquides et gazeux

Annexe 8.3 Modalités de rejets des effluents radioactifs liquides - Site sur cours d'eau

Annexe 8.4 Modalités de rejets des effluents radioactifs liquides - Site marin

Annexe 8.5 Comptabilisation des activités rejetées

Annexe 8.6 Fonctionnement d'un aéroréfrigérant

Annexe 8.7 Limites de rejets thermiques des centrales EDF mentionnées dans les décisions ASN 


\section{Introduction}

L'exploitation d'une centrale nucléaire entraîne la production d'effluents radioactifs, chimiques et thermiques dont les rejets dans l'environnement sont strictement réglementés (cf. figure 1).

Chaque centrale est équipée de dispositifs de collecte, de traitement et de contrôle des effluents avant rejet. Par ailleurs, une organisation est mise en œuvre afin d'assurer une gestion des effluents dite « optimisée » visant notamment à :

- réduire à la source la production d'effluents, notamment par le recyclage ;

- éliminer les rejets des substances radioactives ou chimiques au moyen de traitements appropriés ; - valoriser, si possible, les résidus de traitement.

\section{Rejets radioactifs}

Le réacteur nucléaire est le siège de la formation de produits radioactifs (produits de fission, produits d'activation, actinides) dont seule une infime partie se retrouve dans les effluents gazeux et liquides rejetés dans l'environnement. Qu'ils soient rejetés par voie atmosphérique (à la cheminée) ou par voie liquide (vidange de réservoirs), les effluents radioactifs sont systématiquement collectés et traités selon leur nature afin de retenir l'essentiel de leur radioactivité. Leur rejet est contrôlé par des analyses préalables ainsi qu'au moyen de dispositifs de mesure de la radioactivité en continu pendant le rejet.

Depuis la mise en exploitation des premières centrales dans les années 1980, des améliorations ont été apportées aux systèmes de collecte et de traitement des effluents, et une gestion optimisée a été mise en œuvre tant en phase de fonctionnement qu'en phase d'arrêt pour maintenance ou renouvellement du combustible.

Par ces actions conjuguées, les rejets d'activité de gaz rares ont été réduits de plus d'un facteur 50 et les rejets liquides hors tritium et carbone 14 l'ont été d'un facteur 100. Les rejets radioactifs ont ainsi atteint un niveau qualifié de "plancher » traduisant la volonté de l'exploitant d'agir pour réduire les rejets d'effluents "aussi bas que raisonnablement possible, compte tenu des aspects économiques et sociaux », en vertu du principe d'optimisation (cf. chapitres 5 et 9).

\section{Rejets chimiques}

Les substances chimiques rejetées par une centrale nucléaire se classent en deux catégories :

- les substances associées aux effluents radioactifs liquides issus du circuit primaire et des circuits auxiliaires nucléaires ainsi que les eaux d'exhaure des salles des machines (circuit secondaire);
- les produits provenant des autres circuits non nucléaires (circuit de refroidissement des condenseurs, station de déminéralisation, station d'épuration...).

Dans le premier cas, il s'agit de substances utilisées pour le contrôle de la réaction nucléaire (acide borique) ou pour le conditionnement chimique des circuits afin de les protéger de la corrosion (lithine, hydrazine, morpholine, ammoniaque, éthanolamine, phosphates...).

Dans le second cas, les substances en question sont issues du traitement antitartre des circuits de refroidissement (sulfates', polyacrylates) et des sous-produits issus du traitement biocide (oxydants résiduels, nitrates, nitrites, $\mathrm{AOX}^{2}$, $\mathrm{THM}^{3}$ pour les sites en bord de rivière; oxydants résiduels et substances organohalogénées bromées pour les sites en bord de mer). À cela s'ajoutent, pour les centrales concernées, les rejets de cuivre et de zinc dus à l'usure des tubes en laiton des condenseurs.

Pour limiter les rejets chimiques, les substances peuvent être recyclées (cas de l'acide borique), voire éliminées en partie par traitement (cas de l'hydrazine). Dans le cas contraire, elles sont rejetées conformément aux dispositions réglementaires.

Par ailleurs, le fonctionnement d'une centrale produit également des rejets chimiques gazeux non radioactifs provenant essentiellement du circuit secondaire (ammoniac, hydrazine...), mais également des installations de combustion de secours et des traitements biocides des circuits de refroidissement.

\section{Rejets thermiques}

Le fluide " eau-vapeur » du circuit secondaire d'une centrale nucléaire suit un cycle thermodynamique dit cycle de Carnot. Au cours du cycle, le fluide « eau-vapeur » échange de l'énergie thermique avec deux sources de chaleur:

- l'une " chaude » constituée de l'eau du circuit primaire dont la température varie en fonctionnement de $286^{\circ} \mathrm{C}$ à $323^{\circ} \mathrm{C}$;

- l'autre "froide » assurée par l'eau du circuit tertiaire de refroidissement du condenseur dont la température peut varier entre zéro et une trentaine de degrés Celsius.

L'eau brute assurant le refroidissement du condenseur est prélevée soit en mer, soit en rivière, et peut être utilisée :

- en circuit ouvert dissipant toute l'énergie thermique extraite au condenseur :

- en circuit dit «fermé » où l'eau brute du circuit tertiaire est à son tour refroidie par un aéroréfrigérant.

1. En 2015-2016, le traitement à l'acide chlorhydrique a été remplacé par un traitement à l'acide sulfurique sur le CNPE de Cattenom.

2. AOX composés organohalogénés adsorbables sur charbon actif.

3. THM composés organohalogénés volatils (notamment chloroforme). 
Les centrales refroidies en circuit ouvert sont implantées en bord de mer et en bordure de fleuve à gros débit (Rhône). L'échauffement du milieu aquatique est dans ce cas de quelques degrés en aval des rejets après mélange. Alors que les centrales des cours d'eau à faible ou moyen débit (Seine, Loire, Vienne, Garonne, Meuse, Moselle) sont refroidies en circuit dit «fermé », ce qui limite l'échauffement à quelques dixièmes de degrés en aval du rejet après mélange.

Fig. 1 L'exploitation d'une centrale nucléaire entraîne la production d'effluents radioactifs, chimiques et thermiques dont les rejets dans l'environnement sont strictement réglementés. Les schémas de principe ci-dessous présentent les circuits de rejet de ces effluents.

\section{Centrale refroidie en circuit ouvert}

Rejets radioactifs

gazeux

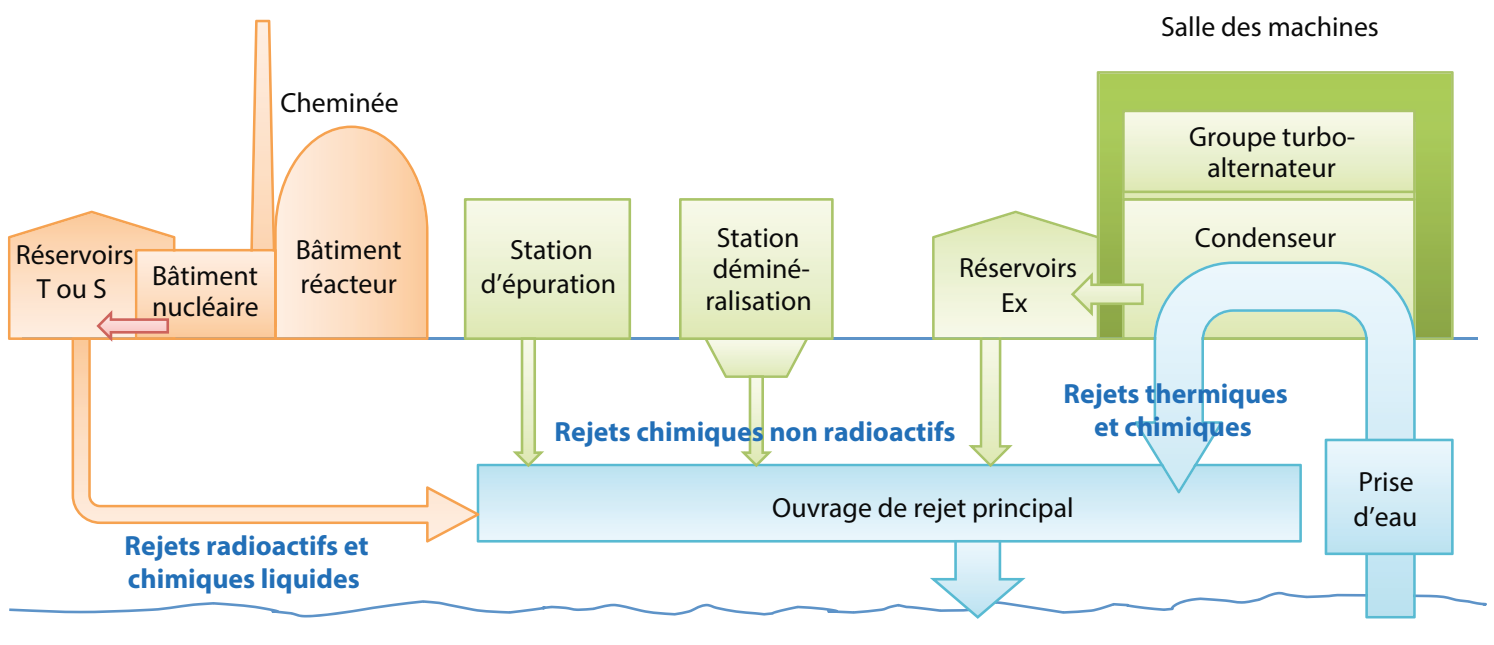

Mer ou rivière

\section{Centrale refroidie en circuit dit " fermé »}

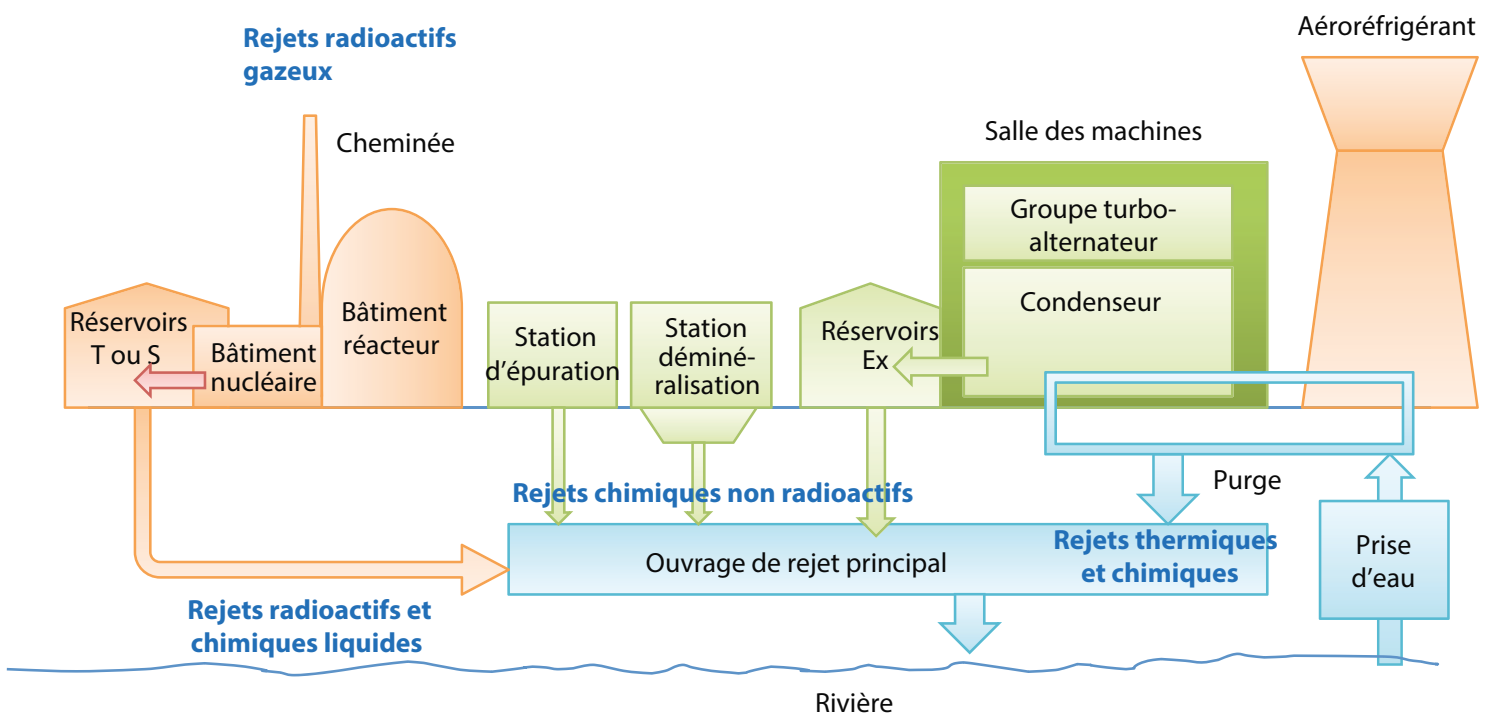




\section{Installations de collecte, de traitement et de rejet des effluents}

Les centrales nucléaires sont conçues pour permettre une collecte sélective des effluents. Celle-ci permet de séparer les effluents selon leur origine et leur composition afin d'adapter le mode de traitement à leurs caractéristiques. Après collecte et traitement éventuel, les effluents sont analysés avant d'être rejetés dans l'environnement selon des règles définies par la réglementation.

Parmi les différents types de rejet, on distingue:

- les rejets radioactifs liquides et gazeux issus des circuits nucléaires (circuit primaire, auxiliaires nucléaires, piscine de désactivation du combustible usé...) qui contiennent également des substances chimiques ;

- les rejets chimiques non radioactifs provenant:

- des salles des machines (circuit « secondaire» renfermant les groupes turbo-alternateur, les postes d'eau...),

- des circuits d'eau brute de refroidissement des condenseurs et des autres circuits de refroidissement (circuit « tertiaire »),
- de la fosse de neutralisation des effluents de la station de déminéralisation,

- de la station d'épuration des eaux usées (eaux vannes),

- des égouts collectant notamment les eaux de pluie ;

- les rejets thermiques liés à l'échauffement de l'eau brute (circuit tertiaire) traversant les tubes de condenseurs et les échangeurs de chaleur.

Si la configuration des circuits d'effluents peut varier selon les paliers de puissance et le mode de refroidissement des condenseurs (cf. annexe 8.2), les principes de conception sont identiques pour toutes les centrales (cf. figure 2). Ceci vaut aussi pour les circuits d'effluents du réacteur EPR, à l'exception toutefois du circuit de traitement des effluents radioactifs gazeux hydrogénés qui présente des particularités (cf. § 3.2).

Fig. 2 Les centrales nucléaires sont conçues pour permettre une collecte sélective des effluents, qui sont ainsi séparés en fonction de leur origine et de leur composition afin d'adapter leur traitement à leurs caractéristiques ; les effluents sont analysés avant d'être rejetés dans l'environnement, selon des règles définies par la réglementation. Le schéma simplifié ci-dessous présente les principes de collecte, traitement et contrôle des effluents.

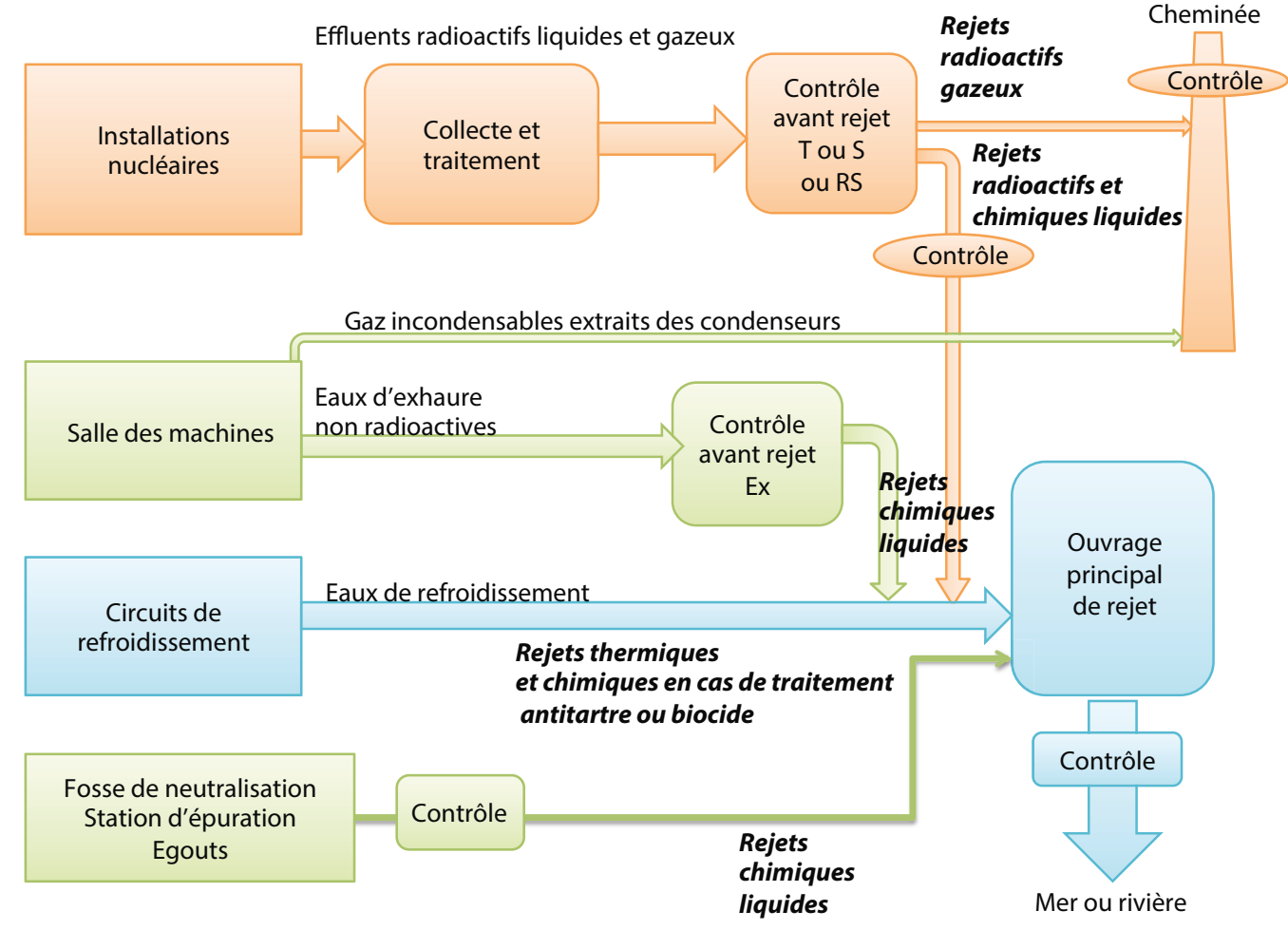




\section{Domaine radioactif}

\subsection{ORIGINE DE LA RADIOACTIVITÉ DES EFFLUENTS REJETÉS}

L'énergie produite par un réacteur nucléaire provient de la fission nucléaire (cf. annexe 8.1). Le réacteur est donc le siège de la formation de substances radioactives (radionucléides) dont seule une infime partie se retrouve dans les effluents gazeux et/ou liquides.

Parmi les substances radioactives susceptibles d'être présentes dans les effluents, on distingue (cf. figure 3) : - les produits créés dans le combustible par fission des atomes d'uranium ou de plutonium. Les radionucléides dits produits de fission (PF), tels que les iodes 131 et 133, les césiums 134 et 137, le krypton 85, le tritium, le carbone 14, le strontium 90 restent en quasi-totalité confinés dans le combustible. Ils peuvent toutefois migrer dans l'eau du circuit pri- maire, en cas d'inétanchéité du gainage du combustible, et donc se retrouver dans les effluents;

- les produits créés à l'extérieur du combustible par l'action des neutrons de fission sur les structures en acier du réacteur (cuve, tuyauteries, grappes de commandes ou sources de neutrons) ainsi que sur les éléments chimiques contenus dans l'eau du circuit primaire, tels que le bore et le lithium. On les appelle les produits d'activation (PA) dont les principaux sont les cobalts 58 et 60 , le manganèse 54, l'antimoine 124, l'antimoine 125, le nickel 63, le chrome 51, l'argent $110 \mathrm{~m}$, mais aussi le tritium et le carbone 14 ;

- les actinides formés dans le combustible par capture de neutrons (américium 241, curium 242, plutonium 239...). Ces radionucléides se caractérisent par l'émission d'un rayonnement alpha. Comme les produits de fission, les actinides restent confinés en quasi-totalité dans le combustible.

Fig. 3 - Parmi les substances radioactives formées par la fission nucléaire au sein du réacteur, on distingue les produits de fission et les actinides (créés dans les crayons de combustible et qui y restent confinés en quasi-totalité) ainsi que les produits d'activation (créés à l'extérieur du combustible et présents dans l'eau du circuit primaire). Seule une infime partie de ces radionucléides se retrouve dans les effluents gazeux et/ou liquides des centrales nucléaires.

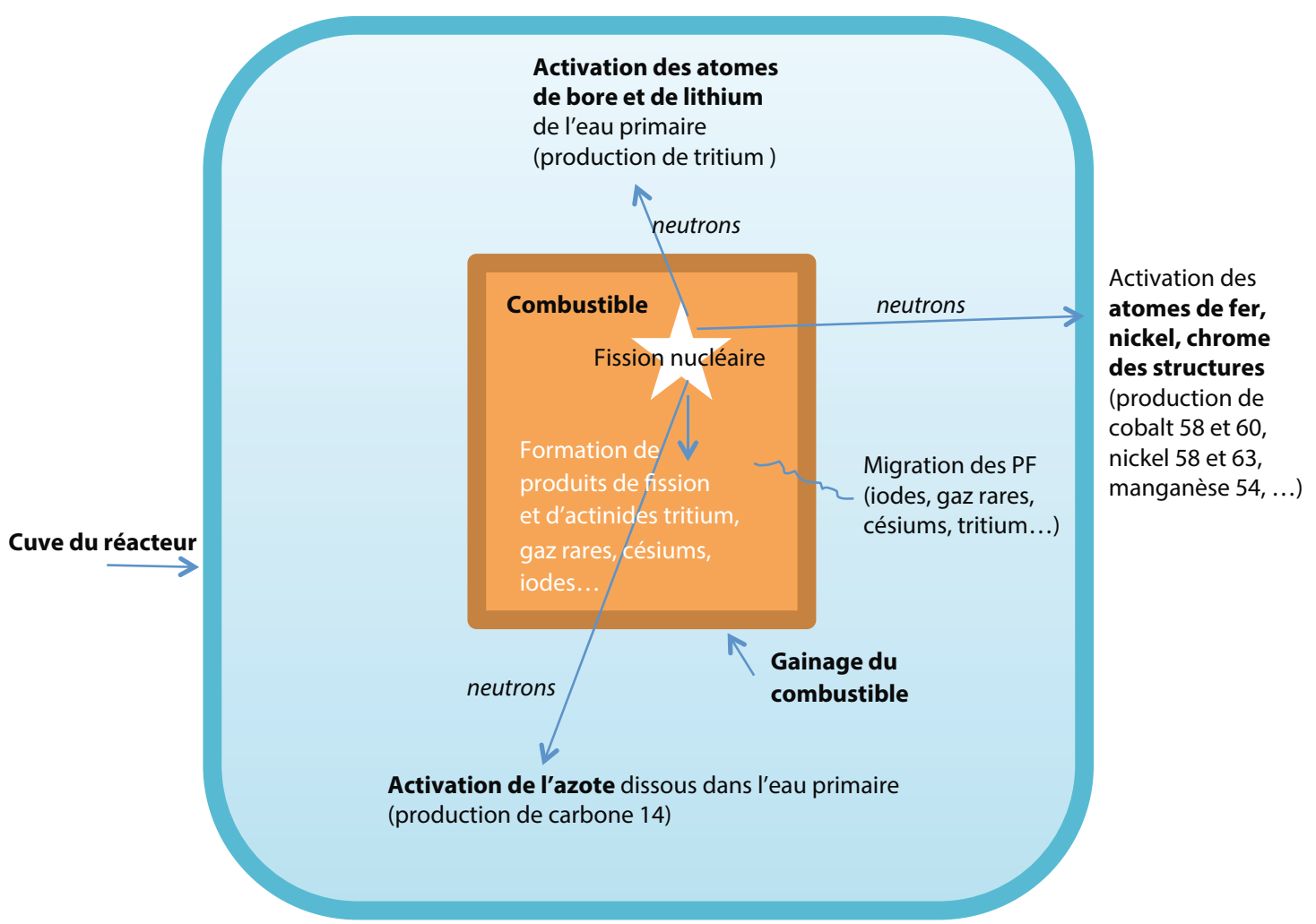




\section{Principaux radionucléides des effluents radioactifs}

Formation des produits de fission (PF)

La fission de l'uranium 235 ou du plutonium 239 engendre généralement la production de deux noyaux de masse intermédiaire appelés produits de fission. Chaque fission dégage une énergie de $200 \mathrm{MeV}$, soit 3,2 × 10-11 Joules (cf. figure 3.1). La fission de $1 \mathrm{~kg}$ de matière produit une énergie thermique de $810^{13}$ Joules, soit l'équivalent de 1900 tonnes de pétrole. Un réacteur de $1000 \mathrm{MWe}$ consomme environ 4 kilogrammes de matières fissiles par jour.

Fig. 3.1 - La fission de l'uranium 235 produit généralement deux produits de fission, de masse plus faible que celle de l'uranium et dégage une énergie importante.
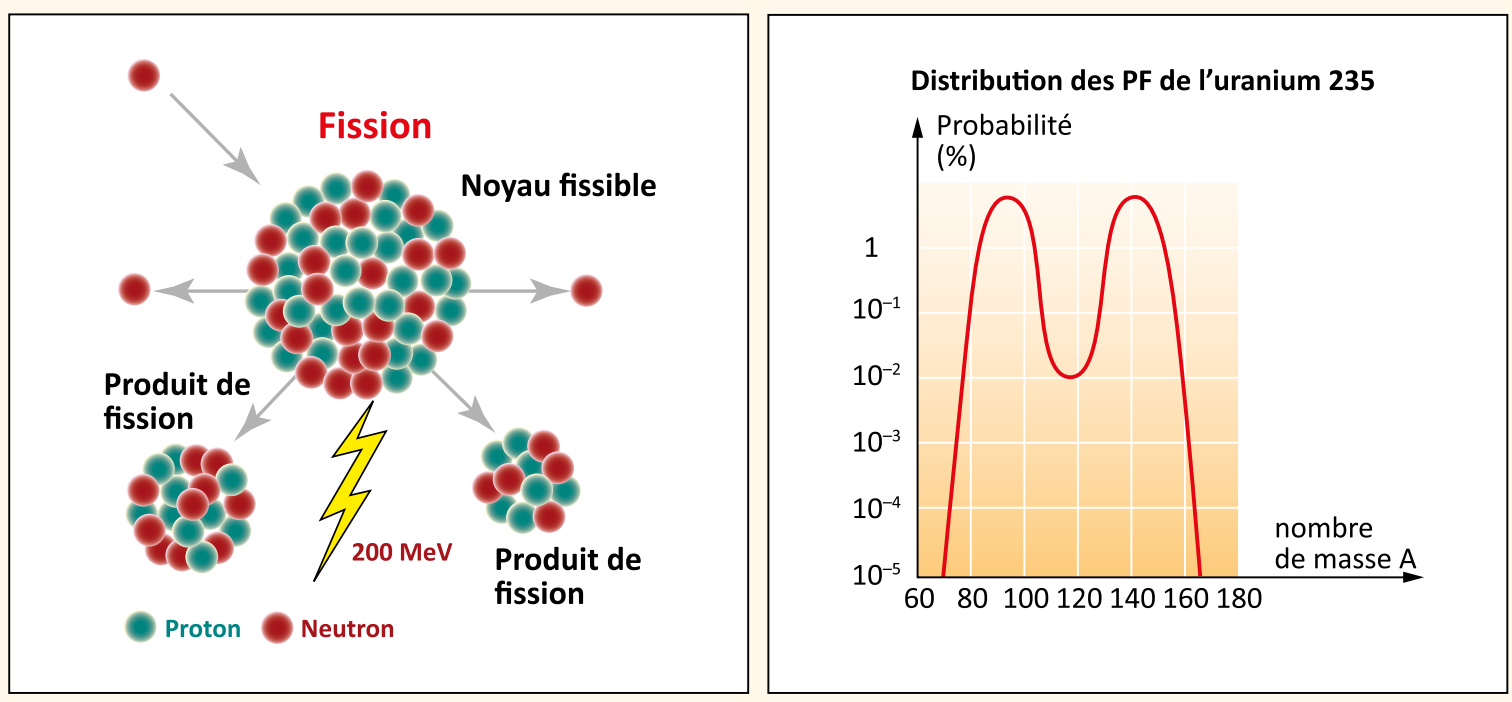

Les produits de fission (PF) sont confinés, en quasi-totalité, dans le combustible nucléaire composé de 40000 à 50000 crayons regroupés en assemblages (cf. figure 3.2).

\section{Principaux PF}

(Légende $: p=$ période radioactive ou demi-vie $; \beta, \gamma=$ type de rayonnement émis ; énergie du rayonnement keV, (\%) pourcentage par désintégration)

\section{Famille des gaz rares}

- Le krypton 85, $p=10,7$ ans, $\beta 687 \mathrm{keV}(99,6 \%), \gamma 514 \mathrm{keV}$ $(0,44 \%)$.

- Le xénon 133, $p=5,3$ jours, $\beta 346 \mathrm{keV}(99,1 \%), \gamma 81 \mathrm{keV}$ (99,8\%).

Famille des halogènes gazeux

- L'iode $131, p=8$ jours, l'iode $132, p=2,3$ heures, I'iode $133, p=20,9$ heures, l'iode $134, p=53$ minutes et l'iode $135, p=6,6$ heures ont des périodes très courtes et émettent des rayonnements $\beta$ et $\gamma$ très énergétiques.

- L'iode 129 produit dans le réacteur reste confiné dans le combustible en quasi-totalité.

Autres radionucléides

- Le césium 134, $p=2,1$ ans et le césium 137, $p=30$ ans émettent des rayonnements $\beta$ et $\gamma$ très énergétiques.

- Le strontium 90, $p=29,1$ ans, émet des rayonnements $\beta$ très énergétiques ; c'est un émetteur $\beta$ pur.

- Le carbone $14, p=5700$ ans, $\beta 156$ keV (100\%), est produit en très faible quantité par fission ternaire. C'est un émetteur dit « $\beta$ pur».

- Le tritium, $p=12,3$ ans, $\beta$ pur d'énergie (18,6 keV), est également formé dans les réacteurs des centrales nucléaires par fission ternaire de certains isotopes d'uranium et de plutonium. Dans le cas des réacteurs à eau sous pression, le tritium reste confiné en quasi-totalité dans le combustible. La diffusion du tritium au travers du gainage en zircaloy est minime $(<<1 \%)$.

Formation des produits d'activation (PA)

Lorsque le noyau d'un atome est frappé par un neutron, ce dernier peut être absorbé par le noyau qui devient instable donc radioactif. Pour retrouver sa stabilité, le noyau va se désintégrer en émettant des rayonnements (cf. figure 3.3). 


\section{Principaux PA}

- Le cobalt $60, p=5,3$ ans, provient du cobalt 59 qui compose certains matériaux inoxydables (portées de robinets utilisés sur le circuit primaire), $\beta 317 \mathrm{keV}(99,9 \%)$, $\gamma 1170$ (99,8\%), $1330 \mathrm{keV}(100 \%)$.

- Le cobalt 58, $p=71$ jours, provient du nickel 58 de l'inconel (alliage présent notamment au niveau des tubes des générateurs de vapeur), $\beta 475 \mathrm{keV}(15 \%), \gamma 811 \mathrm{keV}$ (99,5\%).

- L'antimoine $124, p=60$ jours, provient de l'antimoine 123 qui est présent notamment dans les grappes source de neutrons en alliage d'antimoine et de béryllium. II émet des rayonnements $\beta, 211 \mathrm{keV}(8,7 \%), 611 \mathrm{keV}$ $(51,2 \%), 2302 \mathrm{keV}(23,4 \%)$ et des rayonnements $\gamma_{1}$ $603 \mathrm{keV}(98,2 \%), 1691 \mathrm{keV}(47,5 \%)$.

- L'antimoine $125, p=2,73$ ans, émet des rayonnements $\beta, 767 \mathrm{keV}(77 \%), 622 \mathrm{keV}(23 \%)$ et des rayonnements $\gamma$, $35,5 \mathrm{keV}(88,5 \%), 109,3 \mathrm{keV}(23,1 \%)$.

- L'argent 110 métastable, $p=250$ jours, provient de l'argent 109 présent notamment dans les grappes de commande de la réaction nucléaire en alliage d'argent, d'indium et de cadmium. Il émet des rayonnements $\beta .83 \mathrm{keV}(67,5 \%)$, $530 \mathrm{keV}(30,8 \%)$ et des rayonnements $\gamma$ de $658 \mathrm{keV}(94,7 \%)$ et $885 \mathrm{keV}(74,1 \%)$.

- Le manganèse $54, p=312$ jours, provient du chrome 52 et du chrome 53 présents dans les aciers. II n'émet qu'un rayonnement $\gamma 835 \mathrm{keV}(100 \%)$; c'est un radionucléide dit gamma pur.

- Le fer $59, p=45$ jours, provient du fer 58 et du cobalt 59 des aciers, $\beta 274 \mathrm{keV}(45,2 \%), 466 \mathrm{keV}(53,3 \%)$, et $\gamma 1099 \mathrm{keV}$ $(56,6 \%)$ et $1292 \mathrm{keV}(43,2 \%)$.

- Le chrome 51, p = 28 jours, provient du fer 54, du chrome 50 et du chrome 52 des aciers inoxydables ; c'est un radionucléide dit gamma pur, $\gamma$ de $320 \mathrm{keV}(9,9 \%)$.

- Le nickel 63, p=99 ans, provient du nickel 62 des aciers inoxydables, $\beta 67 \mathrm{keV}(100 \%)$; c'est un radionucléide dit $\beta$ pur.

- Le carbone $14, p=5700$ ans, est essentiellement formé par activation neutronique de l'oxygène 17 et de l'azote 14 contenu dans l'eau du circuit primaire et de l'oxygène 17 dans le combustible. La quantité de carbone 14 formée dépend de l'énergie produite par le réacteur. C'est un émetteur $\beta$ pur de 156 keV (100\%).

- Le tritium, $p=12,3$ ans, est aussi produit par activation neutronique d'éléments légers, tels que le bore 10 et le lithium 6 présents dans le circuit primaire des réacteurs à eau sous pression (environ $86 \%$ pour le bore et $14 \%$ pour le lithium). Dans ce cas, la formation de tritium est liée à l'énergie produite par le réacteur. Le tritium est aussi produit par activation des grappes sources de neutrons utilisées dans les réacteurs du palier 1300 MWe et 1450 MWe. De même propriété chimique que l'hydrogène, il se combine avec l'oxygène pour former notamment de l'eau tritiée (HTO). II n'existe pas de moyens industriels ${ }^{1}$ permettant d'éliminer le tritium contenu dans les effluents. Celui-ci est donc rejeté dans l'environnement par voies liquide et gazeuse. C'est un émetteur $\beta$ pur 18,6 keV (100 \%).

\section{Actinides émetteurs de rayonnement alpha}

La réaction nucléaire s'accompagne aussi de la formation de noyaux lourds sous l'effet des neutrons émis par la fission. Ces noyaux lourds regroupent les actinides majeurs (isotopes de l'uranium et du plutonium) et les actinides mineurs (isotopes du neptunium, de l'américium, et du curium). Les analyses réalisées sur les effluents liquides et gazeux permettent de vérifier l'absence de ces substances dans les rejets.

\section{Unités de la radioactivité}

La radioactivité (propriété de certains corps à se désintégrer en émettant des rayonnements) s'exprime en becquerel (Bq). Le becquerel correspondant à une désintégration par seconde est une unité très petite. On utilise alors des multiples de Bq pour exprimer les activités des radionucléides.

1 kilobecquerel $(1 \mathrm{kBq})=$

1 megabecquerel $(1 \mathrm{MBq})=$

1 gigabecquerel $(1 \mathrm{GBq})=$

$1000000 \mathrm{~Bq}=10^{6} \mathrm{~Bq}$

$1000000000 \mathrm{~Bq}=10^{9} \mathrm{~Bq}$

1 terabecquerel $(1 \mathrm{TBq})=1000000000000 \mathrm{~Bq}=10^{12} \mathrm{~Bq}$

Pour chaque substance, il existe une correspondance entre l'activité exprimée en Bq et la masse en gramme (cf. figure 3.4).
Fig. 3.4 Exemple de correspondance entre l'activité en becquerel (Bq) et la masse en gramme : 0,2 microgrammes d'iode 131 , 3 microgrammes de tritium ou 6 milligrammes de carbone 14 génèrent une activité de 1 gigabecquerel.

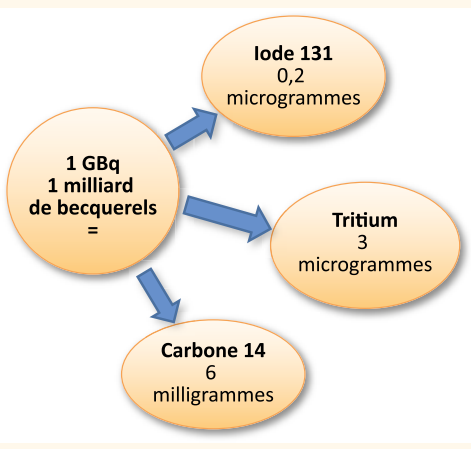

1. Des techniques permettant de concentrer le tritium ont été développées en laboratoire sur des effluents fortement tritiés (plusieurs $\mathrm{TBq} / \mathrm{L}$ ) ; elles ne sont pas applicables aux effluents peu tritiés des centrales nucléaires d'EDF (quelques MBq/L). 


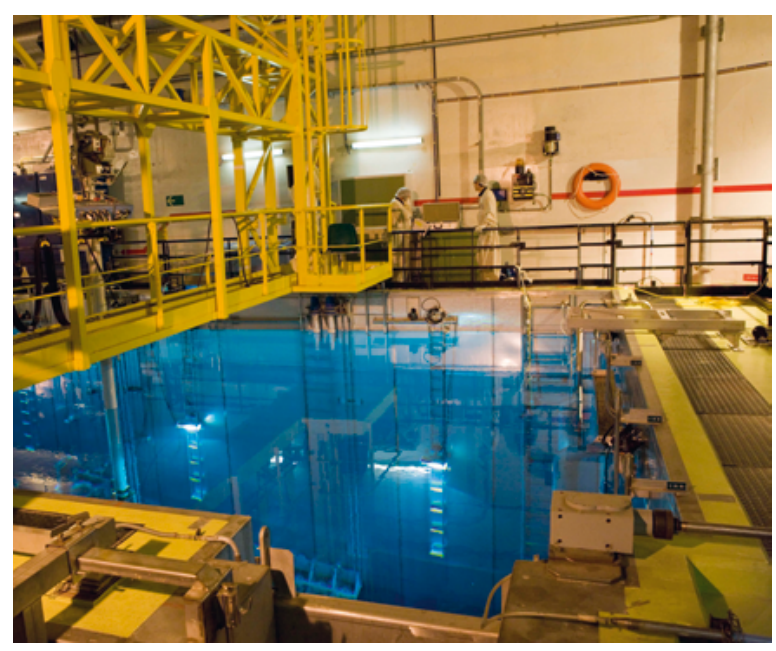

Piscine de désactivation du combustible usé à la centrale de Belleville sur Loire. La piscine où le combustible usé séjourne pendant plusieurs années avant d'être évacué à l'usine de retraitement, est équipée d'un circuit de refroidissement et de purification qui lui est propre - ( ) EDF - Bruno Conty.

\subsection{EFFLUENTS RADIOACTIFS GAZEUX}

\section{Nature, collecte et traitement}

Les effluents radioactifs gazeux se divisent en deux grandes catégories (cf. figure 4):

- les effluents gazeux dits « hydrogénés » proviennent du dégazage des effluents liquides issus du circuit primaire. Ces effluents contiennent de l'hydrogène dissous car l'eau du circuit primaire est volontairement saturée en hydrogène afin d'éviter sa décomposition sous l'effet des rayonnements (radiolyse). La radioactivité des effluents gazeux est due à la présence dans l'eau primaire de produits de fission gazeux (krypton, xénon, iode...) ou de produits d'activation (tritium...).

Afin d'éviter tout mélange avec l'oxygène de l'air, ces effluents hydrogénés sont collectés et stockés dans des réservoirs dénommés RS, préalablement remplis d'azote, gaz inerte. La capacité minimale du stockage est fixée par la réglementation à au moins $2000 \mathrm{Nm}^{3}($ () par paire d'unités sur le palier 900 MWe, au moins $1500 \mathrm{Nm}^{3}$ par unité sur le palier $1300 \mathrm{MWe}$ et au moins $2000 \mathrm{Nm}^{3}$ par unité sur le palier $1450 \mathrm{MWe}$. Les effluents sont entreposés pendant au moins 30 jours, durée réglementaire, pour permettre à la radioactivité de décroître suffisamment avant rejet. Les gaz radioactifs ont pour la plupart des périodes radioactives inférieures à la semaine. Un stockage de 30 jours diminue leur radioactivité initiale d'un facteur 10 au moins.

Ces effluents sont analysés avant leur rejet qui s'effectue par la cheminée après passage sur des filtres absolus à très haute efficacité (THE), pour la rétention des aérosols, et sur des pièges à iodes à charbon actif (PI). L'efficacité des dispositifs de filtration (> 1000 pour les THE ; > 100 pour les pièges à iodes) est testée périodiquement. Ces rejets occasionnels, dits " concertés ", représentent un volume annuel d'environ $2000 \mathrm{Nm}^{3}$ pour une unité de production (entre $1300 \mathrm{Nm}^{3}$ pour le palier $900 \mathrm{MWe}$ et $4100 \mathrm{Nm}^{3}$ pour le palier $1450 \mathrm{MWe}$ ).

Sur le réacteur EPR, la conception des circuits est quelque peu différente. Les effluents " hydrogénés 》 sont directement orientés vers un circuit de traitement spécifique ;

- les effluents gazeux dits « aérés » proviennent:

- de la collecte des évents des circuits de traitement des effluents liquides radioactifs, de la dépressurisation du bâtiment du réacteur dénommé BR,

- de l'air de la ventilation des locaux de l'îlot nucléaire. La ventilation maintient les locaux en légère dépression par rapport à l'extérieur et évite ainsi les fuites de gaz ou de poussières contaminées vers l'environnement.

Ces effluents constituent, en volume, l'essentiel des rejets gazeux radioactifs, soit 1 à 2 milliards de $\mathrm{Nm}^{3}$ par unité et par an.

L'air de ventilation transite par des filtres absolus à très haute efficacité (THE) et, dans certains circuits, sur des pièges à iodes à charbon actif avant d'être rejeté en continu à la cheminée. Ces rejets sont dits « permanents ».

Les opérations de dépressurisation de l'air du bâtiment réacteur (BR), en phase d'arrêt, conduisent à des rejets dits « concertés 》 qui représentent un volume moyen d'environ $180000 \mathrm{Nm}^{3}$ par unité et par an.

Les gaz incondensables extraits des condenseurs des turbo-alternateurs sont susceptibles d'être radioactifs en cas de défaut d'étanchéité des tubes de générateurs de vapeur; les gaz radioactifs et le tritium du circuit primaire pouvant alors migrer vers le circuit secondaire. Ces incondensables sont contrôlés par une chaîne de mesure de la radioactivité en continu et orientés vers la cheminée.

Enfin, l'air de ventilation de certains bâtiments non raccordés à la cheminée (laverie, atelier chaud, bâtiment des auxiliaires de conditionnement des déchets "BAC », laboratoire chaud de chimie, laboratoire "Effluents »...) est filtré avant évacuation par son exutoire spécifique.

\section{Contrôles des effluents radioactifs gazeux} avant rejet (rejets dits concertés)

Les effluents des réservoirs RS et l'air du bâtiment du réacteur sont contrôlés avant rejet. Les analyses sont effectuées au laboratoire "Effluents » sur des prélèvements d'air et d'aérosols et portent notamment sur l'activité $\beta$ globale et l'analyse des constituants. Par ailleurs, il est contrôlé que ces effluents ne contiennent pas d'émetteurs alpha d'origine artificielle dont le rejet n'est pas autorisé ${ }^{2}$ (cf. chapitre $11 \S 3.2$ ).

1. $\mathrm{Nm}^{3}$ : normal mètre cube ; volume de gaz déterminé aux conditions normales de température et de pression.

2. L'arrêté du 14 juin 2017 portant homologation de la décision n² 2017-DC-0588 de l'ASN du 06 avril 2017 précise que la mesure de l'activité al pha globale d'origine artificielle doit être réalisée par une méthode garantissant un seuil de décision ne dépassant pas $1.10^{-3} \mathrm{~Bq} / \mathrm{m}^{3}$. 
Fig. 4 Les effluents radioactifs gazeux des centrales nucléaires se divisent en deux grandes catégories : les effluents hydrogénés (qui proviennent du dégazage des effluents liquides du circuit primaire) et les effluents aérés (qui proviennent des évents des circuits de traitement des effluents radioactifs liquides, de la dépressurisation du bâtiment réacteur et de l'air de ventilation des locaux de l'îlot nucléaire). Ces effluents sont traités et contrôlés avant rejet, selon le schéma de principe ci-dessous.

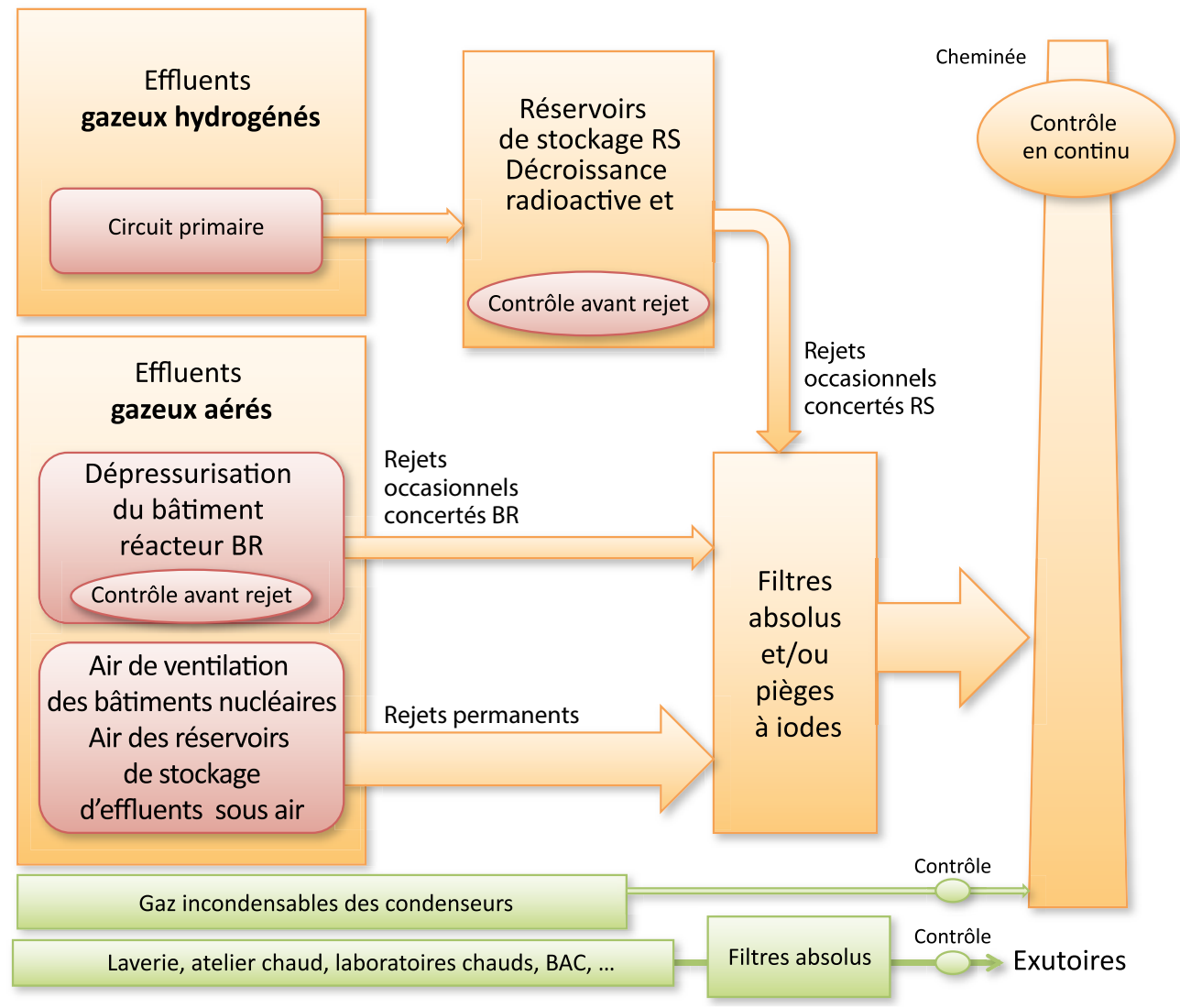

Contrôles des rejets gazeux à la cheminée Chaque cheminée de rejet est instrumentée pour permettre les contrôles des rejets radioactifs gazeux (cf. chapitre 11 \& 3.2).

Les contrôles des rejets sont réalisés en permanence par:

- une mesure enregistrée en continu de la radioactivité bêta globale de l'air rejeté au moyen de deux appareils redondants, dont les alimentations électriques sont secourues en cas de perte de la source électrique principale. Ces appareils retransmettent la mesure et une alarme en salle de commande en cas de dépassement d'un seuil fixé réglementairement à $4 \mathrm{MBq} / \mathrm{m}^{3}$;

- une mesure continue du débit de rejet des effluents à la cheminée. Cette mesure est doublée, secourue électriquement et enregistrée en salle de commande.

Les contrôles périodiques, effectués sur quatre périodes mensuelles définies par la réglementation à savoir : 1er au 7, du 8 au 14, du 15 au 21, du 22 à la fin du mois, concernent:

- les principaux gaz rares sur un prélèvement instantané ;
- les aérosols prélevés sur filtre pour la mesure de l'activité bêta globale, la détermination des principaux radionucléides (PF et PA) et la mesure $\alpha$ globale d'origine artificielle (par une méthode garantissant un seuil de décision ne dépassant pas $\left.1 \times 10^{-3} \mathrm{~Bq} / \mathrm{m}^{3}\right)$;

- les halogènes (iodes) prélevés en continu pour l'analyse $\gamma$ globale et la détermination des iodes 131 et 133 ;

- le tritium gazeux prélevé en continu sur barboteur ;

- le carbone 14 capté en continu sur un tamis moléculaire analysé tous les trimestres.

\section{Effluents radioactifs gazeux issus du circuit} primaire hydrogéné sur l'EPR

Sur l'EPR, le traitement de ces effluents gazeux radioactifs issus du circuit primaire est différent de celui des autres centrales françaises. Ce circuit régule le taux d'oxygène et d'hydrogène des gaz au moyen d'un recombineur. Les gaz recombinés sont réutilisés dans les circuits ou orientés vers les " lits à retard » avant rejet à la cheminée. Les « lits à retard » permettent la décroissance radioactive des gaz rares (xénons, kryptons) et la rétention quasi-totale des iodes et des aérosols radioactifs (cf. figure 5). Ces rejets sont assimilés à des rejets permanents. 
Fig. 5 La conception et l'exploitation du circuit de collecte et de traitement des effluents gazeux hydrogénés du réacteur EPR sont différentes de celles des autres réacteurs français. Le schéma de principe ci-dessous illustre la collecte et le traitement des effluents issus du circuit primaire et de ses auxiliaires réalisés en boucle fermée ; les gaz en excès sont envoyés sur des lits à retard pour permettre leur décroissance radioactive avant contrôle et rejet.

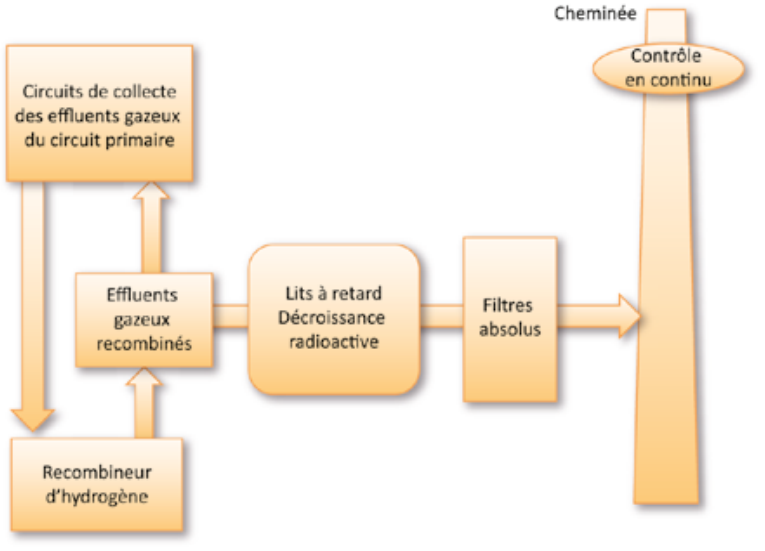

\section{Limites de rejet}

Pour les rejets radioactifs gazeux, la réglementation fixe (cf. encart p. 141) :

- une limite annuelle à ne pas dépasser pour chacune des cinq catégories de radionucléides réglementés. Celle-ci s'exprime en multiple de Bq (GBq ou TBq) ;

- des limites visant à assurer une bonne diffusion des rejets dans l'atmosphère en définissant:

- un débit d'activité à ne pas dépasser à la cheminée, exprimé en $\mathrm{Bq} / \mathrm{s}$,

- une activité volumique maximale pour l'air ambiant au niveau du sol, en $\mathrm{Bq} / \mathrm{m}^{3}$.

\section{Niveau des rejets d'effluents radioactifs} gazeux

La déclaration des rejets par catégories de radionucléides est établie sur la base des mesures réalisées dans le cadre des contrôles réglementaires et des règles de comptabilisation fixées par l'administration (cf. annexe 8.4).

Pour les cinq catégories de radionucléides réglementés, les rejets annuels moyens ramenés à un réacteur sont les suivants :

- les rejets de gaz rares (krypton, xénon, argon) varient généralement de $300 \mathrm{GBq}$ à $1000 \mathrm{GBq}$ environ (cf. figure 6). Les gaz rares étant inertes, ils ne sont pas retenus par les systèmes de filtration (filtres THE et pièges à iodes). Les valeurs plus élevées de 2003, 2007, 2008 et 2013, pouvant atteindre $2300 \mathrm{GBq}$, sont dues à des défauts d'étanchéité du gainage du combustible; les assemblages de combustible défectueux ont été remplacés ;
Fig. 6 L'activité moyenne des rejets annuels de gaz rares à la cheminée, rapportés à un réacteur, varie habituellement de 300 à $1000 \mathrm{GBq}$ environ, les valeurs les plus élevées étant dues à des défauts d'étanchéité du gainage du combustible. Les assemblages défectueux ont été remplacés.

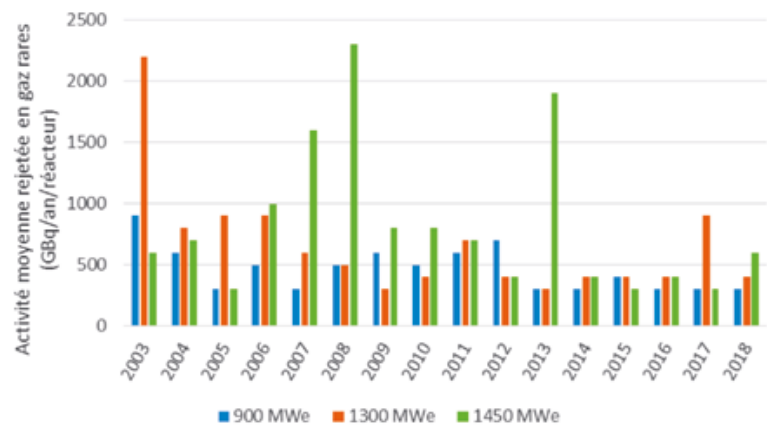

- le tritium gazeux est rejeté à $90 \%$ sous la forme de vapeur d'eau tritiée. L'activité des rejets est d'environ $350 \mathrm{GBq} /$ réacteur pour le palier de $900 \mathrm{MWe}$ et $650 \mathrm{GBq} /$ réacteur pour les paliers 1300 et 1450 MWe (cf. figure 7). Les rejets de tritium par voie atmosphérique sont issus de l'évaporation de l'eau tritiée de la piscine BK et de la piscine BR en arrêt de tranche. Sur le palier 1300 MWe, la ventilation des bâches TEP intermédiaires est un contributeur significatif aux rejets atmosphériques de tritium.

Fig. 7 L'activité moyenne des rejets annuels de tritium gazeux à la cheminée, rapportés à un réacteur, est d'environ $350 \mathrm{GBq}$ pour le palier $900 \mathrm{MWe}$ et $650 \mathrm{GBq}$ pour les paliers 1300 et 1450 MWe. Des modifications sur le prélèvement de ces rejets ont permis d'améliorer leur contrôle, entraînant une hausse des activités comptabilisées sans toutefois modifier les rejets réels. D'autres modifications sur le palier $1300 \mathrm{MWe}$ ont permis de diminuer les rejets dans l'environnement.

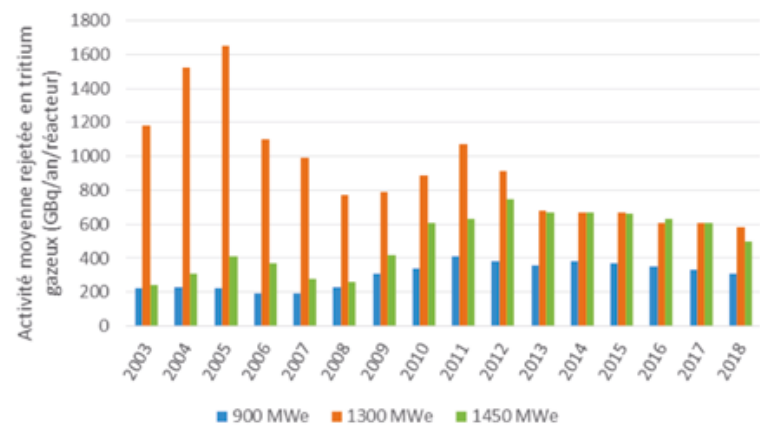

Des modifications relatives aux prélèvements du tritium à la cheminée ont été déployées sur l'ensemble des sites. Elles concernent:

- un prélèvement en continu avec des barboteurs réfrigérés (modification déployée entre 2005 et 2010), - un piquage à la cheminée du BAN (modification déployée entre 2008 et 2010). 


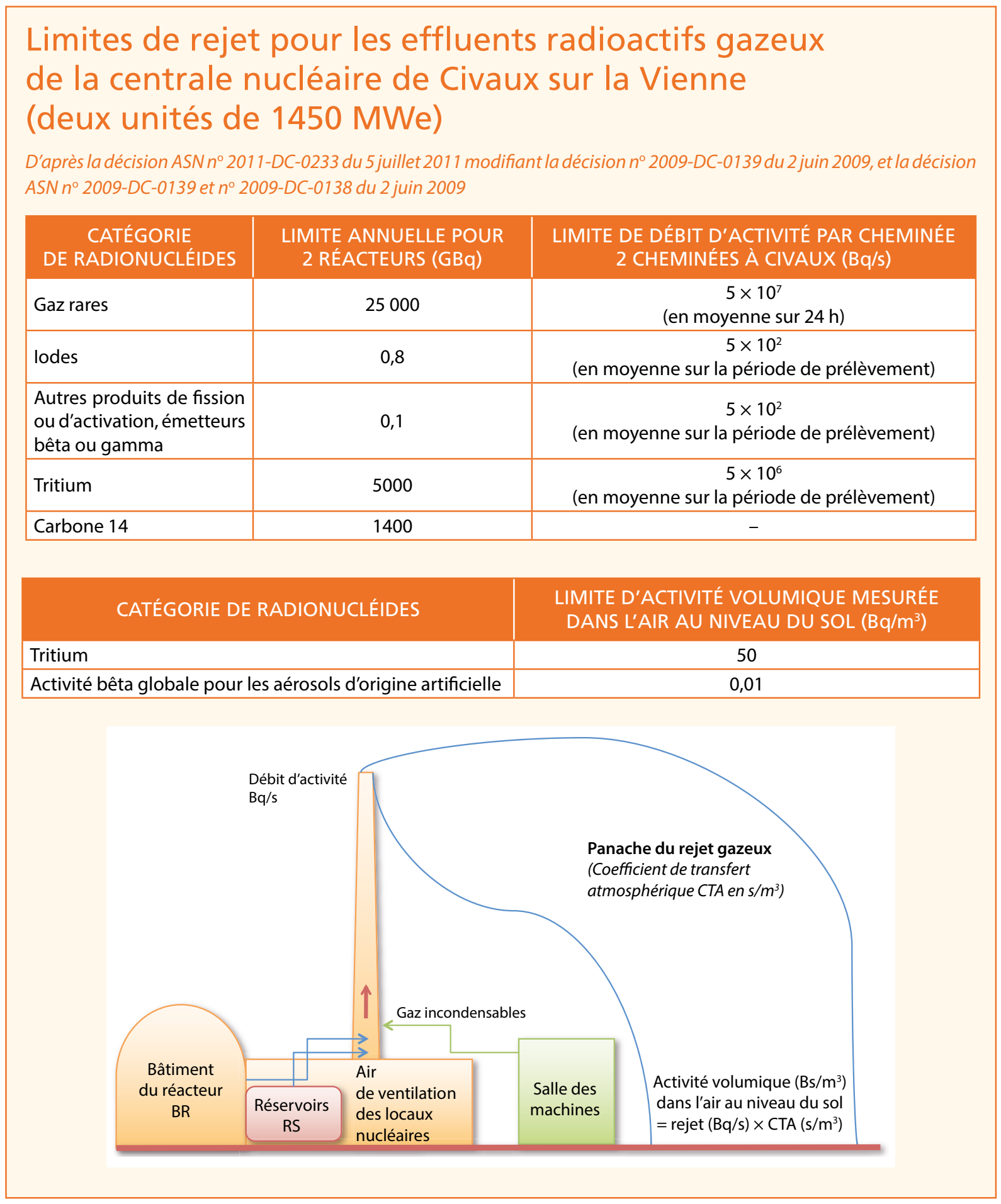

Ces modifications ont amélioré la représentativité et la qualité des prélèvements et entraîné une hausse des rejets comptabilisés sans toutefois modifier les rejets réels.

Enfin, une modification relative à la diminution du débit de ventilation des réservoirs de stockage des effluents primaires TEP a également été déployée sur le palier 1300 MWe (entre 2007 et 2009, la conception de ces réservoirs étant différente sur ce palier par rapport aux autres réacteurs du parc EDF), se traduisant par une baisse réelle des rejets ;

- le carbone $14^{1}$ est essentiellement rejeté à la cheminée sous la forme de méthane $\left(\mathrm{CH}_{4}\right)$ et de gaz carbonique $\left(\mathrm{CO}_{2}\right)$. Le carbone 14 est mesuré sur un prélèvement réalisé par un tamis moléculaire (cf. chapitre 11 sur la métrologie). Jusqu'en 2016, ces rejets pouvaient aussi être calculés à partir de l'énergie électrique brute produite à raison de

\footnotetext{
1. Avant 1999, le carbone 14 ne figurait pas dans l'inventaire des substances radioactives à comptabiliser. Depuis 1999, la réglementation demande de le comptabiliser. Cette exigence a été introduite à l'occasion de la publication des nouvelles décisions limites et modalités des centrales nucléaires.
} 
0,200 GBq/MWe.an'. Les rejets annuels d'activité de carbone 14 sont en moyenne compris entre $120 \mathrm{GBq}$ et $270 \mathrm{GBq}$ par unité de production ;

- les rejets d'iodes sont infimes (<0,04 GBq/an/réacteur en moyenne) compte tenu du bon confinement des circuits nucléaires et de l'efficacité des systèmes de piégeage ;

- les rejets de produits de fission (PF) et de produits d'activation (PA) émetteurs $\beta$ ou $\gamma$, présents sous forme de poussières (aérosols), sont, comme les rejets d'iodes, extrêmement faibles (0,004 GBq/ réacteur en moyenne).

\subsection{EFFLUENTS RADIOACTIFS LIQUIDES}

\section{Nature et origine}

Les effluents radioactifs liquides sont classés selon leur provenance. On distingue:

- les effluents provenant du circuit primaire dits " effluents primaires hydrogénés » contiennent des gaz de fission (xénons, iodes, césiums...), des produits d'activation (cobalts, manganèse, tritium, carbone 14...) mais aussi des substances chimiques telles que l'acide borique et le lithium et de l'hydrogène dissous (cf. \& 4). Ces effluents sont essentiellement produits en phase d'exploitation du fait des mouvements d'eau primaire effectués lors des variations de puissance ou de l'ajustement des paramètres chimiques de l'eau du réacteur ;

- les effluents issus des circuits auxiliaires dits « effluents usés » constituent le reste des effluents. Ils résultent principalement des opérations de maintenance (sur filtres, déminéraliseurs, échangeurs...) nécessitant des vidanges de circuit, des opérations d'évacuation du combustible usé et de conditionnement des résines usées, des actions de maintien de la propreté des installations (lavage du sol et du linge).

Nota: Les eaux issues des salles des machines (groupe turbo-alternateur) ne sont pas considérées comme des effluents radioactifs au sens de la réglementation dès lors que leurs activités volumiques n'excèdent pas $4000 \mathrm{~Bq} / \mathrm{L}$ en tritium et $4 \mathrm{~Bq} / \mathrm{L}$ en activité bêta globale (cf. § 3.4).

\section{Collecte et traitement}

Les effluents radioactifs liquides sont collectés de façon sélective avant d'être orientés vers les systèmes de traitement appropriés afin de retenir l'essentiel de leur radioactivité. Ils sont ensuite acheminés vers des réservoirs de stockage où ils subissent un contrôle, tant sur le plan radioactif que sur le plan chimique, avant d'être rejetés (cf. figure 8).

Fig. 8 Les effluents radioactifs liquides des centrales nucléaires sont classés en deux grandes catégories selon leur provenance : les effluents primaires hydrogénés (provenant du circuit primaire) et les effluents aérés usés (provenant des circuits auxiliaires). Ces effluents sont collectés, traités et contrôlés avant rejet, selon le schéma de principe ci-dessous.

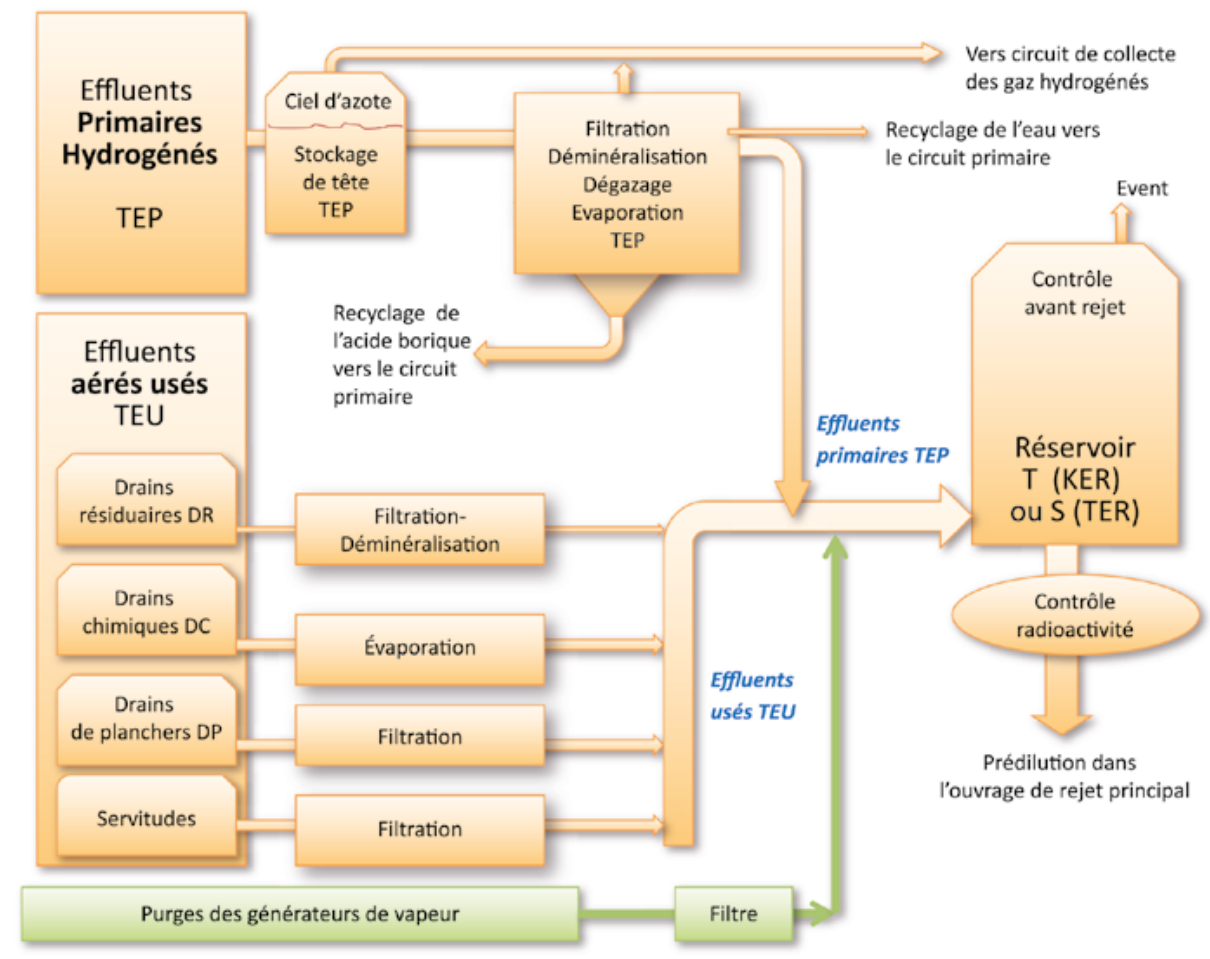

1. MWe.an : unité d'énergie correspondant à la production électrique d'une centrale de 1 MWe pendant un an. Ainsi, une centrale de 1000 MWe rejette : 0,200 GBq/MWe.an $\times 1000 \mathrm{MWe} \times 1$ an = $200 \mathrm{GBq}$ de carbone 14 


\section{Les effluents primaires hydrogénés}

Les effluents issus du circuit primaire sont dirigés vers le circuit de Traitement des Effluents Primaires (TEP) qui comporte :

- des réservoirs de collecte dits "réservoirs de tête » car situés en amont du traitement. Ces réservoirs sont maintenus sous atmosphère inerte d'azote en raison du dégazage de l'hydrogène contenu dans l'eau primaire. Si le réservoir contenait de l'air, donc de l'oxygène, il existerait un risque d'explosion lié au mélange " hydrogène-oxygène »;

- une chaîne de filtration et de déminéralisation visant à piéger les produits radioactifs, à l'exception du tritium, ainsi que les substances chimiques sauf l'acide borique ;

- un dégazeur ayant pour fonction d'extraire les gaz dissous (gaz rares radioactifs, hydrogène, azote) vers le circuit de collecte des effluents radioactifs gazeux;

- une chaîne d'évaporation permettant de séparer l'effluent traité en un distillat (eau) d'activité volumique faible pouvant être rejeté, voire recyclé le cas échéant, et un concentrat renfermant le bore qui est généralement recyclé vers le circuit primaire.

\section{Les effluents usés}

Les effluents liquides usés recueillis dans des puisards situés dans les locaux sont dirigés vers le circuit de traitement des effluents usés (TEU) où ils sont traités.

Collectés sélectivement suivant quatre catégories, le traitement de ces effluents est approprié à leurs caractéristiques physico-chimiques, ainsi :

- les drains résiduaires (DR) issus du circuit primaire ou de la piscine de désactivation du combustible usé sont traités par filtration pour retenir les matières en suspension et par déminéralisation (résines échangeuses d'ions) pour retenir les substances radioactives et chimiques en solution, sauf le tritium et l'acide borique ;

- les drains ou effluents chimiques (DC ou EC) passent sur une chaîne d'évaporation. On obtient alors, d'une part, un distillat épuré chimiquement et d'activité faible (quelques dizaines de $\mathrm{Bq} / \mathrm{L}$ à peine en activité bêta globale) et, d'autre part, un concentrat composé principalement d'acide borique jusqu'à des teneurs de $50 \mathrm{~g} / \mathrm{kg}$ en bore et de substances radioactives dont l'activité bêta/gamma peut varier généralement de $5 \mathrm{MBq} / \mathrm{L}$ à $15 \mathrm{MBq} / \mathrm{L}$. Pour éviter le risque de cristallisation de l'acide borique dans l'évaporateur, une quantité de soude est ajoutée à l'effluent afin de former du borate de sodium plus soluble que l'acide borique ;

- les drains de planchers (DP) qui sont filtrés;

- les effluents de servitudes (laverie du linge, douches...) peu radioactifs subissent une filtration avant d'être aiguillés vers les réservoirs $(T)$ de contrôle avant rejet.

Ces traitements permettent en outre de retenir les particules solides, de diamètre supérieur à $5 \mu \mathrm{m}$, en suspension dans les effluents avant leur entreposage dans les réservoirs dénommés réglementairement $(T)$, dits réservoirs de traitement.

Les réservoirs (S) dits « réservoirs de santé » doivent rester vides; ils ne peuvent être utilisés en secours des réservoirs $(T)$ qu'après accord préalable de l'ASN.

Dans ces réservoirs ( $T$ ) ou (S), les effluents sont analysés avant d'être rejetés dans les conditions fixées par la réglementation.

La capacité minimale d'entreposage $(T)$ et $(S)$ est fixée par la réglementation (ex. à Tricastin le volume minimal $(T)$ est fixé à $3000 \mathrm{~m}^{3}$ répartis en au moins 6 réservoirs de $500 \mathrm{~m}^{3}$; le volume (S) est de $1500 \mathrm{~m}^{3}$ répartis en au moins 3 réservoirs de $500 \mathrm{~m}^{3}$ ).

\section{Contrôle des rejets radioactifs liquides}

Tout effluent susceptible d'être radioactif ne peut être rejeté sans avoir été au préalable contrôlé dans les conditions fixées par la réglementation.

\section{Rejets radioactifs liquides issus des réservoirs T (KER) ou S (TER)}

\section{- Avant rejet}

Le rejet d'un réservoir T ou S ne peut être réalisé sans que les résultats d'analyse soient connus (tritium, activité alpha globale, activité bêta globale, activité gamma globale, composition isotopique par spectrométrie gamma). Si l'activité bêta globale excède $20 \mathrm{kBq} / \mathrm{L}$ (activités en tritium et potassium 40 exclues), l'effluent ne peut être rejeté et doit être retraité. Pour le carbone 14 et le nickel 63 dont les analyses sont longues et nécessitent des techniques de mesure complexes, il est admis de ne disposer des résultats qu'a posteriori. Ces effluents font aussi l'objet d'analyses chimiques (acide borique...).

\section{- Conditions de rejet}

Le débit de rejet doit être calculé pour permettre une pré-dilution minimale réglementaire dans les eaux de refroidissement avant le rejet dans le milieu aquatique.

Pour les sites implantés en bordure de cours d'eau, le rejet radioactif n'est autorisé que si le débit du cours d'eau est compris dans la plage fixée par la réglementation. En dehors de cette plage de débit (cas des étiages ou des crues), les rejets sont interdits. Toutefois, si les décisions limites et modalités de site le prévoient, le rejet peut être réalisé, à titre exceptionnel, après accord préalable de l'ASN et sous certaines conditions dûment précisées par l'ASN.

\section{- Pendant le rejet}

Une mesure de radioactivité est effectuée en continu sur la canalisation de rejet au moyen d'une chaîne de mesure $\gamma$ globale, doublée et secourue électriquement. En cas de dépassement d'un seuil d'alarme (40 kBq/L en activité $\gamma$ globale), le rejet est stoppé automatiquement. Pendant la durée du rejet, l'opérateur contrôle le débit de rejet et l'évolution du niveau 
du réservoir. Il ajuste, si nécessaire, le débit de rejet en fonction de celui du cours d'eau afin de respecter les limites de rejet fixées par la réglementation.

En période d'étiage, une coordination des rejets est mise en place entre les centrales situées sur un même bassin hydrographique (Loire-Vienne et Rhône) afin de minimiser les impacts des rejets (cf. chapitre 9 \& 5.3).

\section{- Contrôle du milieu récepteur}

Des contrôles (activité tritium et bêta globale) sont effectués à la station de prélèvement située en aval dans une zone de mélange. Les prélèvements des échantillons sont réalisés tous les jours pendant ou en dehors des périodes de rejet, à l'aide d'un hydrocollecteur. Des prélèvements sont aussi réalisés en amont du site en vue d'être éventuellement analysés en cas de problèmes (cf. annexe 8.3).

Pour les sites marins où la notion d'amont et d'aval n'existe pas, les contrôles sont effectués dans les puits ou bassins de rejets ou dans le canal de rejet (Gravelines) ainsi que dans la prise d'eau censée représenter l'amont (cf. annexe 8.4).

\section{Limites de rejet (réservoirs T ou S)}

Les contrôles effectués par l'exploitant visent à s'assurer du bon respect des limites fixées par la réglementation (cf. chapitre 6 sur le rôle de l'administration).

Les décisions limites et modalités fixent pour les rejets liquides :

- une limite annuelle à ne pas dépasser pour chacune des quatre catégories de radionucléides réglementés (tritium, carbone 14, iodes, autres PF ou PA). Celle-ci s'exprime en multiples de Bq (GBq ou TBq);

- des limites visant à assurer une bonne diffusion des rejets dans le milieu aquatique en définissant:

- un débit d'activité à ne pas dépasser, exprimé en $\mathrm{Bq} / \mathrm{s}$,

- une activité volumique maximale en aval des rejets, exprimée en $\mathrm{Bq} / \mathrm{L}$.

\section{Cas d'une centrale implantée sur un cours d'eau}

L'exemple des limites de rejets d'effluents radioactifs liquides de la centrale nucléaire de Dampierre est illustré par l'encart ci-contre.

\section{Cas d'une centrale implantée en bord de mer}

L'exemple des limites de rejets d'effluents radioactifs liquides de la centrale nucléaire de Penly est illustré par l'encart p. 145.

\section{Niveau annuel des rejets d'effluents radioac-} tifs liquides

Les rejets radioactifs liquides effectués par la vidange des réservoirs ( $T$ ) ou (S) représentent un volume annuel moyen par unité de production de $11000 \mathrm{~m}^{3}$ environ (cf. figure 9).

La déclaration des activités rejetées est établie, par catégorie de radionucléides, sur la base des mesures réalisées dans le cadre des contrôles réglementaires et des règles de comptabilisation fixées par l'admi-

\section{Limites de rejet pour les effluents radioactifs liquides de la centrale nucléaire de Dampierre sur la Loire (4 unités de 900 MWe)}

D'après les décisions ASN n 2011-DC-0210 et n²011-DC0211 du 3 mars 2011

\begin{tabular}{|c|c|c|}
\hline $\begin{array}{l}\text { CATÉGORIE } \\
\text { DE RADIONU- } \\
\text { CLÉIDES }\end{array}$ & $\begin{array}{c}\text { LIMITE } \\
\text { ANNUELLE } \\
\text { POUR } 4 \\
\text { RÉACTEURS } \\
\text { (GBq) }\end{array}$ & $\begin{array}{c}\text { LIMITE } \\
\text { DE DÉBIT } \\
\text { D'ACTIVITÉ } \\
\text { AU POINT } \\
\text { DE REJET } \\
(\mathrm{Bq} / \mathrm{s})\end{array}$ \\
\hline Tritium & 100000 & $80 \times D^{*}$ \\
\hline Carbone 14 & 260 & - \\
\hline lodes & 0,6 & $0,1 \times D^{*}$ \\
\hline $\begin{array}{l}\text { Autres produits de } \\
\text { fission ou d'activa- } \\
\text { tion émetteurs bêta } \\
\text { ou gamma }\end{array}$ & 36 & $0,7 \times D^{*}$ \\
\hline
\end{tabular}

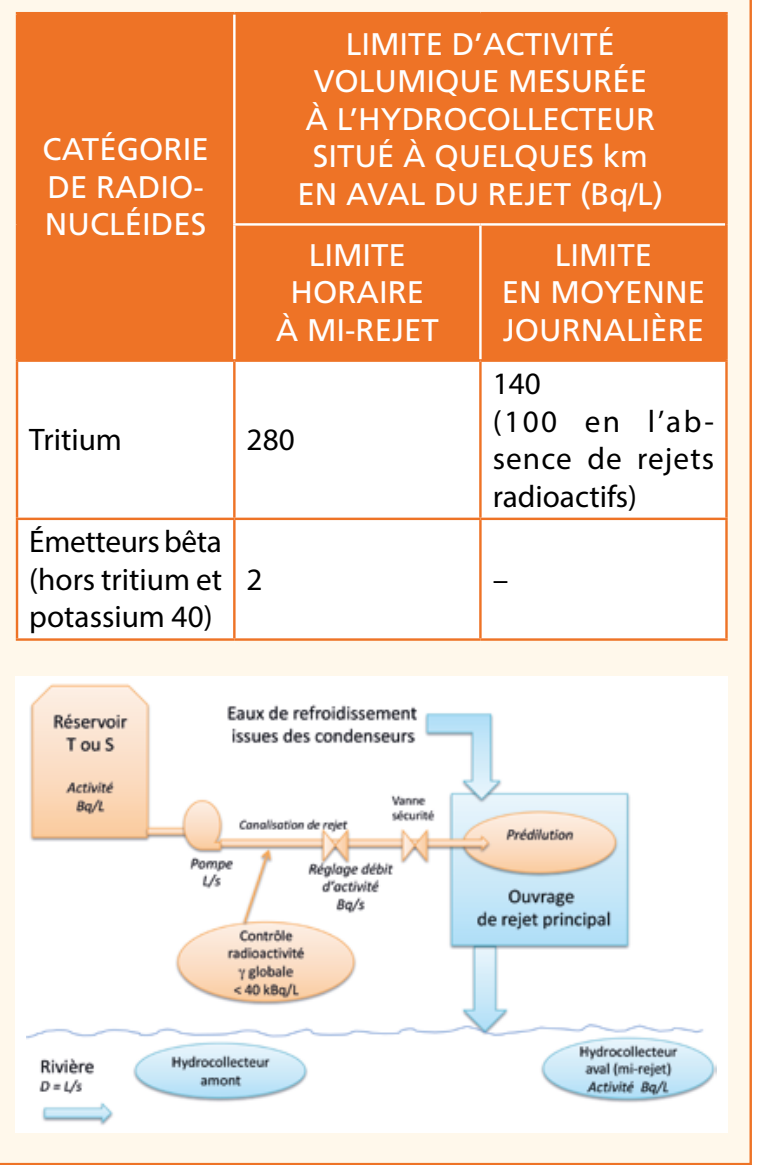

nistration (cf. annexe 8.5). Pour les quatre catégories de radionucléides réglementés, le niveau des rejets annuels est le suivant:

- le tritium dans l'eau du circuit primaire provient essentiellement de l'activation du bore 10 et du lithium 6. Par ailleurs, les réacteurs des paliers 


\section{Limites de rejet pour les effluents radioactifs liquides de la centrale nucléaire de Penly sur la Manche (2 unités de $1300 \mathrm{MWe})$}

D'après l'arrêté d'autorisation du 15 février 2008 et la décision ASN n²008-DC-0090 du 10 janvier 2008

\begin{tabular}{|c|c|c|}
\hline $\begin{array}{l}\text { CATÉGORIE } \\
\text { DE RADIO- } \\
\text { NUCLÉIDES }\end{array}$ & $\begin{array}{c}\text { LIMITE } \\
\text { ANNUELLE } \\
\text { POUR } \\
2 \text { RÉACTEURS } \\
(\mathrm{GBq})\end{array}$ & $\begin{array}{l}\text { LIMITE DE } \\
\text { DÉBIT } \\
\text { D'ACTIVITÉ } \\
\text { AU POINT } \\
\text { DE REJET } \\
\text { EN MER } \\
(\mathrm{Bq} / \mathrm{s})\end{array}$ \\
\hline Tritium & $80000 *$ & $800 \times D^{* *}$ \\
\hline Carbone 14 & 190 & - \\
\hline lodes & 0,1 & $1 \times D^{* *}$ \\
\hline $\begin{array}{l}\text { Autres pro- } \\
\text { duits de fis- } \\
\text { sion ou d'acti- } \\
\text { vation, émet- } \\
\text { teurs bêta ou } \\
\text { gamma }\end{array}$ & 25 & $7 \times D^{* *}$ \\
\hline
\end{tabular}

\begin{tabular}{|c|c|c|}
\hline \multirow{2}{*}{$\begin{array}{l}\text { CATÉGORIE } \\
\text { DE RADIO- } \\
\text { NUCLÉIDES }\end{array}$} & \multicolumn{2}{|c|}{$\begin{array}{c}\text { LIMITE D'ACTIVITÉ } \\
\text { VOLUMIQUE MESURÉE } \\
\text { À L'HYDROCOLLECTEUR } \\
\text { SITUÉ DANS LE PUITS } \\
\text { OU BASSIN DE REJET (Bq/L) }\end{array}$} \\
\hline & $\begin{array}{c}\text { LIMITE } \\
\text { HORAIRE À } \\
\text { MI-REJET }\end{array}$ & $\begin{array}{c}\text { LIMITE } \\
\text { EN MOYENNE } \\
\text { JOURNALIĖRE }\end{array}$ \\
\hline Tritium & 1800 & $\begin{array}{l}900 \\
(100 \text { en l'ab- } \\
\text { sence de rejets } \\
\text { radioactifs) }\end{array}$ \\
\hline $\begin{array}{l}\text { Émetteurs bêta } \\
\text { hors tritium et } \\
\text { potassium } 40\end{array}$ & 18 & - \\
\hline
\end{tabular}

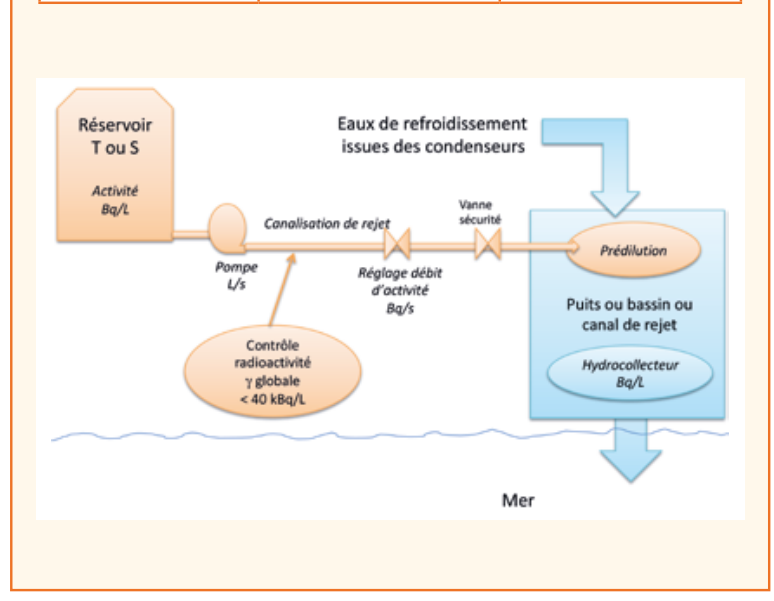

Fig. 9 Le volume annuel moyen d'effluents radioactifs rejeté par les réservoirs $\mathrm{T}$ ou $\mathrm{S}$, rapporté à un réacteur, est d'environ $11000 \mathrm{~m}^{3}$.

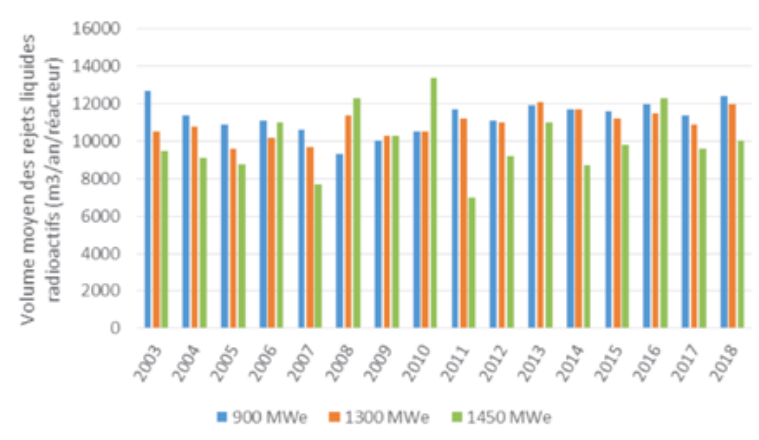

1300 MWe et 1450 MWe comportent des grappes sources secondaires de neutrons à l'origine d'une production supplémentaire de tritium comprise entre 4 et 12 TBq par an et par réacteur.

Le tritium du circuit primaire se retrouve dans les effluents sous forme d'eau tritiée ; du fait de son niveau de concentration très faible, il n'existe pas de procédé industriel permettant son élimination, contrairement à d'autres radionucléides. Étant très peu radiotoxique (émetteur bêta de faible énergie), le tritium est rejeté dans l'environnement.

Les rejets sont en moyenne par réacteur d'environ $10000 \mathrm{GBq}(10 \mathrm{TBq})$ à $35000 \mathrm{GBq}(35 \mathrm{TBq})$, selon le palier de puissance du réacteur, le mode de gestion de combustible et la présence ou non de grappes sources secondaires de neutrons (cf. figure 10) ;

Fig. $10 \triangleright$ Du fait du niveau de concentration très faible du tritium dans l'eau du circuit primaire, il n'existe pas de procédé industriel permettant une élimination de ce radionucléide. L'activité moyenne des rejets annuels de tritium liquides, rapportés à un réacteur, est de l'ordre de 10 à 35 terabecquerels selon la puissance du réacteur, le mode de gestion du combustible et la présence ou non de grappes sources secondaires de neutrons.

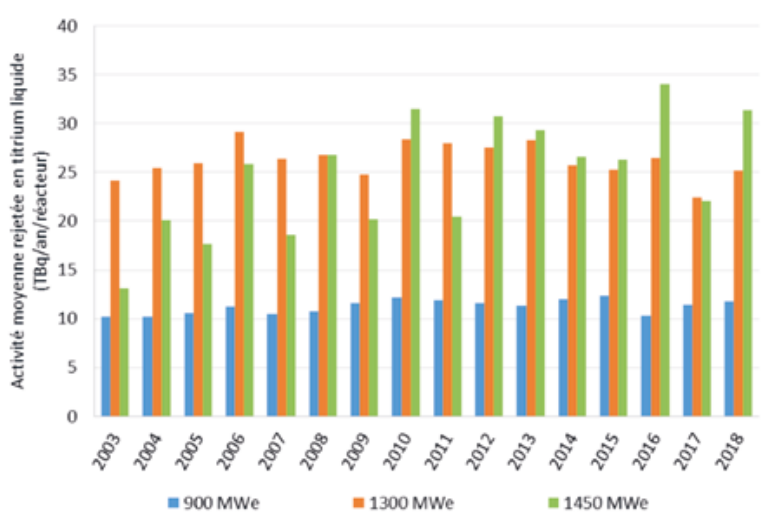

- les rejets de carbone 14, présent dans l'effluent sous forme de $\mathrm{CO}_{2}$ dissous, représentent entre 8 
et 20 GBq par réacteur, soit environ $10 \%$ des rejets de carbone 14 par voie atmosphérique ;

- les rejets d'iodes (PF) sont très faibles, inférieurs à 0,01 GBq par réacteur. Ceci s'explique par plusieurs raisons :

- les iodes formés dans le combustible restent confinés en quasi-totalité au sein du combustible grâce au gainage. En cas d'inétanchéité de ce dernier, les iodes et d'autres produits de fission peuvent migrer dans l'eau primaire. La radioactivité de l'eau du circuit primaire, et particulièrement celles des iodes, est suivie et ne peut dépasser les niveaux fixés par les spécifications radiochimiques. Le circuit primaire est équipé d'un circuit de purification en continu (circuit RCV) qui maintient le niveau de radioactivité de l'eau primaire le plus bas possible,

- les iodes de l'eau issue du circuit primaire sont piégés efficacement par les systèmes de traitement d'effluents,

- les iodes 131 et 133 ont des périodes radioactives courtes (respectivement 8 jours et 21 heures) et disparaissent rapidement par décroissance radioactive.

- les produits de fission (PF) et d'activation (PA) sont éliminés en grande partie par les systèmes de traitement. Les rejets d'activité de ces radionucléides par unité de production ont diminué d'un facteur 100 depuis la mise en service du parc nucléaire d'EDF. Les améliorations apportées aux circuits de collecte et de traitement et les efforts réalisés par l'exploitant pour réduire à la source la production d'effluents expliquent ce résultat (cf. figure 11). Les cobalts 58 et 60, l'argent $110 \mathrm{~m}$

Fig. 11 Les produits de fission et d'activation présents dans l'eau des circuits de l'îlot nucléaire sont éliminés en grande partie par les systèmes de traitement des effluents. Les efforts réalisés pour réduire ces effluents à la source et les améliorations apportées à leur collecte et leur traitement ont permis de réduire d'un facteur 100 (depuis la mise en service du parc nucléaire EDF) l'activité moyenne des rejets annuels de produits de fission et d'activation, rapportés à un réacteur. Aujourd'hui, cette activité représente moins de 0,5 gigabequerel par an et par réacteur.

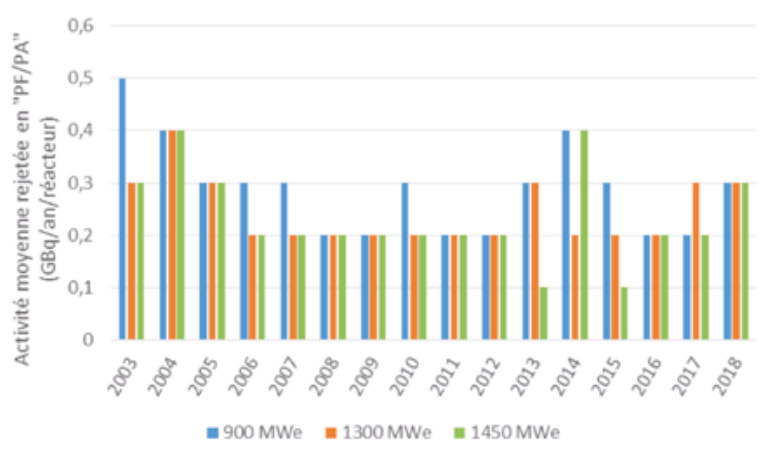

et le nickel 63 sont prépondérants dans ces rejets qui représentent en moyenne moins de 0,5 GBq par réacteur (cf. chapitre 9 sur la maîtrise des impacts des rejets).

\subsection{EAUX D'EXHAURE DES SALLES DES MACHINES}

\section{Nature et collecte}

Les eaux issues du circuit secondaire (non radioactif) et les eaux des puisards des salles des machines contiennent des substances chimiques utilisées pour le conditionnement chimique du circuit secondaire contre la corrosion, à savoir : la morpholine, l'éthanolamine, l'ammoniaque, l'hydrazine notamment (cf. § 4).

Ces eaux sont collectées sans traitement préalable vers des réservoirs dénommés Ex (appellation réglementaire, Ex signifiant « exhaure ») où elles sont contrôlées avant d'être rejetées. La capacité minimale d'entreposage Ex est fixée par la réglementation. À titre d'exemple, la capacité est fixée à $2000 \mathrm{~m}^{3}$ répartis en au moins deux réservoirs de $1000 \mathrm{~m}^{3}$ à la centrale de Tricastin.

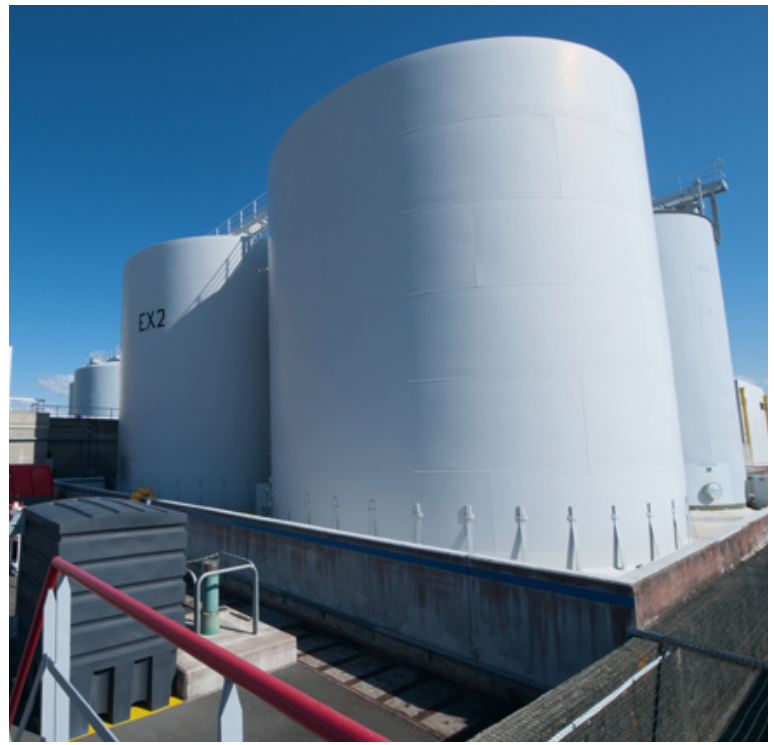

Réservoirs Ex et leurs cuvelages de rétention : centrale du Blayais (c) EDF - Gabrielle Balloffet

Les eaux issues des salles des machines ne sont pas classées dans les effluents radioactifs. Elles peuvent toutefois présenter des traces de radioactivité lorsque les tubes des générateurs de vapeur (qui constituent la deuxième barrière entre l'eau radioactive du circuit primaire et l'eau du circuit secondaire) ne sont pas parfaitement étanches. Dans ce cas, des radionucléides, comme le tritium, peuvent migrer du circuit primaire vers le circuit secondaire et contaminer les eaux d'exhaure qui ne pourront pas être rejetées et devront être traitées comme un effluent radioactif. Le volume annuel moyen d'eau d'exhaure rejeté est d'environ $55000 \mathrm{~m}^{3}$ pour une unité de production (cf. figure 12). 
Fig. 12 Le volume annuel moyen d'eaux d'exhaure rejeté par les réservoirs Ex, rapporté à un réacteur, est d'environ $55000 \mathrm{~m}^{3}$.

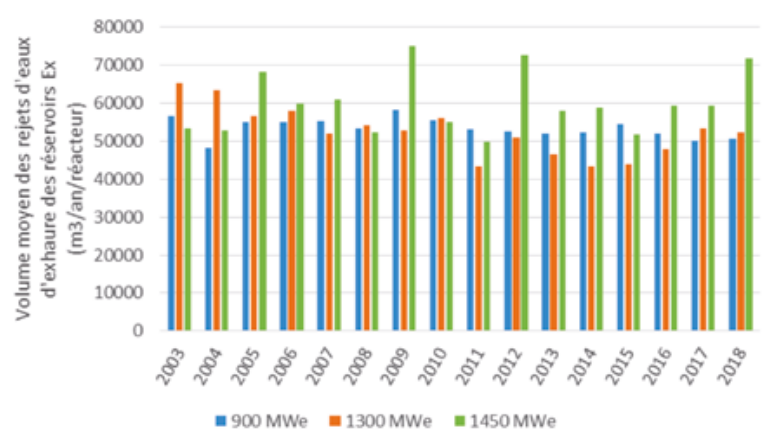

Limites relatives à la radioactivité des eaux issues des salles des machines (Ex)

Les valeurs de radioactivité, au-delà desquelles les eaux d'exhaure des salles des machines sont considérées comme des effluents radioactifs, sont indiquées dans les décisions limites et modalités des sites (cf. tableau I).

Tableau I Les eaux issues de la salle des machines ne sont pas classées dans les effluents radioactifs mais peuvent présenter des traces de radioactivité lorsque les tubes des générateurs de vapeur ne sont pas parfaitement étanches. Les valeurs de radioactivité au-delà desquelles ces eaux doivent être traitées et rejetées dans les mêmes conditions qu'un effluent radioactif sont réglementaires; le tableau ci-dessous en donne un exemple.

\begin{tabular}{|c|c|}
\hline $\begin{array}{l}\text { CATÉGORIE DE } \\
\text { RADIONUCLÉIDES }\end{array}$ & $\begin{array}{c}\text { LIMITE D'ACTIVITÉ } \\
\text { VOLUMIQUE DANS } \\
\text { LE RÉSERVOIR } \\
\text { Bq/L }\end{array}$ \\
\hline Tritium & 4000 \\
\hline $\begin{array}{l}\text { Activité bêta glo- } \\
\text { bale (hors tritium et } \\
\text { potassium 40) }\end{array}$ & 4 \\
\hline
\end{tabular}

Si l'activité en tritium est comprise entre $400 \mathrm{~Bq} / \mathrm{L}$ et $4000 \mathrm{~Bq} / \mathrm{L}$, le rejet tritium par cette voie doit être pris en compte dans le calcul du débit d'activité $(\mathrm{Bq} / \mathrm{s})$ exigé pour les rejets radioactifs liquides. Au-dessus de $4000 \mathrm{~Bq} / \mathrm{L}$ en tritium ou $4 \mathrm{~Bq} / \mathrm{L}$ en activité bêta globale, ces eaux sont rejetées dans les mêmes conditions qu'un effluent radioactif, après traitement éventuel, dans le cadre de dispositions particulières de rejet après accord préalable de l'ASN.

\subsection{REJETS GAZEUX DIFFUS}

Les rejets radioactifs diffus ont notamment pour origine :

- Les évents de réservoirs d'entreposage des effluents radioactifs ( $T, S$ et $E x$ ), le réservoir de stockage de l'eau borée pour le remplissage des piscines ;
- les rejets de vapeur du circuit secondaire par le système de décharge à l'atmosphère, susceptibles de renfermer de la radioactivité en cas d'inétanchéité des tubes de générateurs de vapeur.

Ces rejets, ne transitant pas par la cheminée instrumentée, font l'objet d'une estimation mensuelle par calcul visant notamment à s'assurer qu'ils sont conformes aux hypothèses retenues dans l'étude d'impact de l'installation. Les résultats de ces estimations, qui portent en particulier sur les volumes et activités rejetés (tritium, iodes...), sont mentionnés dans les registres réglementaires communiqués mensuellement à l'administration.

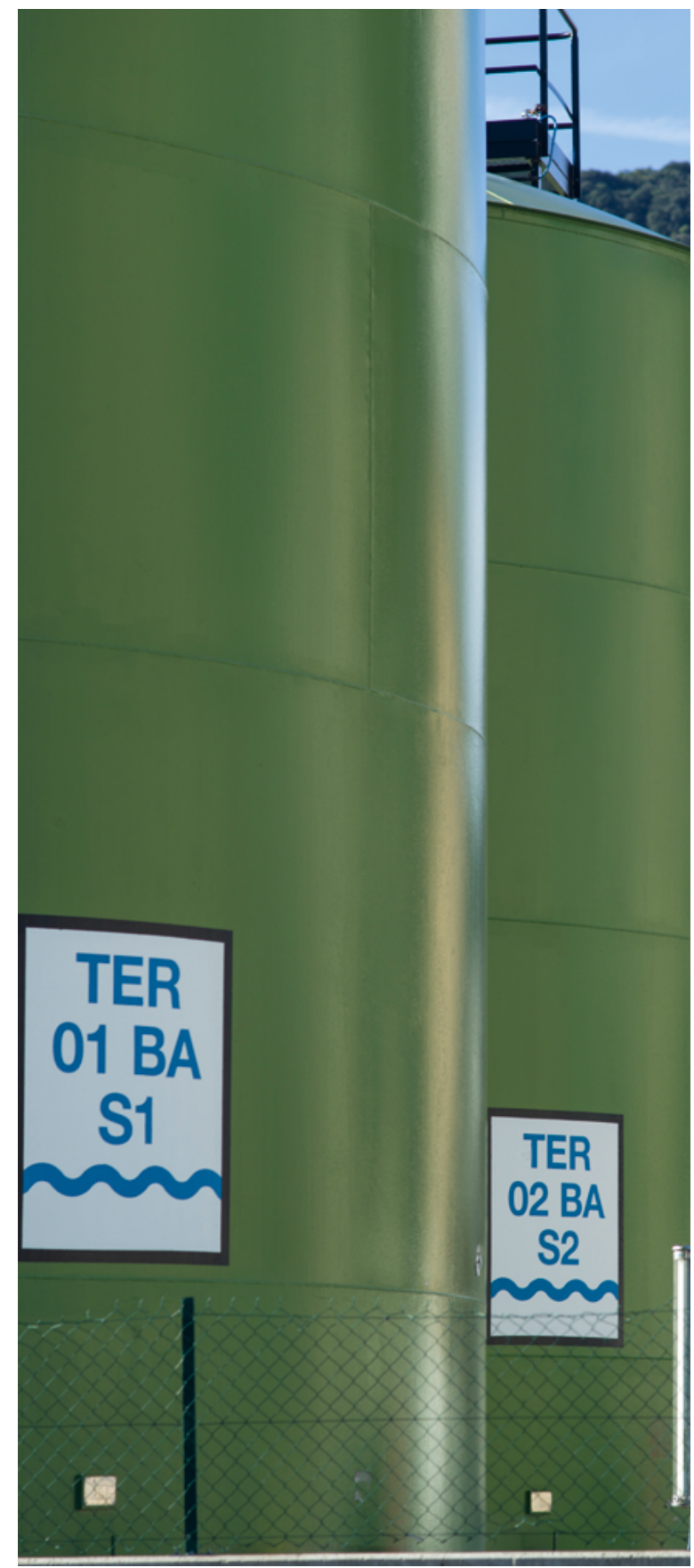

Réservoirs T d'entreposage des effluents radioactifs avant rejet : centrale de Cruas - (c) EDF - Christel Sasso 


\section{Domaine chimique}

\subsection{ORIGINE DES SUBSTANCES CHIMIQUES REJETÉES}

Le fonctionnement d'une centrale nucléaire nécessite l'utilisation de substances chimiques et donne lieu à des rejets chimiques par voie liquide dans l'environnement et, dans une moindre mesure, à des rejets gazeux non radioactifs.

Chaque substance chimique assure une fonction bien précise tant en phase d'exploitation (contrôle de la réaction nucléaire, protection des circuits contre la corrosion, lutte contre le tartre et le développement de micro-organismes, production d'eau déminéralisée...), que lors des opérations de maintenance (lessivage chimique...).

La présence d'impuretés dans les produits chimiques commerciaux et l'usure des tubes en laiton des condenseurs (pour les centrales qui en sont équipées) conduisent à des rejets de métaux.

\section{Substances utilisées pour l'exploitation}

\section{Contrôle de la réaction nucléaire}

L'acide borique $\left(\mathrm{H}_{3} \mathrm{BO}_{3}\right)$, en solution dans l'eau du circuit primaire, participe, avec les grappes de commande, au contrôle de la réaction nucléaire. Seul le bore 10, isotope présent à hauteur de $20 \%$ environ dans le bore naturel, permet d'absorber les neutrons en formant du tritium (radioactif) ou du lithium 7 (stable). C'est un acide faible qui se retrouve dans les effluents radioactifs lorsqu'il est déchargé du circuit primaire. La concentration en bore dans l'eau du circuit primaire varie au cours du cycle de fonctionnement du réacteur'. La teneur en bore en début de cycle est déterminée en fonction de paramètres neutroniques et de la longueur de cycle souhaitée. Sa concentration est ensuite progressivement abaissée, au cours du cycle, pour compenser l'épuisement du combustible ; elle évolue, à titre d'exemple, d'environ 1200 à 1800 mg/kg (ppm) en début de cycle à presque $0 \mathrm{mg} / \mathrm{kg}$ (ppm) en fin de cycle (cf. figure 13).

\section{Conditionnement chimique du circuit primaire}

La lithine ( $\mathrm{LiOH})$ est utilisée en faible quantité (quelques dizaines de kilos par an et par réacteur) pour assurer un $\mathrm{pH}$ de moindre corrosion des structures métalliques du circuit primaire. La lithine est enrichie en lithium 7 (> 99,9\%) pour éviter la formation de tritium par activation du lithium 6 présent dans le lithium naturel.
Fig. 13 Le bore est utilisé dans le circuit primaire pour contrôler la réaction nucléaire. La concentration en bore est d'environ 1200 à $1800 \mathrm{mg} / \mathrm{kg}$ en début de cycle; elle est abaissée régulièrement au cours du cycle, en fonction de l'épuisement du combustible, pour atteindre presque $0 \mathrm{mg} / \mathrm{kg}$ en fin de cycle.

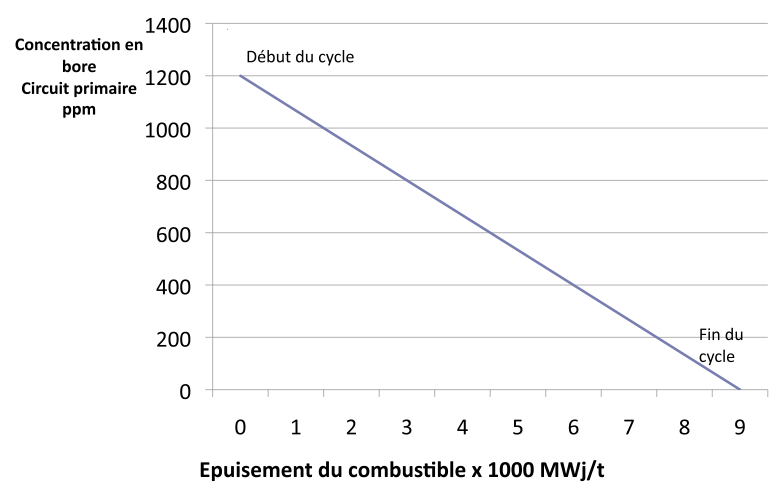

L'hydrazine $\left(\mathrm{N}_{2} \mathrm{H}_{4}\right)$ est injectée, sous forme d'hydrate d'hydrazine $\left(\mathrm{N}_{2} \mathrm{H}_{4}, \mathrm{H}_{2} \mathrm{O}\right)$, pendant la phase de démarrage du réacteur (palier chimique) afin d'éliminer l'oxygène dissous dans l'eau du circuit primaire.

\section{Conditionnement chimique du circuit secondaire (eau-vapeur) en fonctionnement}

Le conditionnement chimique du circuit secondaire vise à éviter la corrosion des matériels par l'utilisation de substances chimiques choisies pour leur efficacité mais aussi pour leur moindre impact sur la santé et l'environnement.

L'hydrazine $\left(\mathrm{N}_{2} \mathrm{H}_{4}\right)$ est une base faible utilisée en fonctionnement et pour la conservation en eau des matériels lors des arrêts de tranche. Elle permet de minimiser la corrosion des matériaux en réagissant avec l'oxygène dissous dans l'eau. Injectée sous forme d'hydrate d'hydrazine $\left(\mathrm{N}_{2} \mathrm{H}_{4}, \mathrm{H}_{2} \mathrm{O}\right)$, l'hydrazine se décompose thermiquement en ammoniaque $\left(\mathrm{NH}_{4} \mathrm{OH}\right)$ qui joue un rôle sur le $\mathrm{pH}$ de l'eau du circuit secondaire. L'hydrazine agit donc tant sur l'oxygène dissous que sur le $\mathrm{pH}$ de l'eau du circuit. L'hydrazine est une substance classée CMR (cancérogène, mutagène et repro-toxique) ; toutefois, dans l'état actuel des connaissances, il n'existe pas de substitut efficace à l'hydrazine pour le conditionnement chimique du circuit secondaire des centrales REP.

La morpholine $\left(\mathrm{C}_{4} \mathrm{H}_{9} \mathrm{NO}\right)$, l'éthanolamine $\left(\mathrm{C}_{2} \mathrm{H}_{7} \mathrm{NO}\right)$ sont des amines volatiles. Ces amines et l'ammoniaque $\left(\mathrm{NH}_{4} \mathrm{OH}\right)$ peuvent être employées, seules ou en combinaison, pour maintenir un $\mathrm{pH}$ de moindre 


\section{Évolution du mode de conditionnement chimique du circuit secondaire}

Jusqu'au milieu des années 1980, pour prévenir les risques de corrosion, l'eau du circuit secondaire des centrales nucléaires REP d'EDF était conditionnée à l'ammoniaque. Cette substance avait été retenue pour différentes raisons (mise en œuvre facile, produit courant bénéficiant d'un bon retour d'expérience, absence de formation de produits de décomposition, faible coût). Pour éviter la corrosion ammoniacale des matériels renfermant du cuivre (tubes en laiton des condenseurs et des réchauffeurs basse pression), le $\mathrm{pH}$ dans l'eau alimentaire des générateurs de vapeur des unités concernées était volontairement limité à 9,2 à $25^{\circ} \mathrm{C}$ (mode « bas $\left.\mathrm{pH} »\right)$.

À partir des années 1983-1984, l'ammoniaque a été remplacée par la morpholine sur les unités « à alliages cuivreux » pour traiter plus efficacement le phénomène de corrosion-érosion rencontré sur les structures en acier au carbone.

Sur les autres unités, sans cuivre, le pH a été remonté entre 9,6 et 9,8 (mode « haut $\mathrm{pH}$ ») pour mieux maîtriser la corrosion généralisée, notamment celle des aciers au carbone, et réduire la quantité de produits de corrosion véhiculés dans les circuits (générateurs de vapeur...). Ammoniaque ou morpholine pouvaient être utilisées indifféremment avec toutefois une préférence pour la morpholine, plus efficace pour diminuer les produits de corrosion véhiculés dans les circuits.

En 2010, sur les 58 unités en exploitation, 50 utilisent la morpholine, les autres l'ammoniaque. II convient aussi de noter que certaines unités fonctionnant à haut $\mathrm{pH}$ à la morpholine utilisent, en complément, de l'ammoniaque (cas de Gravelines).

Depuis 2011, la morpholine est progressivement remplacée par l'éthanolamine, dont les caractéristiques sont favorables à une meilleure protection des aciers non ou faiblement alliés contre les phénomènes de corrosion-érosion. L'éthanolamine étant par ailleurs une base plus forte que la morpholine et l'ammoniaque, l'obtention du pH requis dans l'eau d'alimentation des générateurs de vapeur nécessite des concentrations molaires plus faibles. Outre sa moindre toxicité, l'éthanolamine présente une meilleure stabilité thermique que la morpholine.

En 2020, la majorité des centrales françaises est conditionnée à l'éthanolamine. Le conditionnement chimique cible du circuit secondaire est de $4 \mathrm{mg} / \mathrm{L}$ en éthanolamine et $1,5 \mathrm{mg} / \mathrm{L}$ en ammoniaque dans l'eau alimentaire des générateurs de vapeur, afin de maîtriser également les risques de colmatage des GV.

corrosion dans le circuit secondaire. Elles complètent l'action de l'hydrazine. Le conditionnement chimique du circuit secondaire fait l'objet de spécifications chimiques. Celles-ci sont établies en tenant compte notamment de la nature des matériaux à protéger contre la corrosion (aciers au carbone, aciers inoxydables, alliages cuivreux) mais aussi des questions de santé et d'environnement, car ces substances et leurs dérivés se retrouvent en partie dans les rejets. Le mode de conditionnement chimique du circuit secondaire a évolué avec les années pour tenir compte du retour d'expérience interne et étranger (cf. encart ci-contre).

L'éthanolamine (ETA) est désormais utilisée sur la majorité des centrales françaises, en remplacement de la morpholine, afin de limiter les phénomènes de corrosion-érosion en tout point du poste d'eau et d'obtenir le meilleur compromis entre la protection des matériels et les rejets. En effet, grâce à sa plus forte basicité, les quantités nécessaires d'ETA sont plus faibles que celles de morpholine pour maintenir le $\mathrm{pH}$ de moindre corrosion ; l'ETA génère donc moins de rejets. Par ailleurs, l'ETA est plus biodégradable que la morpholine.

\section{Conditionnement chimique des circuits de refroidisse- ment intermédiaires}

Le phosphate trisodique $\left(\mathrm{Na}_{3} \mathrm{PO}_{4}\right)$ est utilisé pour le conditionnement chimique des circuits de refroidissement intermédiaires (circuits RRI, SRI, TRI). Ces circuits sont dits « intermédiaires » car ils assurent l'échange de chaleur entre un circuit radioactif (circuit primaire par exemple) et un circuit d'eau brute ouvert sur l'environnement. Le conditionnement chimique de ces circuits doit permettre de minimiser la corrosion des matériaux et les appoints-rejets à mettre en œuvre pour conserver une chimie correcte. En cas d'inétanchéité des échangeurs, ce circuit intermédiaire peut être contaminé en particulier par le tritium. Lorsque l'activité du circuit dépasse la limite des spécifications radiochimiques, des investigations sont à engager sur le circuit afin de localiser une éventuelle inétanchéité. L'eau du circuit peut également être le siège d'une carbonatation du fait de la présence de gaz carbonique dans l'air du réservoir tampon. Dans les deux cas, une vidange du circuit peut être nécessaire, produisant des effluents phosphatés (cf. figure 14).

\section{Lavage du linge contaminé (laverie)}

Le lavage du linge utilisé par le personnel intervenant en zone contrôlée fait appel à des lessives commerciales. Les fabricants de lessive sont tenus, par le cahier des charges, de fournir des lessives biodégradables, exemptes de phosphates et de produits chélatants tels que l'EDTA (acide éthylènediaminetétraacétique) et le NTA (acide nitrilotriacétique). Les effluents issus du lavage du linge sont filtrés ce qui permet de retenir la radioactivité mais pas les détergents. Ces effluents ainsi traités sont ensuite aiguillés vers les réservoirs $T$ de contrôle avant rejet. Les quantités de détergents rejetés varient en fonction du volume de linge lavé. Ainsi, l'ordre de grandeur des volumes d'effluents de laverie varie de quelques dizaines de $\mathrm{m}^{3}$ par an et par réacteur à environ 
Fig. 14 Principe d'un circuit intermédiaire assurant l'échange de chaleur entre un circuit nucléaire radioactif et un circuit d'eau brute ouvert sur l'environnement. Pour minimiser la corrosion de leurs matériaux, ces circuits sont conditionnés au phosphate.

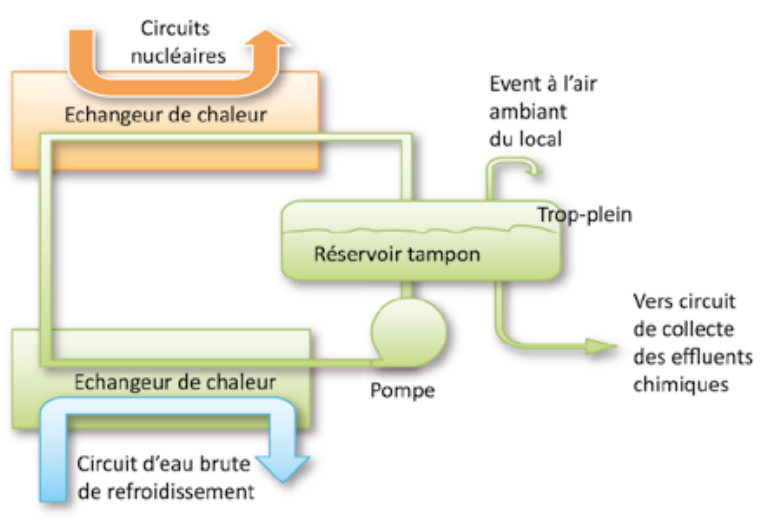

2000 m³/an/réacteur. Les quantités de détergents rejetés sont en moyenne d'une dizaine de kg par an et par réacteur ; elles peuvent atteindre une centaine de kilos par an en pointe, ramenées à un réacteur. Les sites s'engagent dans la maitrise de la propreté radiologique via le basculement en mode EVEREST (Évoluer VERs une Entrée Sans Tenue universelle). Le mode EVEREST implique l'entrée en bleu de travail dans les zones propres puis en tenue adaptée dans les zones contaminées. Une des conséquences du basculement est une baisse de la quantité de tenues lavées et donc des quantités de détergents rejetées.

Traitement contre le tartre des circuits dits «fermés » de refroidissement des condenseurs

L'acide sulfurique $\left(\mathrm{H}_{2} \mathrm{SO}_{4}\right)$ est utilisé pour prévenir la formation du tartre. L'injection d'acide permet de baisser le $\mathrm{pH}$ de l'eau du circuit de refroidissement et ainsi de déplacer l'équilibre des ions hydrogénocarbonates, empêchant la formation de tartre :

$$
\mathrm{H}^{+}+\mathrm{HCO}_{3}^{-} \rightarrow \overrightarrow{\mathrm{CO}_{2}}+\mathrm{H}_{2} \mathrm{O}
$$

Les sulfates, associés à l'utilisation d'acide sulfurique, se retrouvent dans la purge du circuit et donc dans l'ouvrage de rejet principal.

Les antitartres organiques peuvent également être utilisés pour lutter contre l'encrassement des aéroréfrigérants, en complément de l'acide sulfurique. Ils se retrouvent alors en partie dans l'ouvrage de rejet. L'injection de ces substances est ajustée en fonction de la qualité d'eau à traiter et des contraintes fixées sur les rejets (cf. tableau II).

Traitement biocide des circuits dits «fermés » de refroidissement des condenseurs

La monochloramine utilisée pour le traitement biocide est produite in situ en mélangeant de l'eau de
Javel $(\mathrm{NaClO})$ à de l'ammoniaque $\left(\mathrm{NH}_{4} \mathrm{OH}\right)$ dans de l'eau utilisée comme fluide moteur. Celle-ci est directement injectée dans le circuit d'eau de refroidissement. Elle réagit, au cours du traitement, pour former différentes substances chimiques qui se retrouvent dans les purges de l'aéroréfrigérant et donc dans l'ouvrage de rejet principal (cf. figure 15). Il s'agit principalement de chlore combiné, de produits azotés (ammoniaque, nitrates, nitrites), de substances organiques chlorées (AOX), de chlorures et de sodium issus de l'eau de Javel. Par ailleurs, la monochloramine est sujette à un phénomène de dégazage dans les tours aéroréfrigérantes, générant des pertes dans l'atmosphère. La concentration en chlore combiné résiduel à la sortie du condenseur est maintenue à environ 0,25 mg/L éq. $\mathrm{Cl}_{2}$.

Le traitement biocide par chloration massive à $\mathrm{pH}$ contrôlé est déclenché en cas de développement d'algues filamenteuses ou en secours du traitement à la monochloramine ou, en l'absence de traitement à la monochloramine, en cas de prolifération de micro-organismes pathogènes (cf. chapitre 7 sur les prélèvements d'eau et la source froide). Il fait appel à de l'eau de Javel injectée en une fois, à forte teneur, pour provoquer un choc chloré ; une concentration de $50 \mathrm{mg} / \mathrm{L}$ de chlore libre est généralement visée dans le circuit. Cette opération est pratiquée après avoir fermé les purges de l'aéroréfrigérant afin d'éviter le rejet massif de chlore libre ; une injection continue d'acide sulfurique pendant l'opération permet de maintenir le $\mathrm{pH}$ de l'eau du circuit entre 7,2 et 7,6 pour maîtriser l'entartrage (cf. figure 16). Pendant l'opération qui dure plusieurs heures, l'eau de Javel réagit avec la matière organique du circuit et se transforme en chlore combiné, en AOX (composés organohalogénés) et en THM (composés organohalogénés volatils dont principalement le chloroforme). Ces substances sont rejetées à l'ouverture de la purge dans l'ouvrage de rejet principal (cf. tableau III). La purge n'est ouverte que lorsque la concentration en chlore libre dans le bassin de l'aéroréfrigérant est inférieure à $0,1 \mathrm{mg} / \mathrm{L}$ éq. $\mathrm{Cl}_{2}$. Par ailleurs, l'eau de Javel est sujette à un phénomène de dégazage dans les tours aéroréfrigérantes, générant des pertes d'acide hypochloreux et de THM dans l'atmosphère.

Tableau II Le tableau suivant présente les limites journalières de rejets autorisés pour le traitement antitartre à Bugey (Arrêté du 6 août 2014 portant homologation de la décision $n^{\circ}$ 2014-DC-0443 de l'Autorité de sûreté nucléaire du 15 juillet 2014).

\begin{tabular}{|l|c|} 
& $\begin{array}{c}\text { FLUX JOURNALIER MAXIMAL } \\
\text { AJOUTÉ }\end{array}$ \\
\hline Sulfates $^{(1)}$ & $25000 \mathrm{~kg}$ \\
\hline $\begin{array}{l}\text { Antitartre } \\
\text { organiques }\end{array}$ & $\begin{array}{c}4500 \mathrm{~kg} \text { au maximum } 107 \text { jours par an } \\
3000 \mathrm{~kg} \text { le reste de l'année }\end{array}$ \\
\hline DCO & $\begin{array}{c}5800 \mathrm{~kg} \text { au maximum } 107 \text { jours par an } \\
3900 \mathrm{~kg} \text { le reste de l'année }\end{array}$ \\
\hline
\end{tabular}

(1) Les rejets de sulfates sont associés au traitement antitartre et au traitement biocide. 
Fig. 15 - La monochloramine utilisée pour le traitement biocide des circuits dits « fermés " de refroidissement avec des tours aéroréfrigérantes est produite in situ par mélange d'eau de Javel et d'ammoniaque. Elle est injectée dans le circuit à l'amont du condenseur.

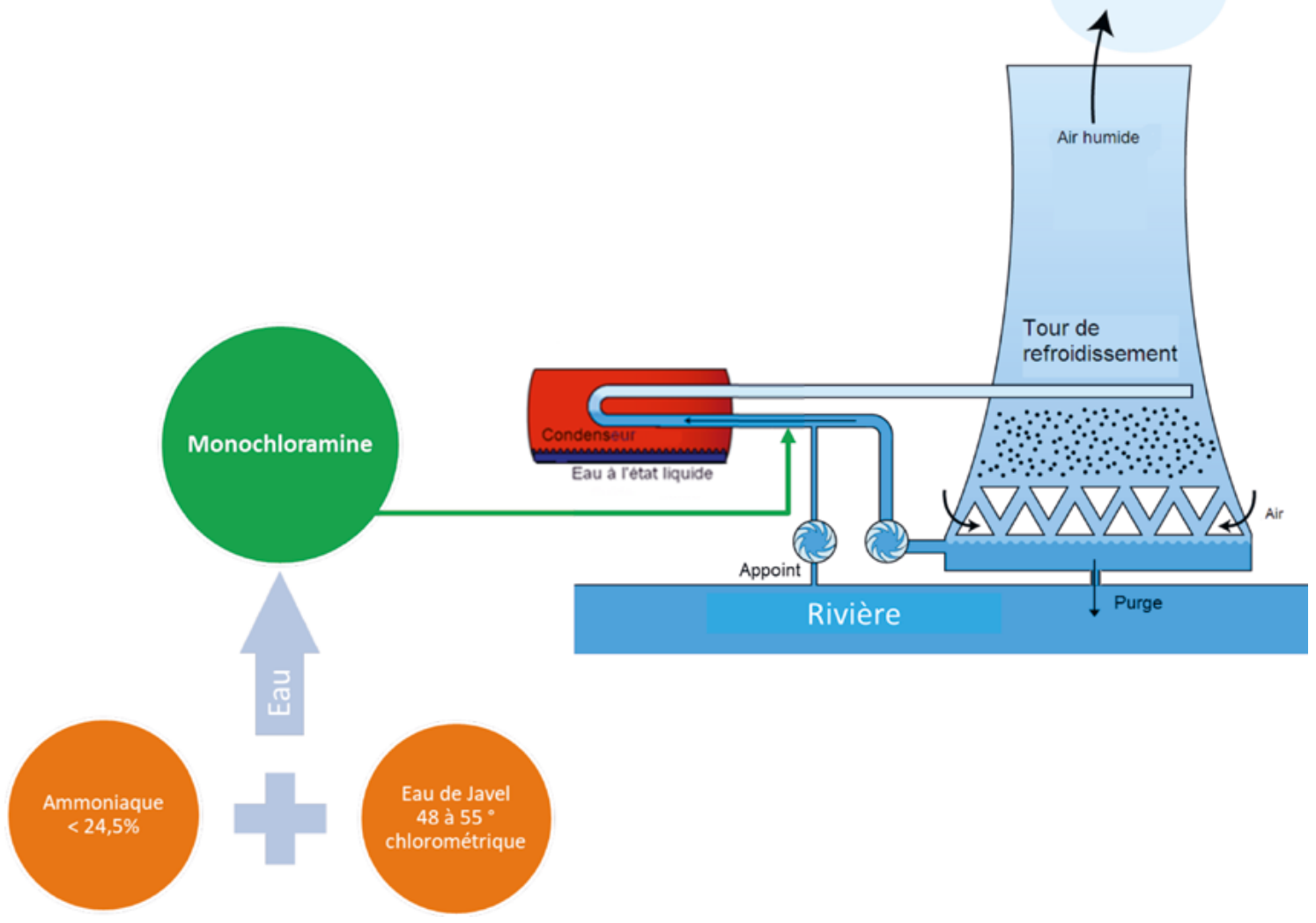

Tableau III Le tableau ci-dessous donne un exemple de limites journalières de rejet autorisées lors de la mise en œuvre d'un traitement biocide. Il s'agit d'un extrait de l'arrêté du 19 mars 2015 portant homologation de la décision ASN 2015-DC-0498 du 19 février 2015 concernant la centrale de St-Laurent sur la Loire (deux unités de $900 \mathrm{MWe}$ ).

\begin{tabular}{|l|c|c|}
\multicolumn{1}{|c|}{ SUBSTANCES } & $\begin{array}{c}\text { CHLORATION MASSIVE } \\
\text { À pH CONTRÓLÉ } \\
\text { FLUX 24 H AJOUTÉ }\end{array}$ & $\begin{array}{c}\text { TRAITEMENT À LA MONOCHLORAMINE } \\
\text { UNITÉS 1 ET 2 FLUX 24 H AJOUTÉ }\end{array}$ \\
\hline Ammonium & - & $70 \mathrm{~kg}$ \\
\hline Nitrates & - & $\begin{array}{c}1470 \mathrm{~kg} \\
1840 \mathrm{~kg} \text { en cas de traitement renforcé }\end{array}$ \\
\hline Nitrites & - & $\begin{array}{c}70 \mathrm{~kg} \\
(10 \% \text { des valeurs peuvent dépasser } 70 \mathrm{~kg} \\
\text { sans toutefois dépasser 230 } \mathrm{kg})\end{array}$ \\
\hline CRT & $100 \mathrm{~kg}$ & $\begin{array}{c}45 \mathrm{~kg} \\
65 \mathrm{~kg} \text { en cas de traitement renforcé }\end{array}$ \\
\hline AOX éq Cl 2 & $85 \mathrm{~kg}$ & $\begin{array}{c}15 \mathrm{~kg} \\
20 \mathrm{~kg} \text { en cas de traitement renforcé }\end{array}$ \\
\hline THM (chloroforme) & $9,5 \mathrm{~kg}$ & - \\
\hline
\end{tabular}

Ces traitements s'accompagnent de rejets de sulfates, de chlorures et de sodium réglementés avec les rejets provenant d'autres circuits (station de déminéralisation, réservoirs T, S et Ex)

Les purges des aéroréfrigérants principaux du CNPE de Civaux sont également équipées d'un traitement physique anti-amibien par rayonnements ultraviolets (UV) depuis 1999 pour la tranche 1 et depuis 2000 pour la tranche 2. Sur chaque purge, en période estivale, l'eau passe dans des canaux parallèles remplis de lampes monochromatiques montées en bancs, émettant des UV qui détruisent les micro-organismes. Le nettoyage de ces bancs est réalisé avec de l'acide phosphorique et génère des rejets de phosphates réglementés dans les décisions limites et modalités du site. 
Fig. 16 Le traitement biocide par chloration massive à pH contrôlé est utilisé en cas d'absence ou d'indisponibilité du traitement à la monochloramine. Il consiste en une injection ponctuelle d'eau de Javel associée à une injection continue d'acide sulfurique permettant de maîtriser l'entartrage du circuit pendant l'opération.

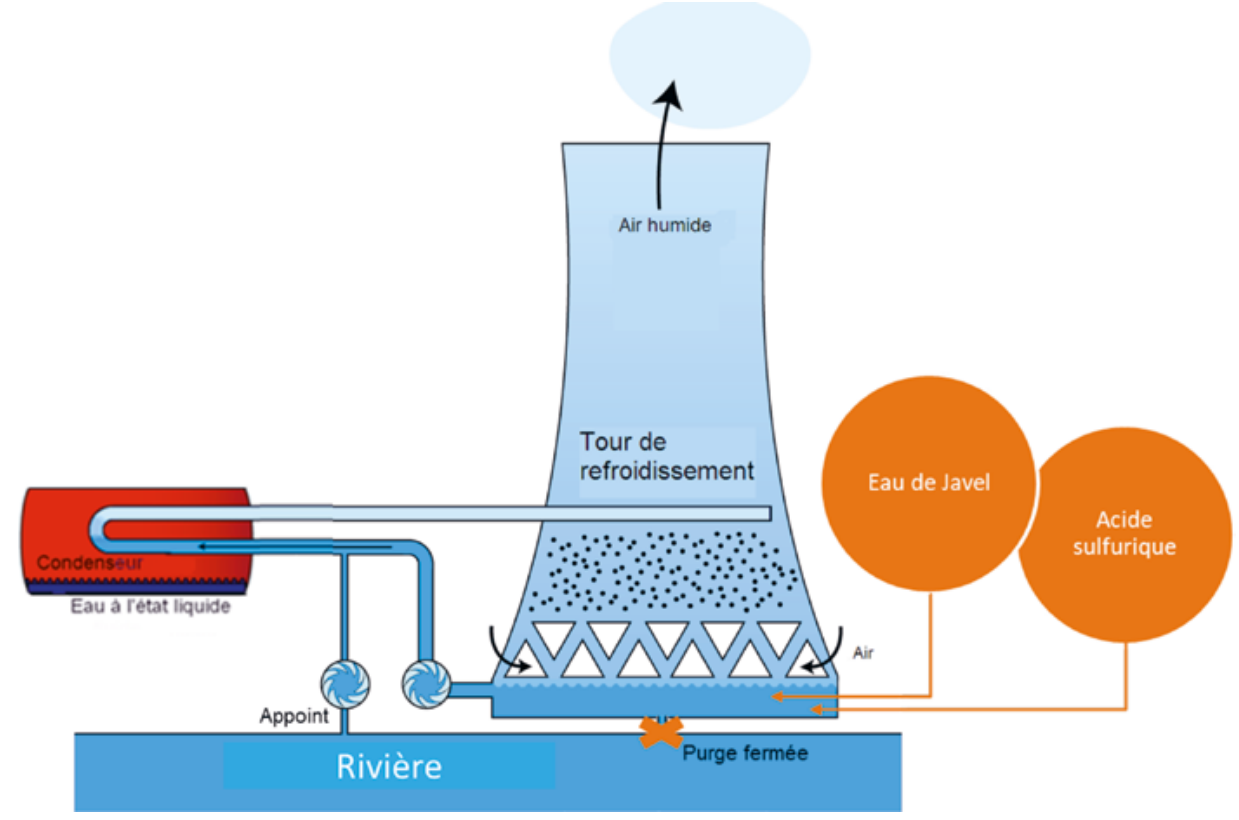

Traitement biocide des circuits ouverts

\section{de refroidissement des sites marins}

Pour lutter contre les salissures biologiques, l'eau de mer alimentant les circuits de refroidissement des sites marins est traitée, dès que la température de l'eau de mer est supérieure à $10^{\circ} \mathrm{C}$, à l'eau de Javel (hypochlorite de sodium) produite in situ par électrolyse de l'eau de mer. Ce traitement par électrochloration conduit à des rejets d'oxydants résiduels chlorés et bromés (généralement moins de 1000 kg par jour pour une unité de production) ainsi qu'à des rejets de composés organohalogénés dont le principal est le bromoforme (généralement moins de 100 kg par jour pour une unité de production).

\section{Production d'eau déminéralisée à partir d'eau douce brute}

Les stations de production d'eau déminéralisée se composent (cf. figure 17) :

- d'une unité de prétraitement qui peut être, selon les besoins, de différents types:

- décarbonatation et floculation,

- clarification et décantation ou flottation,

- filtration sur filtre à sable ;

- d'une unité de déminéralisation composée, selon les besoins:

- d'un déchloreur,

- de déminéraliseurs (résines échangeuses d'ions pour les cations et résines échangeuses d'ions pour les anions),

- dans certains cas, des déminéraliseurs à lits mélangés (résines anions/cations).

De plus, la mise en œuvre de membranes d'osmose inverse permet, par l'action mécanique d'une pression supérieure à la pression osmotique de l'effluent, de retenir la plus grande partie des sels dissous en solution. Dans le cadre de la production d'eau déminéralisée, l'étape d'osmose inverse ne se substitue pas à la déminéralisation par résines échangeuses d'ions, mais intervient comme une étape de déminéralisation supplémentaire, en tête de chaîne. Cette étape, mise en œuvre en amont des résines échangeuses d'ions, réduit la salinité de l'eau à déminéraliser, et permet par conséquent, d'augmenter la durée du cycle des résines. Il en résulte des volumes produits par cycle plus importants et une utilisation moyenne de régénérants moindre. Toutefois, les membranes d'osmose inverse sont sujettes à l'entartrage ainsi qu'au colmatage biologique et minéral pouvant requé-

Fig. 17 Les stations de production d'eau déminéralisée équipant les centrales françaises d'EDF sont équipées d'une unité de prétraitement et d'une unité de déminéralisation. Le schéma de principe ci-dessous en présente la configuration.

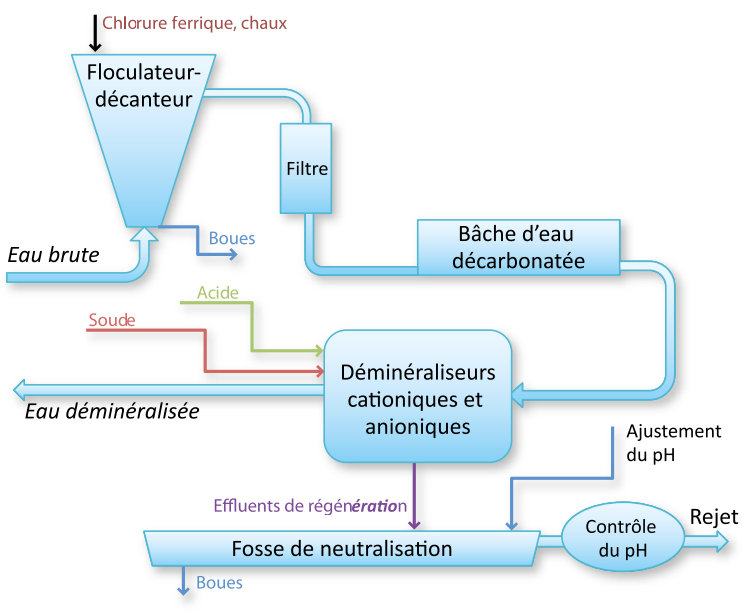


rir des nettoyages réguliers. Leur exploitation implique aussi nécessairement des pertes en eau sous forme de concentrats (de l'ordre de $25 \%$ du débit d'eau brute pompée). Cette adjonction peut être réalisée de manière fortuite lors d'une indisponibilité de production sur la station. Elle est mise en œuvre de manière pérenne à Cattenom, Tricastin et Flamanville.

Le fonctionnement d'une station de production d'eau déminéralisée produit :

- des boues provenant de l'unité de prétraitement;

- des effluents chimiques issus du lavage du déchloreur et des éventuelles membranes d'osmose inverse et de la régénération des résines échangeuses d'ions au moyen d'acide sulfurique ou chlorhydrique (résines cationiques) et de soude (résines anioniques).

Les effluents de régénération sont composés essentiellement de chlorures ou de sulfates, de sodium et de métaux ; ils sont orientés vers la fosse de neutralisation pour y être analysés et, le cas échéant, neutralisés, avant rejet (cf. tableau IV).

Tableau IV Le tableau ci-dessous donne un exemple de limites journalières de rejet autorisées associées à l'exploitation d'une station de production d'eau déminéralisée. Il s'agit d'un extrait de l'arrêté du 15 février 2008 portant homologation de la décision ASN 2008-DC-0090 du 10 janvier 2008 concernant à la centrale de Penly (deux unités de $1300 \mathrm{MWe})$.

\begin{tabular}{|l|c|}
\multicolumn{1}{c|}{ SUBSTANCES } & FLUX 24 H AJOUTÉ \\
\hline Sodium & $830 \mathrm{~kg}$ \\
\hline Chlorures & $1100 \mathrm{~kg}$ \\
\hline Fer & $56 \mathrm{~kg}$ \\
\hline Matières en suspension & $1800 \mathrm{~kg}$ \\
\hline
\end{tabular}

Les boues de décantation et de nettoyage des fosses de neutralisation sont selon leur composition, soit utilisées comme amendement agricole, soit traitées comme des déchets.

\section{Production d'eau déminéralisée par dessalement de l'eau de mer à Flamanville}

L'eau prélevée en mer subit:

- une pré-filtration afin d'éviter le colmatage des matériels situés en aval ;

- une filtration accompagnée d'une coagulation par injection de chlorure ferrique $\left(\mathrm{FeCl}_{3}\right)$ et éventuellement d'acide sulfurique pour ajustement du pH ;

- une ultrafiltration assurée par deux unités en parallèle dont le nettoyage est réalisé automatiquement au moyen d'eau de Javel ( $\mathrm{NaClO}$, neutralisée avec du bisulfite de sodium $\mathrm{NaHSO}_{3}$ avant évacuation vers la fosse de neutralisation) ou d'acide sulfurique $\left(\mathrm{H}_{2} \mathrm{SO}_{4}\right)$. Un nettoyage complémentaire est à réaliser environ 4 fois par an au moyen de soude et de bisulfite de sodium ou de détergents;

- un traitement par osmose inverse (avec injection de soude pour augmenter la rétention du bore par les membranes) permettant d'obtenir, d'une part, de l'eau douce et, d'autre part, un concentrat de sels (saumure) qui est rejeté ; ce traitement est accompagné :

- d'une injection de bisulfite de sodium $\left(\mathrm{NaHSO}_{3}\right)$ afin d'éliminer le chlore et l'oxygène présents dans l'eau de mer à traiter,

- d'un ajout de produits anti-incrustants pour empêcher la précipitation de composés tels que le carbonate de calcium et le sulfate de calcium.

Les membranes d'osmose se colmatent lentement sous l'effet des colloïdes et de la précipitation des sels. Pour restaurer la propreté des membranes, un nettoyage chimique au moyen d'une solution de soude et de bisulfite de sodium ou de détergents est nécessaire.

L'eau douce sortant des osmoseurs subit une déminéralisation finale (polishing) sur un lit mélangé de résines échangeuses d'ions. Les résines sont périodiquement régénérées par de la soude et de l'acide sulfurique et les effluents produits sont orientés vers la fosse de neutralisation pour contrôle avant rejet (cf. figure 18 et tableau $\mathrm{V}$ ).

Fig. 18 Le schéma de principe ci-dessous présente la configuration de la station de dessalement d'eau de mer à Flamanville. L'eau de mer prélevée subit un prétraitement,

une ultrafiltration et un traitement par osmose inverse permettant d'obtenir une eau douce présentant une qualité adaptée à son utilisation dans les différents circuits d'eau de refroidissement du réacteur.

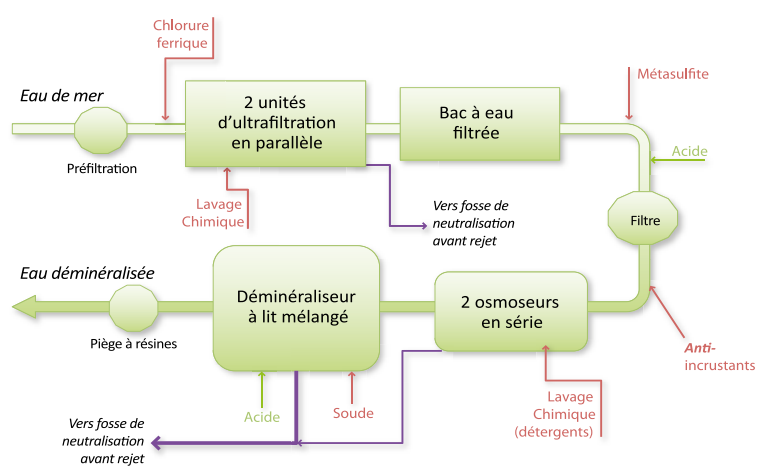

Tableau V Le tableau ci-dessous donne les ordres de grandeur des quantités de produits utilisés quotidiennement pour l'exploitation de l'unité de dessalement de l'eau de mer à Flamanville (environ $1500 \mathrm{~m}^{3} / \mathrm{j}$ ).

\begin{tabular}{|l|c|}
\multicolumn{1}{c|}{$\begin{array}{c}\text { SUBSTANCES REJETÉES } \\
\text { PAR L'UNITÉ }\end{array}$} & $\begin{array}{c}\text { QUANTITÉS } \\
\text { JOURNALIĖRES }\end{array}$ \\
\hline Chlorure ferrique & $80 \mathrm{~kg}$ \\
\hline Bisulfite de sodium & $90 \mathrm{~kg}$ \\
\hline Acide sulfurique & $720 \mathrm{~kg}$ \\
\hline Eau de Javel & $20 \mathrm{~L}$ \\
\hline Soude & $850 \mathrm{~kg}$ \\
\hline Anti-incrustant & $20 \mathrm{~kg}$ \\
\hline Détergents & $125 \mathrm{~kg}$ \\
\hline
\end{tabular}




\section{Substances utilisées pour les opérations de maintenance}

\section{Conditionnement chimique des générateurs de vapeur en phase d'arrêt de tranche}

Lorsque l'unité de production est mise à l'arrêt pour procéder au rechargement du combustible nucléaire, les générateurs de vapeur (côté secondaire) doivent être conditionnés pour assurer leur conservation et éviter leur endommagement pendant toute la durée de l'arrêt (plusieurs semaines). Cette conservation à l'arrêt peut être réalisée à sec ou en eau. En cas de conservation humide, l'eau est conditionnée au moyen d'un mélange d'hydrazine et d'ammoniaque ou d'hydrazine et d'éthanolamine ou de morpholine. En fin d'arrêt, deux cas de figure peuvent se présenter:

- soit les générateurs de vapeur sont exceptionnellement vidangés en l'état; l'eau de vidange contenant encore une forte teneur d'hydrazine (au moins $75 \mathrm{mg} / \mathrm{L}$ ) conduit à des rejets chimiques ;

- soit l'eau des générateurs de vapeur est conservée pour la phase de démarrage, ce qui évite les rejets.

Les autres parties du circuit secondaire (poste d'eau, condenseurs) sont vidangées en début d'arrêt et maintenues sous air avec une humidité relative inférieure à $40 \%$. Cette vidange de circuit en début d'arrêt de tranche produit environ $2000 \mathrm{~m}^{3}$ d'eau contenant de faibles concentrations d'hydrazine (50 à $100 \mu \mathrm{g} / \mathrm{L}$ ), d'ammoniaque (1 à $2 \mathrm{mg} / \mathrm{L}$ ) et d'éthanolamine (4 mg/L) ou de morpholine (6 mg/L) correspondant aux concentrations du circuit secondaire.

\section{Lessivage chimique des générateurs de vapeur à l'arrêt}

Le colmatage par des produits de corrosion issus du circuit secondaire a été constaté sur les générateurs de vapeur de certaines centrales. Ce phénomène pouvant affecter la tenue mécanique des tubes et impacter la sûreté, un lessivage chimique de ces matériels peut être nécessaire (cf. figure 19).

Cette opération de lessivage met en œuvre des substances chimiques différentes selon les procédés qualifiés par EDF (notamment éthylènediamine - EDA, acide éthylènediaminetétraacétique - EDTA, ammoniaque, hydrazine, acide oxalique, peroxyde d'hydrogène) qui vont dissoudre les oxydes métalliques (fer, cuivre, zinc) qui se sont déposés au fil du temps au niveau des plaques entretoises des générateurs de vapeur. Les effluents produits sont stockés dans des réservoirs prévus à cet effet et sont contrôlés. Selon leurs caractéristiques chimiques, les effluents liquides produits (plusieurs centaines de $\mathrm{m}^{3}$ ) sont ensuite transférés dans les réservoirs T pour y être contrôlés avant rejet (eaux de rinçage de l'opération), ou font l'objet d'un traitement spécifique permettant leur rejet ultérieur ou leur évacuation comme déchets. Les
Fig. 19 Un phénomène de colmatage est observé dans les générateurs de vapeur (GV) de certaines centrales. Ce phénomène correspond à l'obturation des trous qui permettent le passage de l'eau à travers les plaques entretoises de maintien des tubes des GV et est dû à la présence de dépôts dans ces passages d'eau. Des opérations de lessivage chimique peuvent être mises en œuvre pour remédier à ce phénomène. @EDF

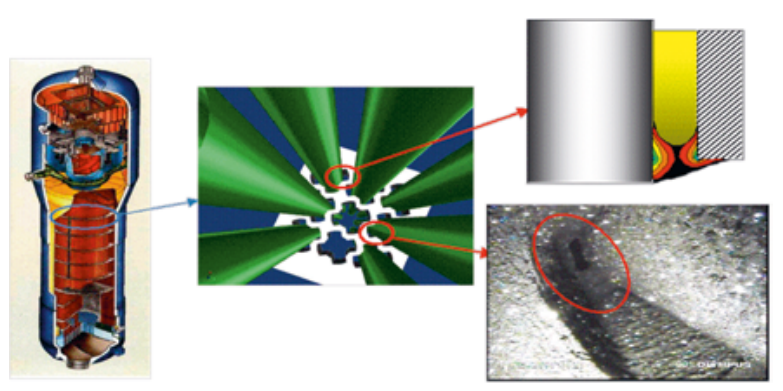

rejets de substances réglementées par les décisions limites et modalités du site se font dans le respect des autorisations réglementaires. Les substances non réglementées par les décisions limites et modalités du CNPE font l'objet d'une évaluation d'impact environnemental et de risques sanitaires transmise à l'ASN dans le dossier d'autorisation de l'opération, afin de démontrer l'absence d'impact environnemental et sanitaire de ces rejets.

\section{Nettoyage des échangeurs d'eau brute des circuits de réfrigération intermédiaires (circuits RRI, SRI, TRI)}

L'encrassement des échangeurs alimentés en eau brute (eau douce ou eau de mer) est inéluctable dans le temps et dégrade leurs performances thermiques, diminuant leur capacité à transférer l'énergie thermique. La nature des dépôts peut être d'origine organique (algues, boues) ou minérale (tartre, argile). Dans certains cas, ces dépôts peuvent contenir des traces d'hydrocarbures dues aux activités portuaires (cas de Gravelines). Le nettoyage peut être réalisé mécaniquement en démontant l'échangeur ou chimiquement. Les dépôts et les effluents produits sont, soit récupérés et traités comme déchets, soit rejetés après neutralisation dans les fosses de station de déminéralisation dans le respect des exigences réglementaires définies dans les décisions limites et modalités des sites concernés.

\section{Substances issues de l'usure des matériels ou d'impuretés des produits utilisés}

\section{Usures des tubes en laiton des condenseurs}

Les parois internes des tubes en laiton ${ }^{1}$ des condenseurs s'usent sous l'effet de l'érosion-corrosion provoquée par l'eau brute de refroidissement. La perte de matière se traduit par une diminution de l'épaisseur des tubes et conduit à des rejets de cuivre et de zinc. 
Seules quelques centrales refroidies en circuit dit « fermé » sont encore équipées de condenseurs dont les tubes sont totalement ou partiellement en laiton (cf. encart p. 159). Depuis 2011, plus aucune centrale refroidie en circuit ouvert ne possède de condenseurs en laiton.

Pour les centrales en circuit dit «fermé », les rejets journaliers par unité peuvent varier d'une dizaine à une centaine de $\mathrm{kg}$ pour le cuivre et de quelques kg à une cinquantaine de kg pour le zinc. L'usure dépend beaucoup de la qualité de l'eau brute qui peut être plus ou moins abrasive et corrosive selon les cours d'eau et selon les conditions météorologiques. Notons par ailleurs que la présence de cuivre soluble dans l'eau de refroidissement a un effet biocide vis-à-vis des amibes et évite leur développement dans les circuits (cf. chapitre 7 sur les prélèvements d'eau).

L'épaisseur des tubes de condenseurs est contrôlée périodiquement par des mesures par courants de Foucault. Ces contrôles montrent que les tubes en acier inoxydable et en titane ne présentent pas d'usure significative. Ces condenseurs ne donnent donc pas lieu à des rejets de métaux.

\section{Impuretés des produits commerciaux utilisés pour le conditionnement chimique et le traitement}

Certains produits chimiques commerciaux utilisés pour le traitement des circuits (acide sulfurique, soude caustique, acide chlorhydrique) renferment des impuretés telles que le plomb et le mercure. Pour réduire au maximum le rejet de ces substances très toxiques, l'exploitant impose dans le cahier des charges transmis aux fournisseurs, des spécifications adaptées.

\section{Substances chimiques exogènes}

Les substances chimiques présentes dans l'eau prélevée en amont de la centrale et qui ne font que transiter par les circuits de refroidissement (sels dissous, métaux lourds...) se retrouvent aussi dans l'ouvrage de rejet principal. Les circuits dits «fermés » refroidis par aéroréfrigérants tendent à concentrer ces substances au rejet du fait de l'évaporation de l'eau (facteur de concentration de 1,5 environ). Ce phénomène de surconcentration est pris en compte dans les études d'impact (cf. chapitre 9 sur la maîtrise des impacts).

\subsection{COLLECTE ET TRAITEMENT DES EFFLUENTS CHIMIQUES LIQUIDES}

\section{Collecte vers I'ouvrage de rejet principal}

À l'exception des eaux de pluie collectées par certains émissaires, tous les effluents, qu'ils soient radioactifs ou non, sont orientés vers un ouvrage de rejet unique qui constitue le rejet principal du site. Cet ouvrage collecte les retours d'eau brute (eau douce ou eau de mer) des circuits de refroidissement (condenseurs des turbo-alternateurs, purges des tours aéroréfrigérantes, échangeurs) dans lesquels les effluents sont prédilués. À cette fin, un débit minimal d'eau brute est assuré en permanence dans cet ouvrage de rejet (cf. figure 20).

Par l'ouvrage de rejet principal, sont rejetées les substances chimiques en provenance:

- des vidanges des réservoirs T (capacité unitaire 300 à $750 \mathrm{~m}^{3}$ ) collectant les effluents radioactifs (10 à 20 vidanges par an et par réacteur). Ces réservoirs contiennent, en plus des substances radioactives, des substances chimiques telles que:

- acide borique, lithine, hydrazine, phosphates, détergents provenant du lavage du linge contaminé ou des douches,

- les produits de conditionnement chimique du circuit secondaire (morpholine ou éthanolamine, hydrazine, ammoniaque, et leurs dérivés azotés tels que nitrates et nitrites) si les réservoirs ont collecté les purges des générateurs de vapeur,

- les matières en suspension (boues) voire quelques métaux à l'état de trace dus à l'usure des circuits ;

- des vidanges des réservoirs Ex, (capacité unitaire 500 à $1000 \mathrm{~m}^{3}$ ) collectant les eaux d'exhaure des salles des machines (40 à 150 vidanges par an et par réacteur). Ces réservoirs renferment essentiellement: - les produits de conditionnement chimique du circuit secondaire et du circuit de production de vapeur auxiliaire (morpholine ou éthanolamine, hydrazine, ammoniaque, et leurs dérivés azotés tels que nitrates et nitrites),

- les matières en suspension (issues de la récupération des eaux brutes de désurchauffe des fonds «basse-pression » des turbines) voire quelques métaux à l'état de trace dus à l'usure des circuits,

- les phosphates issus des vidanges des circuits de refroidissement intermédiaire ;

- des purges continues des aéroréfrigérants, qui contiennent les substances chimiques liées aux traitements antitartre et biocide lorsqu'ils sont pratiqués, et à l'usure des tubes de condenseurs si ces derniers sont en laiton;

- des retours d'eau brute des condenseurs refroidis en circuit ouvert qui peuvent être chargés de substances liées au traitement antisalissure sur les sites marins ;

- des vidanges des fosses de neutralisation (170 à $700 \mathrm{~m}^{3}$ ) de la station de déminéralisation comportant notamment les sels issus de la régénération des résines échangeuses d'ions (sulfates ou chlorures);

- des sorties des stations d'épuration des eaux usées STEP (eaux vannes);

- des sorties des déshuileurs installés pour la collecte des eaux susceptibles d'être polluées par de l'huile ou des hydrocarbures ;

- des eaux de pluie collectées par les toitures des bâtiments et les voiries. 
Fig. 20 Le schéma simplifié ci-dessous présente le principe de collecte des effluents liquides. Tous les effluents, radioactifs ou non, sont orientés vers un ouvrage de rejet unique qui constitue le rejet principal du site. Dans cet ouvrage, les effluents sont pré-dilués et contrôlés avant d'être rejetés.

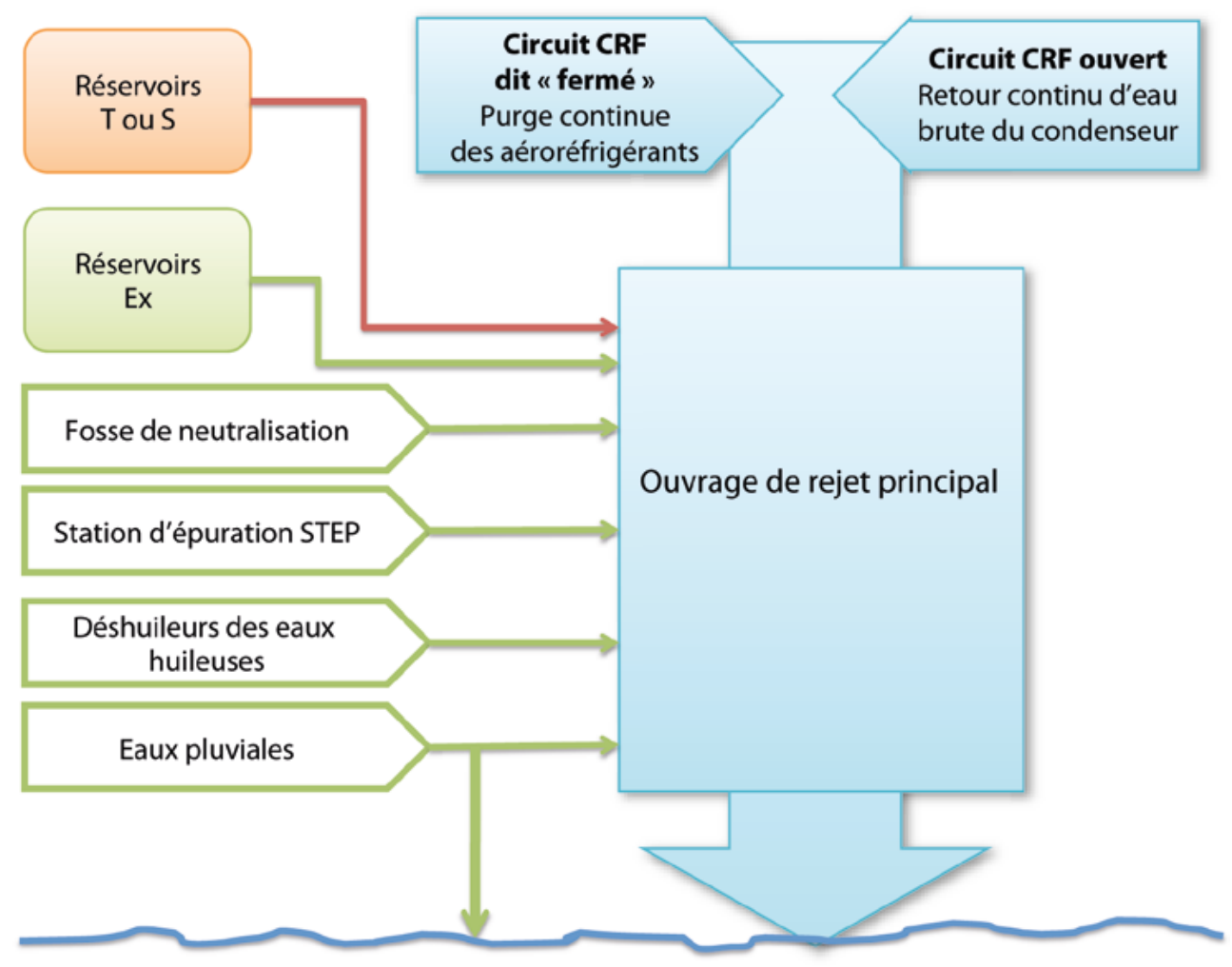

Rivière ou mer

\section{Traitement des effluents chimiques}

Des actions sont mises en œuvre au quotidien par l'exploitant pour réduire autant que possible les rejets de substances chimiques. Celles-ci visent en premier lieu à réduire la production d'effluents à la source en limitant notamment les fuites d'eau sur les circuits puis en agissant sur le traitement des effluents avant rejet (cf. chapitre 9 sur la maîtrise des impacts).

\section{Réduction des rejets d'acide borique}

Les effluents radioactifs borés issus du circuit primaire peuvent être, après traitement, recyclés, c'est-à-dire réutilisés comme eau d'appoint au circuit primaire. Mais le recyclage se heurte à des difficultés en raison de la teneur en silice présente à l'état de trace dans l'effluent à recycler. Comme celui-ci subit un traitement sur évaporateur qui concentre l'acide borique mais aussi la silice, la teneur en silice dans l'eau à recycler peut atteindre la limite fixée par les spécifications chimiques définies pour l'eau d'appoint au réacteur. Dans ce cas, le recyclage de l'eau borée n'est pas possible et elle doit être rejetée (cf. figure 21). C'est ce qui explique en partie les variations importantes des rejets d'acide borique entre sites. L'acide borique non recyclé est enfûté en coque béton ou incinéré à l'usine Centraco de Marcoule comme un déchet radioactif.
Fig. 21 Lorsqu'ils ne peuvent pas être recyclés, les effluents contenant de l'acide borique, issus du circuit primaire, doivent être rejetés. Les rejets annuels moyens d'acide borique ramenés à une unité de production sont inférieurs à 5 tonnes depuis une dizaine d'années.

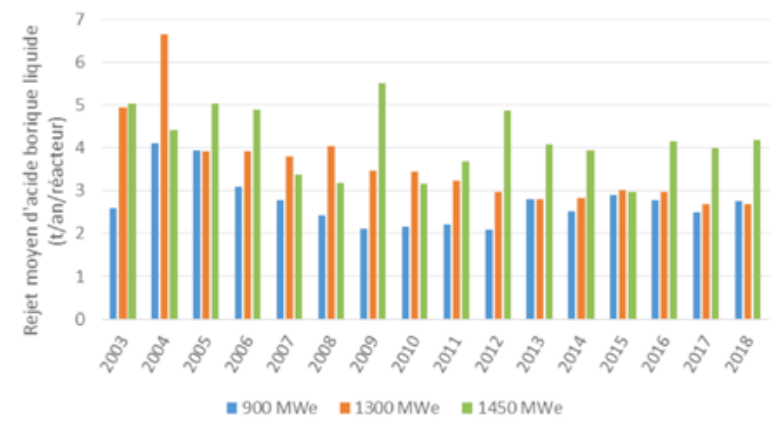

\section{Élimination de l'hydrazine}

Lorsque la centrale est en fonctionnement, l'hydrazine du circuit secondaire (eau-vapeur) chemine par les purges des circuits jusqu'aux réservoirs d'entreposage T ou Ex d'entreposage avant rejet. Dans ces réservoirs, l'hydrazine se décompose au contact de l'oxygène de l'air ambiant. De ce fait, les rejets d'hydrazine sont faibles (quelques centaines de grammes par réacteur et par an).

En arrêt de tranche, le conditionnement chimique à l'hydrazine des générateurs de vapeur (côté secondaire) 
et du poste d'eau pouvait être à l'origine de rejets plus importants (jusqu'à quelques dizaines de kilos d'hydrazine par jour) lors de la vidange des circuits. Pour réduire ces rejets, les actions suivantes ont été prises :

- le poste d'eau et les générateurs de vapeur sont dorénavant conservés de manière privilégiée à sec sous air avec une humidité relative inférieure à $40 \%$, après avoir été vidangés en début d'arrêt de tranche alors que la teneur en hydrazine dans le circuit secondaire est faible (50 à $100 \mu g / L$ ) ;

- si la conservation à sec des générateurs de vapeur n'est pas possible, la mise en œuvre préférentielle du cracking thermique de l'hydrazine résiduelle issue du conditionnement humide permet, lors de la montée en température au redémarrage du réacteur, la décomposition thermique de l'hydrazine dans les GV et évite les rejets liquides ; l'azote issu de cette opération est alors rejeté à l'atmosphère ;

- en dernier recours, l'hydrazine des effluents produits par la vidange des générateurs de vapeur en fin d'arrêt (300 et $400 \mathrm{~m}^{3}$ à la concentration d'au moins $75 \mathrm{mg} / \mathrm{L}$ ) est éliminée en quasi-totalité dans les réservoirs d'entreposage par un bullage à l'air comprimé ou par la mise en brassage prolongé des réservoirs, avec l'ajout possible de quelques centaines de grammes de sulfate de cuivre jouant le rôle de catalyseur de la réaction.

Par ailleurs, les matériels (pompes d'injection...) véhiculant de l'hydrazine concentrée font l'objet d'une attention particulière afin de déceler toute fuite de produit et d'y remédier.

En définitive, les rejets d'hydrazine ne représentent plus au total que quelques centaines de grammes par réacteur et par an. Ainsi, les rejets liquides d'hydrazine ont été divisés par un facteur 30 en 12 ans (cf. figure 22).

\section{Réduction des rejets d'azote total (hors hydrazine, mor-} pholine, éthanolamine)

Le conditionnement chimique du circuit secondaire et le traitement biocide des circuits dits «fermés » de refroidissement engendrent des rejets de substances azotées telles que l'ammoniaque, les nitrates et les nitrites. L'azote de l'hydrazine $\left(\mathrm{N}_{2} \mathrm{H}_{4}\right)$, de la morpholine $\left(\mathrm{C}_{4} \mathrm{H}_{9} \mathrm{NO}\right)$ et de l'éthanolamine $\left(\mathrm{C}_{2} \mathrm{H}_{7} \mathrm{NO}\right)$ n'est pas pris en compte dans le vocable « azote » ou « azote total »; celui-ci étant considéré au travers des teneurs en hydrazine, en morpholine et en éthanolamine des effluents (cf. chapitre 11 §4).

L'ammoniaque $\left(\mathrm{NH}_{4} \mathrm{OH}\right)$ dans les effluents provient principalement :

- de la décomposition thermique de l'hydrazine ;

- de l'ammoniaque utilisée pour le conditionnement chimique du circuit secondaire sur les unités sans alliages cuivreux ;

- du traitement biocide à la monochloramine des circuits de refroidissement dits «fermés ».

L'ammoniaque se concentre fortement dans les purges des pompes à vide extrayant les gaz incon-
Fig. 22 Les rejets annuels moyens d'hydrazine ont diminué d'un facteur 30 en 12 ans, grâce à la réduction à la source des effluents, à la mise en œuvre préférentielle d'une conservation sèche des matériels à l'arrêt ou au cracking thermique de l'hydrazine résiduelle issue du conditionnement humide des générateurs de vapeur à l'arrêt, ainsi qu'à la destruction de l'hydrazine contenue dans les réservoirs d'entreposage des effluents par bullage à l'air. Aujourd'hui, les rejets d'hydrazine sont faibles, de l'ordre de quelques centaines de grammes par an et par réacteur en moyenne.

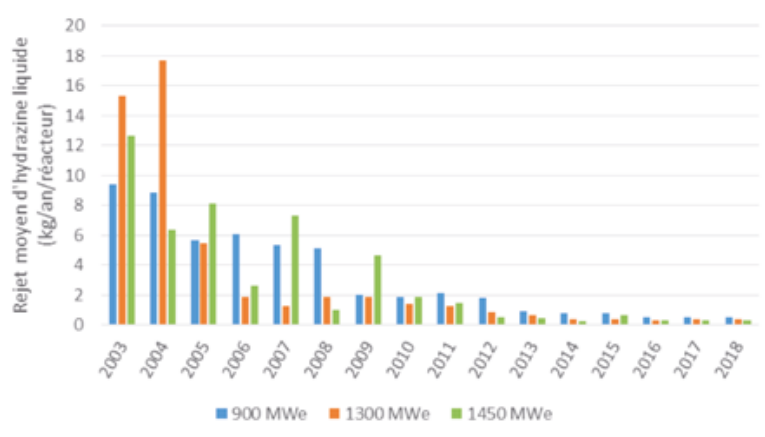

densables du circuit secondaire " eau-vapeur». Ces purges contribuent pour une grande part au rejet d'ammoniaque d'une centrale nucléaire. Ces rejets peuvent être réduits en pratiquant le recyclage des purges des pompes à vide au condenseur. Mais ce recyclage pouvant entraîner une " pollution chimique » du circuit secondaire préjudiciable à la bonne tenue des matériels, des modifications sont réalisées pour sécuriser sa mise en œuvre sur le palier 1450 MWe.

Les nitrates $\left(\mathrm{NO}_{3}^{-}\right)$et les nitrites $\left(\mathrm{NO}_{2}^{-}\right)$sont principalement produits par le traitement biocide à la monochloramine des circuits de refroidissement dits «fermés ». Les rejets de nitrates peuvent être réduits en optimisant les paramètres de traitement (injection au plus juste des quantités de réactifs en fonction du risque de colonisation des micro-organismes pathogènes, traitement séquentiel). L'expérience montre que les pics de rejet de nitrites surviennent lorsque le traitement est lancé pendant la phase de redémarrage de l'unité. Afin d'éviter ces pics de rejet, il est préconisé d'attendre que l'unité ait atteint la pleine puissance avant de démarrer le traitement biocide. Les nitrates et les nitrites sont aussi présents dans les eaux d'exhaure des salles des machines où ils se forment par oxygénation des produits azotés contenus dans ces eaux, notamment de l'ammoniaque.

\section{Réduction des rejets de détergents}

Les détergents proviennent du circuit de laverie du linge contaminé. Ces effluents ne font généralement l'objet que d'une filtration qui n'a pas d'efficacité vis-à-vis des détergents. Par ailleurs, la composition des détergents n'est pas précisément fournie par le fabricant, mais on sait qu'ils sont 
Tableau VI La rénovation des condenseurs, engagée par EDF depuis plusieurs années, a permis de réduire significativement les rejets de cuivre et de zinc sur tous les bassins hydrographiques.

\begin{tabular}{|c|c|c|c|c|}
\hline BASSIN HYDROGRAPHIQUE & SEINE & LOIRE & MOSELLE & RHÔNE \\
\hline $\begin{array}{l}\text { Réduction des rejets de cuivre et zinc par les condenseurs } \\
\text { en laiton }{ }^{1} \text { en } \% \text {, de } 1990 \text { à } 2018\end{array}$ & $\cong 100 \%$ & $\cong 77 \%$ & $\cong 87 \%$ & $\cong 100 \%$ \\
\hline
\end{tabular}

Nota: II nexiste pas de techniques industriellement et économiquement utilisables pour retenir ou piéger ces métaux dans les eaux de refroidissement en raison de leur très faible concentration dans les rejets.

Fig. 23 Le schéma ci-dessous présente le principe de la STEP Sud de Flamanville (800 éq-hab) dans laquelle les eaux usées sont traitées par un procédé biologique basé sur des cultures bactériennes fixées sur des membranes filtrantes et activé par oxygénation.

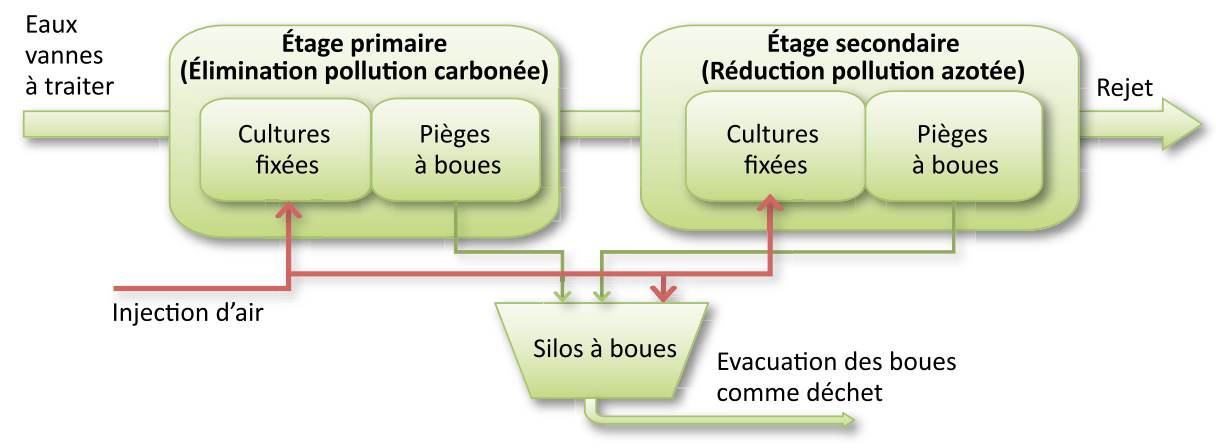

biodégradables, sans EDTA, ni NTA, ni phosphates. Des efforts sont réalisés afin de limiter autant que possible l'utilisation des détergents. Les mesures de propreté radiologique mises en œuvre dans les centrales depuis le début des années 2000 et une gestion efficace des équipements ont permis de réduire les quantités de linge à laver ainsi que la fréquence de lavage des sols.

\section{Réduction des rejets de métaux liés à l'usure des tubes de condenseurs en laiton}

À l'origine, le parc nucléaire en exploitation (19 sites) comportait des installations avec des condenseurs dont les tubes pouvaient être soit en titane (bord de mer), soit en inox (sites fluviaux récents, Golfech, Chooz, Civaux), soit en laiton pour les autres sites.

Depuis les années 1990, la plupart des condenseurs en laiton ont été rénovés soit en totalité par des tubes en acier inoxydable, soit partiellement par des tubes en titane ou en acier inoxydable (cf. encart p. 159). Cette rénovation engagée a permis de réduire significativement les rejets de cuivre et de zinc sur tous les bassins hydrographiques (cf. tableau VI).

\section{Traitement des eaux usées ou eaux vannes (STEP)}

Les eaux usées d'origine domestique (sanitaires, eaux vannes) sont collectées par un réseau particulier puis dirigées vers une station d'épuration (STEP) avant rejet. Le traitement est assuré biologiquement par culture bactérienne et oxygénation. Les eaux épurées sont rejetées tandis que les boues récupérées dans les bassins de décantation sont éliminées comme déchets ou consommées in situ par des lits de roseaux. Sur certains sites nucléaires (Nogent), les eaux usées sont orientées vers le réseau urbain de collecte pour y être traitées.

À la centrale de Flamanville, les eaux usées sont d'abord filtrées sur un tamis rotatif de maille $0,75 \mathrm{~mm}$ afin d'éliminer les sables et éléments grossiers; elles sont ensuite dirigées vers deux filières de traitement fonctionnant en parallèle. Une première filière assure une épuration biologique ; elle dispose d'une capacité de traitement de 800 équivalents-habitants et permet de traiter un volume journalier d'effluent jusqu'à $120 \mathrm{~m}^{3}$ (cf. figure 23). Une seconde filière, d'une capacité de 1000 équivalents-habitants, épure le volume restant dans un réacteur biologique à membranes (microfiltration 0,4 $\mu \mathrm{m}$ ). Les rejets de cette station d'épuration sont donnés au tableau VII.

Tableau VII Le tableau ci-dessous présente les limites de rejets autorisées pour la station d'épuration des eaux usées de Flamanville (station sud). Sa capacité de traitement est de 1800 équivalents-habitants $(\mathrm{EQH})$ répartis sur deux filières $(1000 \mathrm{EQH}$ en traitement membranaire et $800 \mathrm{EQH}$ en traitement cultures fixées) (décision ASN n²018-DC-0640 du 19 juillet 2018).

\begin{tabular}{|l|c|}
\multicolumn{1}{c|}{ PARAMÈTRES } & $\begin{array}{c}\text { CONCENTRATIONS } \\
\text { INSTANTANÉES } \\
\text { À RESPECTER SUR } \\
\text { DES MESURES RÉALISÉES } \\
\text { MENSUELLEMENT }\end{array}$ \\
\hline Phosphore total & - \\
\hline Azote global & - \\
\hline DBO5 & $30 \mathrm{mg} / \mathrm{L}$ \\
\hline DCO & $120 \mathrm{mg} / \mathrm{L}$ \\
\hline MES & $30 \mathrm{mg} / \mathrm{L}$ \\
\hline
\end{tabular}




\begin{tabular}{|c|c|c|c|}
\hline \multicolumn{4}{|c|}{ Rénovation des condenseurs en laiton } \\
\hline \multicolumn{4}{|c|}{$\begin{array}{l}\text { Depuis les années 1990, les condenseurs ont été rénovés; les tubes en laiton ont été remplacés, en partie ou en totalité, } \\
\text { par des tubes en acier inoxydable ou en titane (situation mi-2019), permettant de réduire significativement les rejets } \\
\text { de cuivre et de zinc sur tous les bassins hydrographiques. }\end{array}$} \\
\hline $\begin{array}{l}\text { BASSIN } \\
\text { DE LA LOIRE }\end{array}$ & $\begin{array}{l}\text { TAUX DE TUBES } \\
\text { DE CONDENSEURS } \\
\text { EN LAITON }\end{array}$ & $\begin{array}{l}\text { TAUX DE TUBES } \\
\text { DE CONDENSEURS EN ACIER } \\
\text { INOXYDABLE }\end{array}$ & $\begin{array}{l}\text { TAUX DE TUBES } \\
\text { DE CONDENSEURS } \\
\text { EN TITANE }\end{array}$ \\
\hline \multicolumn{4}{|l|}{ Belleville } \\
\hline Unité 1 & $55 \%$ & $45 \%$ depuis 2014 & \\
\hline Unité 2 & $65 \%$ & $35 \%$ & \\
\hline \multicolumn{4}{|l|}{ Dampierre } \\
\hline Unité 1 & & 100 \% depuis 1990 & \\
\hline Unité 2 & $80 \%$ & & $20 \%$ \\
\hline Unité 3 & & 100 \% depuis 1995 & \\
\hline Unité 4 & $80 \%$ & $20 \%$ & \\
\hline \multicolumn{4}{|l|}{ Saint-Laurent } \\
\hline Unité 1 & & $50 \%$ depuis 2009 & $50 \%$ depuis 2005 \\
\hline Unité 2 & & 100 \% depuis 2011 & \\
\hline \multicolumn{4}{|l|}{ Chinon } \\
\hline Unité 1 & & $100 \%$ depuis 2009 & \\
\hline Unité 2 & & $100 \%$ depuis 2010 & \\
\hline Unité 3 & & $100 \%$ depuis 2009 & \\
\hline Unité 4 & & $100 \%$ depuis 2010 & \\
\hline $\begin{array}{c}\text { BASSIN } \\
\text { DU RHÔNE }\end{array}$ & $\begin{array}{l}\text { TAUX DE TUBES } \\
\text { DE CONDENSEURS } \\
\text { EN LAITON }\end{array}$ & $\begin{array}{l}\text { TAUX DE TUBES } \\
\text { DE CONDENSEURS EN ACIER } \\
\text { INOXYDABLE }\end{array}$ & $\begin{array}{l}\text { TAUX DE TUBES } \\
\text { DE CONDENSEURS } \\
\text { EN TITANE }\end{array}$ \\
\hline \multicolumn{4}{|l|}{ Bugey } \\
\hline Unité 2 & & 100 \% depuis 1991 & \\
\hline Unité 3 & & $100 \%$ depuis 1990 & \\
\hline Unité 4 & & 100 \% depuis 1999 & \\
\hline Unité 5 & & 100 \% depuis 1993 & \\
\hline \multicolumn{4}{|l|}{ Saint-Alban } \\
\hline Unité 1 & & $100 \%$ depuis 2007 & \\
\hline Unité 2 & & $100 \%$ depuis 2008 & \\
\hline \multicolumn{4}{|l|}{ Cruas } \\
\hline Unité 1 & & $40 \%$ depuis 2017 & $60 \%$ depuis 2004 \\
\hline Unité 2 & & $40 \%$ depuis 2017 & $60 \%$ depuis 2004 \\
\hline Unité 3 & & $40 \%$ depuis 2016 & $60 \%$ depuis 2004 \\
\hline Unité 4 & & $40 \%$ depuis 2018 & $60 \%$ depuis 2003 \\
\hline \multicolumn{4}{|l|}{ Tricastin } \\
\hline Unité 1 & & $100 \%$ depuis 1994 & \\
\hline Unité 2 & & 100 \% depuis 1994 & \\
\hline Unité 3 & & 100 \% depuis 1992 & \\
\hline Unité 4 & & 100 \% depuis 1993 & \\
\hline $\begin{array}{l}\text { BASSIN DE LA } \\
\text { MOSELLE }\end{array}$ & $\begin{array}{l}\text { TAUX DE TUBES } \\
\text { DE CONDENSEURS EN } \\
\text { LAITON }\end{array}$ & $\begin{array}{c}\text { TAUX DE TUBES } \\
\text { DE CONDENSEURS } \\
\text { EN ACIER INOXYDABLE }\end{array}$ & $\begin{array}{c}\text { TAUX DE TUBES DE } \\
\text { CONDENSEURS EN TITANE }\end{array}$ \\
\hline \multicolumn{4}{|l|}{ Cattenom } \\
\hline Unité 1 & & & $100 \%$ depuis 2016 \\
\hline Unité 2 & & & 100 \% depuis 2017 \\
\hline Unité 3 & & & $100 \%$ depuis 2019 \\
\hline Unité 4 & & & $100 \%$ depuis 2018 \\
\hline $\begin{array}{c}\text { BASSIN } \\
\text { DE LA SEINE }\end{array}$ & $\begin{array}{l}\text { TAUX DE TUBES } \\
\text { DE CONDENSEURS } \\
\text { EN LAITON }\end{array}$ & $\begin{array}{l}\text { TAUX DE TUBES } \\
\text { DE CONDENSEURS EN ACIER } \\
\text { INOXYDABLE }\end{array}$ & $\begin{array}{l}\text { TAUX DE TUBES } \\
\text { DE CONDENSEURS } \\
\text { EN TITANE }\end{array}$ \\
\hline \multicolumn{4}{|l|}{ Nogent-sur-Seine } \\
\hline Unité 1 & & 100 \% depuis 1998 & \\
\hline Unité 2 & & 100 \% depuis 1999 & \\
\hline
\end{tabular}




\section{Traitement des eaux huileuses}

Même rejetés en faible quantité, les hydrocarbures ont un impact visuel très marqué dans l'environnement (irisations à la surface de l'eau). Pour éviter les rejets huileux, les circuits présentant un risque de fuite d'hydrocarbures (circuit d'huile du groupe turbo-alternateur, réservoirs de gasoil des groupes électrogènes de secours...) sont équipés de rétentions étanches et les réseaux d'égouts situés à proximité de ces matériels sont orientés vers un dispositif de traitement approprié tel que des déshuileurs (cf. figure 24).

Les réseaux de collecte des eaux pluviales des parkings extérieurs au site sont également équipés de rétentions et de systèmes de traitement (déshuileurs, débourbeurs) pouvant faire face à l'afflux d'eau dû à un gros orage. Ceci permet d'éviter le rejet de matières en suspension et d'hydrocarbures qui imprègnent les parkings de voitures.

Des mesures sont réalisées périodiquement à la sortie des systèmes de traitement afin de s'assurer qu'il n'y a pas de traces d'hydrocarbures supérieures à la limite réglementaire (5 ou $10 \mathrm{mg} / \mathrm{L}$ dans les effluents rejetés, selon le type d'effluents et les décisions limites et modalités des CNPE).

Fig. 24 Les eaux susceptibles de contenir des hydrocarbures sont, avant leur rejet, traitées par des dispositifs adaptés,

les déshuileurs, permettant de piéger les hydrocarbures. Ces dispositifs séparent les hydrocarbures et l'eau selon le schéma de principe présenté ci-dessous, en utilisant la densité des hydrocarbures inférieure à celle de l'eau (source : Eau du Grand Reims). Source : Techneau.

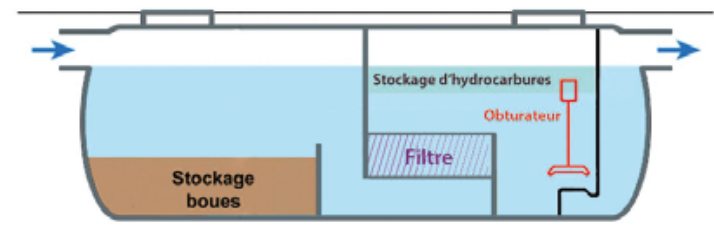

\subsection{REJETS CHIMIQUES LIQUIDES}

\section{Contrôle des rejets}

Les contrôles effectués sur les effluents chimiques par l'exploitant découlent directement des impositions réglementaires qui fixent notamment:

- la localisation, la nature, la technique de mesure, la périodicité ;

- les règles de comptabilisation des rejets ;

- les limites à ne pas dépasser ;

- les modalités de transmission des résultats de mesure à l'administration et au public.

Ces contrôles portent tant sur les concentrations des substances dans l'émissaire de rejet que sur les débits de rejet (contrôle des flux de rejet). La nature et la fréquence des contrôles tiennent compte des enjeux environnementaux en termes de quantité rejetée et de toxicité de la substance (cf. figure 25).

Les contrôles sont définis en distinguant:

- les réservoirs ou capacités de collecte (réservoirs $T, S$ et Ex, fosse de neutralisation). Pour certaines substances, les résultats d'analyse doivent être connus avant chaque rejet car ils déterminent les conditions de rejet (contrôle a priori) ; pour d'autres, les résultats n'interviennent qu'une fois le rejet effectué, au titre de la comptabilisation des flux de rejet journalier ou annuel (contrôle a posteriori). Les analyses sur les effluents sont réalisées après avoir mis en brassage le réservoir afin d'obtenir un échantillon représentatif sur lequel seront effectuées les analyses requises. Les quantités de substances rejetées (flux) sont déterminées d'après les analyses de concentration, les volumes des réservoirs ou les débits de rejet associé au temps de rejet. Le réservoir ne peut être rejeté que si les conditions réglementaires de rejet sont respectées (cf. § 4.5);

- les émissaires des stations d'épuration des eaux usées (STEP) et de déminéralisation, des purges des aéroréfrigérants ainsi que du traitement des eaux huileuses et de la collecte des eaux pluviales;

- l'ouvrage de rejet principal où le contrôle est réalisé :

- par des mesures en continu de paramètres physico-chimiques. L'ouvrage de rejet principal des centrales implantées en bord de rivière est équipé d'une station multiparamètres permettant de relever en continu, le $\mathrm{pH}$, la température, l'oxygène dissous et la conductivité,

- par des prélèvements périodiques sur lesquels des analyses de laboratoire sont opérées,

- en déterminant le débit de rejet par mesure ou par calcul d'après les paramètres de fonctionnement des installations (cf. chapitre 7 sur les prélèvements d'eau).

Les analyses chimiques sont effectuées par le laboratoire "Effluents» de la centrale en référence à des normes (cf. chapitre 11 sur la métrologie environnementale). Certaines analyses peuvent être sous-traitées à des laboratoires extérieurs après information préalable de l'ASN. Les résultats des mesures sont consignés dans les synthèses mensuelles et annuellement dans le registre national des émissions ainsi que dans le rapport environnemental du site, communiqués à l'administration et au public (cf. chapitre 4 sur l'information du public et chapitre 6 sur le rôle de l'administration).

\section{Contrôle des rejets de cuivre et de zinc des condenseurs en laiton}

Les rejets de cuivre et de zinc peuvent être déterminés de deux façons:

- d'après l'évaluation de la perte de matière mesurée par courants de Foucault dans le cadre des 
Fig. 25 Les contrôles effectués sur les effluents chimiques liquides sont fixés dans les décisions modalités et limites de chaque site. Ils portent sur les capacités de collecte, les émissaires de différents équipements et l'ouvrage de rejet principal, comme l'indique le schéma de principe ci-dessous.

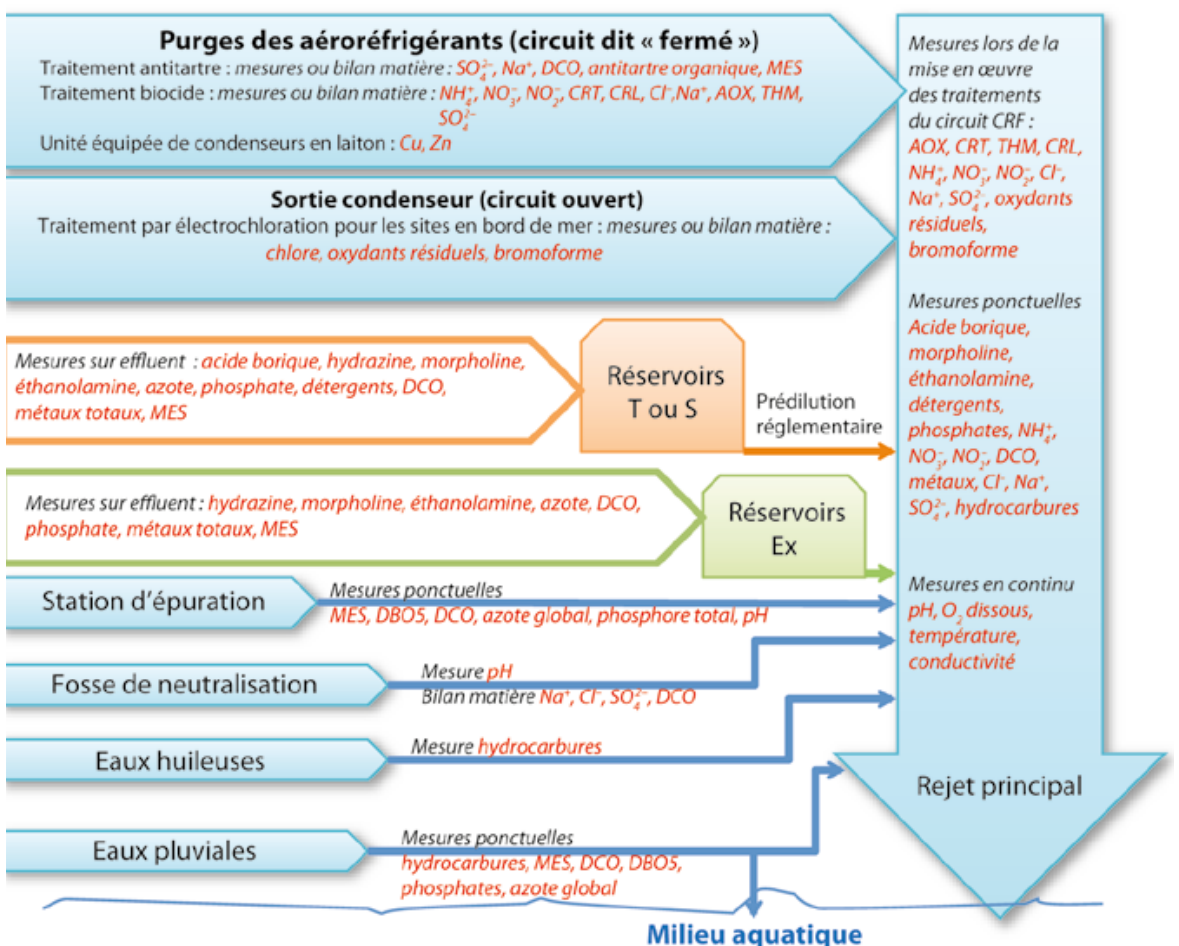

programmes de maintenance. Ces contrôles permettent d'estimer la perte d'épaisseur des tubes sur la durée de fonctionnement ;

- à partir de mesures chimiques pratiquées dans le rejet général et à l'amont afin de déterminer les valeurs ajoutées (déduction faite des métaux déjà présents à l'amont).

\section{Contrôle des rejets par bilan matière}

Lorsqu'il n'est pas possible de mesurer les substances chimiques dans l'effluent en particulier si les concentrations y sont trop faibles, le rejet est déterminé à partir des quantités de produits injectés dans les circuits et de la connaissance des transformations de ces produits lors de la réaction chimique. Cette pratique est notamment utilisée pour les rejets de substances issues de la station de production d'eau déminéralisée (sodium, chlorures, sulfates) ou pour certains rejets des traitements antitartre et biocides.

\section{Contrôle dans le milieu aquatique en aval du rejet principal}

Des mesures sont pratiquées dans le milieu récepteur en aval des rejets :

- périodiquement sur des substances faisant l'objet de limites à ne pas dépasser;

- en continu par la station multiparamètres aval $(\mathrm{pH}$, $\mathrm{O}_{2}$ dissous, conductivité, $\mathrm{T}^{\circ}$ ).

Sur les sites en bord de rivière, des mesures sont aussi réalisées ponctuellement ou en continu en amont du site (MES, métaux...) pour tenir compte de l'éventuelle présence de ces substances dans le calcul des rejets « ajoutés » dans l'ouvrage principal.

\section{Limites de rejet}

Les limites à ne pas dépasser dans les rejets pour les substances chimiques sont fixées par les décisions limites accordées à chaque centrale nucléaire par l'ASN (cf. chapitre 5 sur la réglementation). Ces limites peuvent porter sur:

- les concentrations maximales ajoutées dans les émissaires de rejet ;

- les concentrations maximales ajoutées dans l'ouvrage principal de rejet ;

- les flux de rejet annuels, mensuels, journaliers, et/ ou sur 2 heures (flux ajoutés);

- les concentrations ajoutées dans le milieu aquatique en aval des rejets.

Le choix des substances réglementées est fait sur la base des informations relatives aux rejets et à leur impact sur l'environnement et la santé présentées dans le dossier de demande d'autorisation (cf. chapitre 9).

Par ailleurs, les effluents rejetés ne doivent dégager aucune odeur, ni provoquer une quelconque coloration de l'eau.

Compte tenu des différences de conception entre les centrales, s'agissant des circuits non nucléaires et de la particularité liée à l'environnement de chaque site, les limites autorisées par la réglementation diffèrent d'un site à l'autre. À titre d'exemple, l'encart ci-après fournit les limites chimiques fixées pour la centrale nucléaire de Saint-Laurent-des-Eaux. 
Extrait de l'arrêté du 19 mars 2015 portant homologation de la décision no 2015-DC-0498 de l'Autorité de sûreté nucléaire du 19 février 2015 fixant les limites de rejets dans I'environnement des effluents du CNPE de Saint-Laurent-desEaux (2 unités de $900 \mathrm{MWe}$ )

a) Ouvrage de rejet principal :

Les limites en concentration se calculent par différence entre la concentration mesurée ou calculée à l'ouvrage de rejet principal et la concentration mesurée en amont corrigée afin de prendre en compte le phénoméne d'évaporation des eaux pompées dans les aéroréfrigérants.

\begin{tabular}{|c|c|c|c|c|c|}
\hline SUBSTANCES & $\begin{array}{l}\text { PRINCIPALES } \\
\text { ORIGINES }\end{array}$ & $\begin{array}{l}\text { FLUX } 2 \text { H } \\
\text { AJOUTÉ } \\
(\mathrm{kg})\end{array}$ & $\begin{array}{l}\text { FLUX } 24 \mathrm{H} \\
\text { AJOUTÉ } \\
(\mathrm{kg})\end{array}$ & $\begin{array}{l}\text { FLUX } \\
\text { ANNUEL } \\
\text { AJOUTÉ } \\
(\mathrm{kg})\end{array}$ & $\begin{array}{l}\text { CONCENTRATION } \\
\text { MAXIMALE AJOUTÉE } \\
\text { DANS L'OUVRAGE } \\
\text { DE REJET PRINCIPAL } \\
(\mathrm{mg} / \mathrm{L})\end{array}$ \\
\hline Acide borique (1) & Réservoirs T, S & 250 & 1400 & 10000 & 29 \\
\hline Morpholine (1) & $\begin{array}{l}\text { Réservoirs T, S et Ex } \\
\text { Réseau SEO }\end{array}$ & - & 11 & 500 & 0,71 \\
\hline Ethanolamine (2) & $\begin{array}{l}\text { Réservoirs T, S et Ex } \\
\text { Réseau SEO }\end{array}$ & - & $9,5(3)$ & 400 & 1,7 \\
\hline Hydrazine & Réservoirs T, S et Ex & - & $1,5(4)$ & 16 & 0,10 \\
\hline $\begin{array}{l}\text { Azote (ammonium, } \\
\text { nitrites, nitrates }\end{array}$ & Réservoirs T, $\mathrm{S}$ et $\mathrm{Ex}$ & - & 54 & 6000 & \multirow{4}{*}{$3,5(5)$} \\
\hline Ammonium & \multirow{3}{*}{$\begin{array}{l}\text { Traitement à la } \\
\text { monochloramine }\end{array}$} & - & 70 & - & \\
\hline Nitrates & & - & $1470(6)$ & - & \\
\hline Nitrites & & - & $70(6)$ & - & \\
\hline Phosphates & $\begin{array}{l}\text { Réservoirs T, S et Ex } \\
\text { Réseau SEO }\end{array}$ & 10 & 90 & 710 & 1,7 \\
\hline Détergents & Réservoirs T, $\mathrm{S}$ & 30 & 100 & 1500 & 3,5 \\
\hline $\begin{array}{l}\text { Métaux totaux } \\
\text { (cuivre, zinc, man- } \\
\text { ganèse, nickel } \\
\text { titane, chrome, fer } \\
\text { aluminium (8) }\end{array}$ & Réservoirs $\mathrm{T}$, $\mathrm{S}$ et $\mathrm{Ex}$ & - & - & $62(9)$ & 0,14 \\
\hline DCO & Réservoirs T, $\mathrm{S}$ et Ex & - & 165 & - & 1,7 \\
\hline MES & $\begin{array}{l}\text { Réservoirs T, S et Ex } \\
\text { Station de } \\
\text { déminéralisation }\end{array}$ & - & 80 & - & 5,3 \\
\hline \multirow[b]{2}{*}{ Chlorures (10) } & $\begin{array}{l}\text { Station de } \\
\text { déminéralisation }\end{array}$ & \multirow[b]{2}{*}{-} & \multirow[b]{2}{*}{1740} & \multirow[b]{2}{*}{-} & \multirow[b]{2}{*}{14} \\
\hline & $\begin{array}{l}\text { Traitement à la } \\
\text { monochloramine } \\
\text { Chloration massive }\end{array}$ & & & & \\
\hline \multirow{3}{*}{ Sodium (11) } & Réservoirs T, S et Ex & \multirow{3}{*}{-} & \multirow{3}{*}{1900} & \multirow{3}{*}{-} & \multirow{3}{*}{20} \\
\hline & $\begin{array}{l}\text { Station de } \\
\text { déminéralisation }\end{array}$ & & & & \\
\hline & $\begin{array}{l}\text { Traitement à la } \\
\text { monochloramine } \\
\text { Chloration massive }\end{array}$ & & & & \\
\hline $\begin{array}{l}\text { Chlore résiduel total } \\
\text { (CRT) }\end{array}$ & $\begin{array}{l}\text { Traitement à la } \\
\text { monochloramine } \\
\text { Chloration massive }\end{array}$ & - & $45(12)$ & 4500 & 0,31 \\
\hline AOX & $\begin{array}{l}\text { Traitement à la } \\
\text { monochloramine } \\
\text { Chloration massive }\end{array}$ & - & $15(13)$ & $1000(14)$ & 0,11 \\
\hline THM & Chloration massive & 2,5 & 9,5 & - & 0,3 \\
\hline \multirow[t]{2}{*}{ Sulfates (15) } & $\begin{array}{l}\text { Station de } \\
\text { déminéralisation }\end{array}$ & \multirow[t]{2}{*}{-} & \multirow[t]{2}{*}{1925} & \multirow[t]{2}{*}{-} & \multirow[t]{2}{*}{41} \\
\hline & Chloration massive & & & & \\
\hline
\end{tabular}


(1) Lors d'une vidange complète ou partielle d'un réservoir d'acide borique (réservoir REA bore ou PTR), les limites des flux $2 \mathrm{~h}$, $24 \mathrm{~h}$ et annuel sont portées respectivement à $500 \mathrm{~kg}, 1900 \mathrm{~kg}$ et $13000 \mathrm{~kg}$. La concentration maximale ajoutée au rejet est portée à 59 mg/L. Cette vidange ne peut être pratiquée qu'après démonstration que ces réservoirs ne peuvent être ramenés dans le cadre des spécifications. (2) En cas de changement du conditionnement du circuit secondaire, les limites du flux $24 \mathrm{~h}$ de l'ancien conditionnement restent applicables jusqu'à la fin de cycle des deux réacteurs Dans les cas où les deux modes de conditionnement du circuit secondaire (morpholine ou éthanolamine) seraient utilisés durant la même année calendaire, les limites annuelles sont calculées:

- pour l'ancien conditionnement, au prorata temporis de la durée de fonctionnement jusqu'à la fin de cycle du dernier réacteur ;

- pour l'ancien conditionnement, au prorata temporis de la durée de fonctionnement à partir de la date de basculement.

(3) Sur l'année, $5 \%$ des flux 24 h peuvent dépasser cette valeur sans toutefois dépasser $23 \mathrm{~kg}$.

(4) Sur l'année, $2 \%$ des flux 24 heures peuvent dépasser 1,5 kg sans toutefois dépasser $2 \mathrm{~kg}$. Dans cette configuration, la concentration moyenne ajoutée en Loire est portée à 0,0005 mg/L.

(5) Les concentrations sont exprimées en azote.

(6) La limite du flux 24 heures est portée à $1840 \mathrm{~kg}$ en cas de traitement à la monochloramine renforcé.

(7) Lors de la période de traitement à la monochloramine, $10 \%$ des flux 24 heures peuvent dépasser $70 \mathrm{~kg}$ sans toutefois dépasser $230 \mathrm{~kg}$.

(8) Les flux annuels de chacun des métaux nickel et chrome n'excèdent pas $30 \%$ des rejets annuels de métaux totaux.

(9) Les flux annuels de chacun des métaux nickel et chrome n'excèdent pas $30 \%$ des rejets annuels de métaux totaux.

(10) Les limites du flux 24 h et de la concentration ajoutée dans l'ouvrage de rejet sont portées respectivement à :

$2140 \mathrm{~kg}$ et $17 \mathrm{mg} / \mathrm{L}$ en cas de traitement à la monochloramine renforcé ;

$2400 \mathrm{~kg}$ et $42 \mathrm{mg} / \mathrm{L}$ en cas de chloration massive.

(11) Les limites du flux 24 h et de la concentration ajoutée dans l'ouvrage de rejet sont portées respectivement à :

$2160 \mathrm{~kg}$ et $22 \mathrm{mg} / \mathrm{L}$ en cas de traitement à la monochloramine renforcé ;

$2330 \mathrm{~kg}$ et $43 \mathrm{mg} / \mathrm{L}$ en cas de chloration massive.

(12) La limite du flux 24 h est portée à $65 \mathrm{~kg}$ en cas de traitement à la monochloramine renforcé et à $100 \mathrm{~kg}$ en cas de chloration massive. Dans ces configurations, ajoutée dans l'ouvrage de rejet est portée à $2 \mathrm{mg} / \mathrm{l}$. Lorsqu'une chloration massive est réalisée quand le débit de la Loire est inférieur à $60 \mathrm{m3} / \mathrm{s}$, la concentration moyenne ajoutée en Loire est limitée à 0,019 mg/L.

(13) La limite du flux 24 h est portée à $20 \mathrm{~kg}$ en cas de traitement à la monochloramine renforcé et à $85 \mathrm{~kg}$ en cas de chloration massive. Dans ces configurations, la concentration ajoutée dans l'ouvrage de rejet est portée à 2,3 $\mathrm{mg} / \mathrm{L}$. Lorsqu'une chloration massive est réalisée quand le débit de la Loire est inférieur à $60 \mathrm{m3} / \mathrm{s}$, la concentration moyenne ajoutée en Loire est limitée à 0,016 mg/L.

(14) La limite du flux annuel d'AOX est augmentée de $75 \mathrm{~kg}$ par opération de chloration massive sans toutefois excéder $1220 \mathrm{~kg}$.

(15) Les limites du flux $24 \mathrm{~h}$ et de la concentration ajoutée dans l'ouvrage de rejet sont portées respectivement à $6950 \mathrm{~kg}$ et $195 \mathrm{mg} / \mathrm{L}$ en cas de chloration massive

b) Ouvrage secondaire de rejet en Loire :

Les effluents provenant du ruissellement des eaux pluviales doivent respecter, après traitement éventuel, une concentration limite de $5 \mathrm{mg} / \mathrm{L}$ en hydrocarbures.

c) Ouvrage SEO-SLA :

Les effluents provenant du ruissellement des eaux pluviales doivent respecter, après traitement éventuel, une concentration limite de $5 \mathrm{mg} / \mathrm{L}$ en hydrocarbures.

Niveau annuel des rejets chimiques liquides contrairement aux rejets radioactifs - assez homogènes -, les rejets annuels des principales substances chimiques sont très variables d'un site à l'autre (cf. tableau VIII), en raison notamment des différences de conception de certains circuits et des difficultés rencontrées du fait de la qualité d'eau brute utilisée pour les besoins du site (présence de silice, eau entartrante...).

\subsection{REJETS GAZEUX NON RADIOACTIFS}

\section{Rejets gazeux non radioactifs liés au conditionnement chimique du circuit secondaire}

Les produits de conditionnement chimique du circuit secondaire, à savoir l'ammoniaque, l'éthanolamine, la morpholine et l'hydrazine sont volatils et donc susceptibles d'être rejetés sous forme gazeuse. Les sources potentielles d'émissions de ces rejets gazeux sont les suivantes:

- le système GCTa et l'échappement de la turbopompe ASG dans les phases d'arrêt et de redémarrage de la tranche ;
- l'extraction du ventilateur des buées du CET ;

- la cheminée du BAN via DVN à laquelle le collecteur commun d'extraction d'air du système CVI est relié.

Ces rejets sont caractérisés dans les études d'impact.

Rejets gazeux non radioactifs liés au lessivage chimique des générateurs de vapeur

L'encrassement des générateurs de vapeur peut nécessiter un lessivage chimique à l'origine de rejets chimiques gazeux (ammoniac, dioxyde de carbone...). Ces rejets sont estimés et font l'objet d'une étude d'impact transmise à l'ASN dans le dossier de demande d'autorisation de l'opération. Selon le procédé de nettoyage mis en œuvre, des mesures de la concentration en ammoniac sont réalisées dans l'atmosphère.

\section{Rejets gazeux non radioactifs liés aux traitements biocides des circuits de refroidissement dits "fermés »}

Le traitement à la monochloramine engendre des rejets gazeux liés au phénomène de dégazage au niveau de la tour aéroréfrigérante, en particulier de monochloramine (CRT) et d'ammoniac.

Par ailleurs, les rejets chimiques gazeux résultant de l'injection d'hypochlorite de sodium lors d'une opé- 
Tableau VIIII le tableau ci-dessous présente l'ordre de grandeur des rejets annuels ramenés par unité de production pour les substances chimiques, en fonction de leur provenance.

\begin{tabular}{|c|c|c|}
\hline PROVENANCE & $\begin{array}{l}\text { SUBSTANCES } \\
\text { CHIMIQUES }\end{array}$ & $\begin{array}{l}\text { QUANTITÉ ANNUELLE REJETÉE } \\
\text { POUR UNE UNITÉ EN kg }\end{array}$ \\
\hline \multirow{7}{*}{$\begin{array}{c}\text { Substances chimiques associées } \\
\text { aux effluents radioactifs et aux eaux } \\
\text { d'exhaure des salles } \\
\text { des machines }\end{array}$} & Acide borique & 500 à 7000 \\
\hline & Hydrazine & $\leq 6$ \\
\hline & Lithine & $\leq 3$ \\
\hline & Morpholine & 20 à 350 \\
\hline & Éthanolamine & 2 à 20 \\
\hline & Ammonium & $\leq 7000$ \\
\hline & Phosphates & $\leq 700$ \\
\hline \multirow{2}{*}{$\begin{array}{c}\text { Usure des tubes } \\
\text { de condenseurs en laiton (Belleville, } \\
\text { Cattenom, Cruas, Dampierre) }\end{array}$} & Cuivre & $\leq 10500$ \\
\hline & Zinc & $\leq 4000$ \\
\hline \multirow{3}{*}{$\begin{array}{l}\text { Traitement antitartre } \\
\text { des aéroréfrigérants }\end{array}$} & $\begin{array}{c}\text { Sulfates } \\
\text { (Cruas, Cattenom, Golfech, } \\
\text { Nogent, Chooz) }\end{array}$ & 130000 à 6600000 \\
\hline & $\begin{array}{c}\text { Chlorures } \\
\text { (Cattenom jusqu'en 2016) }\end{array}$ & 530000 à 800000 \\
\hline & $\begin{array}{l}\text { Polyacrylates } \\
\text { (Bugey, Nogent) }\end{array}$ & 24000 à 120000 \\
\hline \multirow{8}{*}{$\begin{array}{c}\text { Traitement biocide } \\
\text { des circuits } \\
\text { de refroidissement dits « fermés » } \\
\text { à la monochloramine. } \\
\text { (Bugey, Dampierre, Chinon, Cruas, } \\
\text { St-Laurent, Cattenom, Golfech, Nogent, } \\
\text { Chooz) }\end{array}$} & Ammonium & $\leq 3500$ \\
\hline & Nitrates & 6200 à 100000 \\
\hline & Nitrites & $\leq 4000$ \\
\hline & AOX & $\leq 500$ \\
\hline & THM & 0 \\
\hline & Sodium & 6500 à 80000 \\
\hline & Chlorures & 10000 à 110000 \\
\hline & Chlore Résiduel Total & $\leq 2400$ \\
\hline $\begin{array}{l}\text { Traitement biocide à l'eau de Javel des } \\
\text { sites marins }\end{array}$ & Bromoformes & 2500 à 13000 \\
\hline \multirow{5}{*}{$\begin{array}{l}\text { Station d'épuration } \\
\text { STEP }\end{array}$} & Azote K & $\leq 1000$ \\
\hline & $\mathrm{DBO} 5$ & $\leq 2400$ \\
\hline & DCO & $\leq 5800$ \\
\hline & $P$ & $\leq 210$ \\
\hline & Matières en suspension & $\leq 4200$ \\
\hline \multirow{4}{*}{$\begin{array}{l}\text { Station de production d'eau } \\
\text { déminéralisée }\end{array}$} & Sulfates & $\leq 78000$ \\
\hline & Chlorures & 800 à 110000 \\
\hline & Sodium & 5200 à 81000 \\
\hline & Matières en suspension & 120 à 55000 \\
\hline
\end{tabular}

ration de chloration massive à $\mathrm{pH}$ contrôlé sont de plusieurs types:

- l'acide hypochloreux HClO : au pH de l'eau du CRF, l'hypochlorite de sodium s'hydrolyse instantanément dans l'eau en acide hypochloreux ( $\mathrm{HClO}$ ) et en hydroxyde de sodium ( $\mathrm{NaOH})$. De par ses propriétés de volatilité, l'acide hypochloreux ( $\mathrm{HClO})$ peut être dégazé dans la tour aéroréfrigérante ;

- les THM : la mise en œuvre de chloration massive sur CRF est génératrice de THM, dont une part importante peut être dégazée dans la tour aéroréfrigérante.

\section{Émissions des groupes électrogènes de secours}

Les groupes électrogènes de secours composés de moteurs diesel ou de turbine à combustion (TAC) fonctionnant au gasoil sont destinés uniquement à alimenter des systèmes de sécurité et/ou à prendre le relais de l'alimentation électrique principale en 
cas de défaillance de celle-ci. Ils ont donc un rôle majeur en termes de sûreté nucléaire. Les émissions des gaz de combustion (SOx, NOx) de ces matériels de petites puissances sont faibles sachant qu'ils ne fonctionnent que peu de temps (moins de $50 \mathrm{~h} / \mathrm{an}$ par diesel) lors des essais périodiques ou d'incidents (les émissions d'une TAC de $24 \mathrm{MWth}$ sont de l'ordre de $50 \mathrm{~kg} / \mathrm{h}$ pour les SOx et $100 \mathrm{~kg} / \mathrm{h}$ pour les NOx). Une évaluation annuelle de ces émissions est demandée par l'administration.

\section{Émissions de produits pouvant affecter la couche d'ozone stratosphérique}

Une centrale nucléaire est équipée de groupes frigorifiques pour assurer la production d'eau glacée permettant de réfrigérer les locaux techniques et administratifs. La grande majorité des groupes froids qui contenaient des fluides pouvant appauvrir la couche d'ozone stratosphérique ont été remplacés par des matériels contenant des fluides sans impact sur la couche d'ozone (essentiellement du R. 134a). Cependant ces nouveaux fluides de type HFC peuvent accroitre l'effet de serre.

\section{Émissions de produits pouvant accroître l'effet de serre}

Les principaux fluides utilisés en centrale et pouvant accroître l'effet de serre en cas d'émission dans l'environnement sont :

- les nouveaux fluides frigorigènes utilisés dans les groupes frigorifiques;

- l'hexafluorure de soufre utilisé dans les appareils de commutation électrique.

Les émissions annuelles de ces fluides pouvant accroître l'effet de serre étaient de l'ordre de $6000 \mathrm{~kg}$ pour l'ensemble des centrales en 2018. Ces émissions sont comptabilisées et déclarées suivant les modalités définies dans la réglementation, et des actions d'amélioration sont prises pour réduire progressivement ces rejets. Ces émissions converties en équivalent $\mathrm{CO}_{2}$ pour l'ensemble des centrales nucléaires du parc français correspondent à 2 jours de fonctionnement d'une seule centrale thermique de $1000 \mathrm{MW}$ en rejets de $\mathrm{CO}_{2}$ générés (coefficient ADEME).

\section{Rejets thermiques}

Dans une centrale nucléaire, le fluide " eau-vapeur » du circuit secondaire suit un cycle thermodynamique dit cycle de Carnot (cf. figure 26) au cours duquel il échange de l'énergie thermique avec deux sources de chaleur, l'une chaude, l'autre froide.

La source chaude est constituée par l'eau du circuit primaire chauffée par l'énergie nucléaire du réacteur ; selon les points du circuit, la température de l'eau primaire varie de $284^{\circ} \mathrm{C}$ à $323^{\circ} \mathrm{C}$. La haute pression régnant dans le circuit primaire (155 bars) permet à l'eau de rester en phase liquide. L'énergie thermique est extraite du circuit primaire par les générateurs de vapeur. La vapeur produite sous pression (58 à 77 bars) entraîne le groupe turbo-alternateur puis s'échappe de celui-ci pour se condenser au contact des tubes froids du condenseur. Le circuit assurant le refroidissement du condenseur (circuit tertiaire) constitue la source froide dont la température varie entre $\mathrm{O}^{\circ} \mathrm{C}$ et $30^{\circ} \mathrm{C}$ environ.

La source froide, nécessaire au fonctionnement, peut être apportée :

- soit directement par l'eau prélevée en rivière ou en mer dans un circuit ouvert ;

- soit indirectement par l'air ambiant au moyen d'un aéroréfrigérant dans un circuit dit «fermé » (cf. annexe 8.6).
Le rendement théorique d'un tel cycle (rapport $\eta$ entre l'énergie utilisable pour la production d'électricité et l'énergie fournie par le réacteur) dépend uniquement de la température, exprimée en Kelvin, des sources chaude et froide ; il est de 48 \% environ?. Du fait des pertes thermiques et mécaniques, le rendement réel n'est que de 32 à $33 \%$, ce qui signifie que l'énergie thermique à évacuer au condenseur par la source froide représente les $2 / 3$ de l'énergie fournie par le réacteur ou le double de l'énergie électrique produite par la centrale.

\subsection{CARACTÉRISTIQUES DES REJETS THERMIQUES EN CIRCUIT OUVERT}

Lorsque la centrale est située sur un cours d'eau à grand débit (Bugey 2-3, St-Alban, Tricastin sur le Rhône), en bord de mer (Gravelines, Paluel, Penly, Flamanville) ou sur un estuaire (Blayais), l'eau prélevée à l'aide de pompes de circulation passe dans les nombreux tubes du condenseur où elle s'échauffe avant d'être restituée intégralement au milieu aquatique (cf. figure 27 et tableau IX).

$$
\text { 1. } \boldsymbol{\eta}=\left(\mathrm{T}_{\text {source chaude }}^{\circ}-\mathrm{T}_{\text {sourceffride }}^{\circ}\right) / \mathrm{T}_{\text {source chaude }}^{\circ} ;(300-25) / 573 \mathrm{~K}=0,48 \text {. }
$$


Fig. 26 La figure présente le principe du cycle eau-vapeur dans une centrale nucléaire. L'énergie thermique du circuit primaire est extraite par les générateurs de vapeur. La vapeur entraîne le groupe turboalternateur pour produire l'électricité puis elle est condensée. Le circuit de refroidissement tertiaire assure le refroidissement du condenseur.

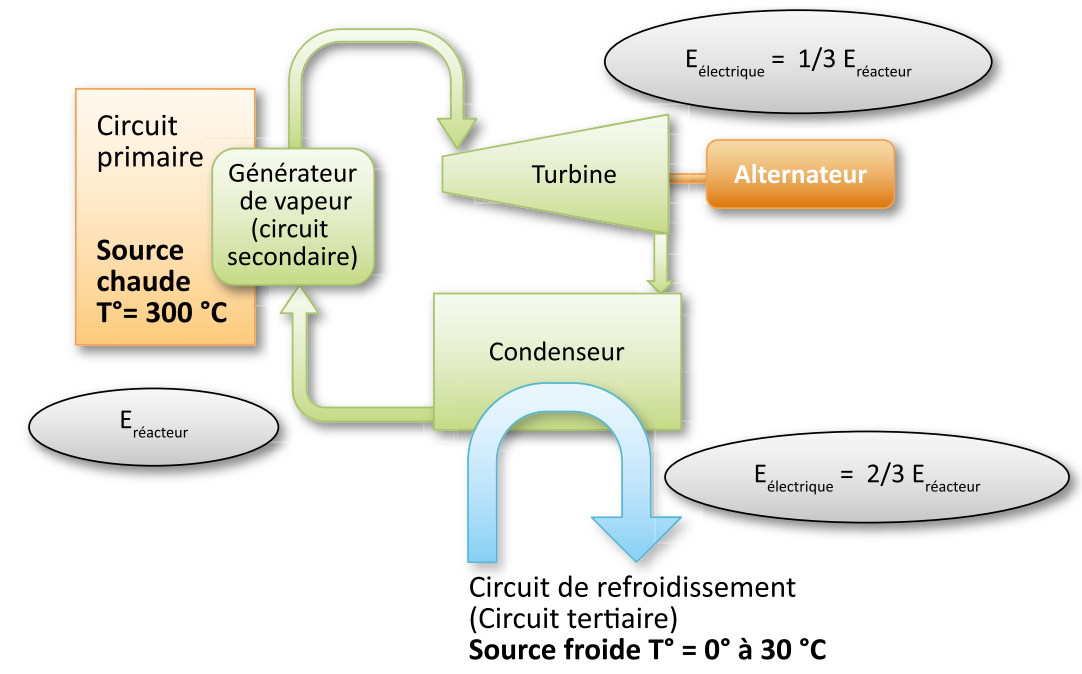

Fig. 27 Selon le schéma de principe présenté ci-dessous, en circuit ouvert,

le refroidissement du condenseur est réalisé en pompant l'eau du milieu naturel ; celle-ci s'échauffe avant d'être restituée au milieu naturel.

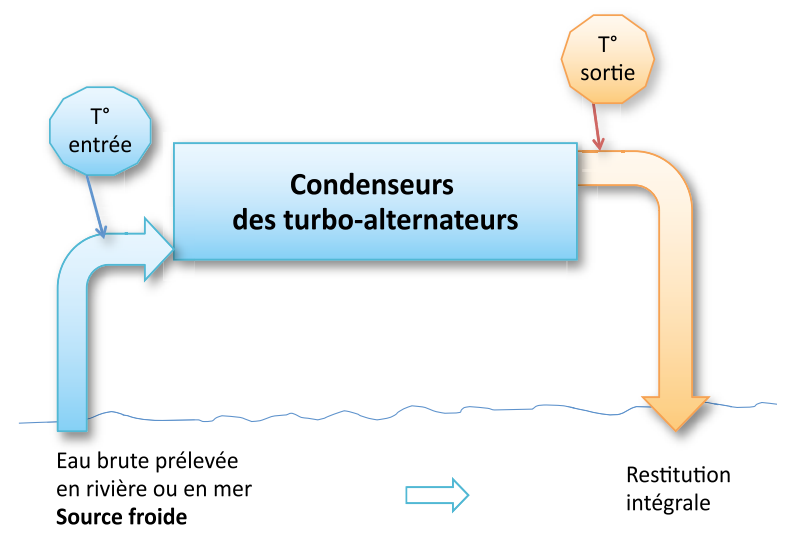

L'échauffement de l'eau (écart de température entre la sortie et l'entrée : $\Delta \mathrm{T}\left({ }^{\circ} \mathrm{C}\right)$ est lié à la puissance thermique $\left(P_{\text {th }}\right)$ à évacuer au condenseur et au débit d'eau brute au condenseur (Q) par la relation : $\Delta T^{1}=\mathrm{P}_{\text {th condenseur }} / \mathrm{Q}$.C. La connaissance du rendement thermique $(\eta)$ de l'installation permet d'exprimer l'échauffement en fonction de la puissance électrique produite par l'alternateur $\Delta \mathrm{T}=\mathrm{P}_{\text {électrique }} / \mathrm{Q} . \mathrm{C} . \eta$. Signalons, par ailleurs qu'en bord de mer, le débit d'eau de circulation dans les condenseurs varie de $10 \%$ à $15 \%$ avec la marée.

Les ouvrages de rejet sont conçus pour minimiser l'impact des rejets de chaleur sur l'écosystème (cf. chapitre 9 sur la maîtrise des impacts).
Tableau IX L'échauffement de l'eau dans le condenseur en fonctionnement nominal $\left(\mathrm{T}^{\circ}{ }_{\text {sortie }}-\mathrm{T}^{\circ}{ }_{\text {entrée }}\right)$ est de l'ordre de $10^{\circ} \mathrm{C}$ en rivière et de 10 à $15^{\circ} \mathrm{C}$ en mer.

\begin{tabular}{|c|c|c|c|c|}
\multicolumn{2}{c|}{ PALIER } & \multicolumn{2}{c|}{ PALIER } & \multicolumn{2}{c|}{ EPR } \\
$900 \mathrm{MWe}$ & \multicolumn{2}{|c|}{$1300 \mathrm{MWe}$} & $1650 \mathrm{MWe}$ \\
\hline Rivière & Mer & Rivière & Mer & Mer \\
\hline $10^{\circ} \mathrm{C}$ & $10-12^{\circ} \mathrm{C}$ & $10^{\circ} \mathrm{C}$ & $12-15^{\circ} \mathrm{C}$ & $12-14^{\circ} \mathrm{C}$ \\
\hline
\end{tabular}

\subsection{CARACTÉRISTIQUES DES REJETS THERMIQUES EN CIRCUIT DIT « FERMÉ »}

Afin de réduire le volume d'eau prélevée et limiter l'échauffement du milieu aquatique, le refroidissement des centrales comportant plusieurs unités de production implantées sur des cours d'eau à faible ou moyen débit est assuré en circuit dit «fermé » au moyen d'aéroréfrigérants (cf. figure 28).

Dans un aéroréfrigérant, une grande part de la chaleur extraite du condenseur (Q) est transférée directement à l'atmosphère (Q'> 0,9 Q) sous forme de chaleur latente de vaporisation (75\%) et sous forme de chaleur sensible (25\%).

Le reste de la chaleur est rejeté au cours d'eau par la purge (q). La purge de l'aéroréfrigérant constitue donc le rejet thermique de l'installation.

La température de l'eau de la purge répond à la relation : $\mathrm{T}_{\text {purge }}^{\circ}=\mathrm{T}_{\text {ha }}^{\circ}+\mathrm{A}$, où $\mathrm{T}_{\text {ha }}^{\circ}$ est la température humide de l'air et A l' " approche » qui est un paramètre de dimensionnement de l'aéroréfrigérant fixé à la conception (cf. figure 29). Pour les aéroréfrigérants des centrales nucléaires d'EDF, l'approche est de l'ordre de $11^{\circ} \mathrm{C}$. 
Fig. 28 Selon le schéma de principe ci-dessous, en circuit dit « fermé », le refroidissement du condenseur est réalisé au travers d'un circuit tertiaire muni de tour(s) aéroréfrigérante(s). Ces tours permettent de réduire la quantité d'eau prélevée et de limiter l'échauffement du milieu naturel lorsque les centrales sont implantées sur des rivières présentant des débits moyens ou faibles.

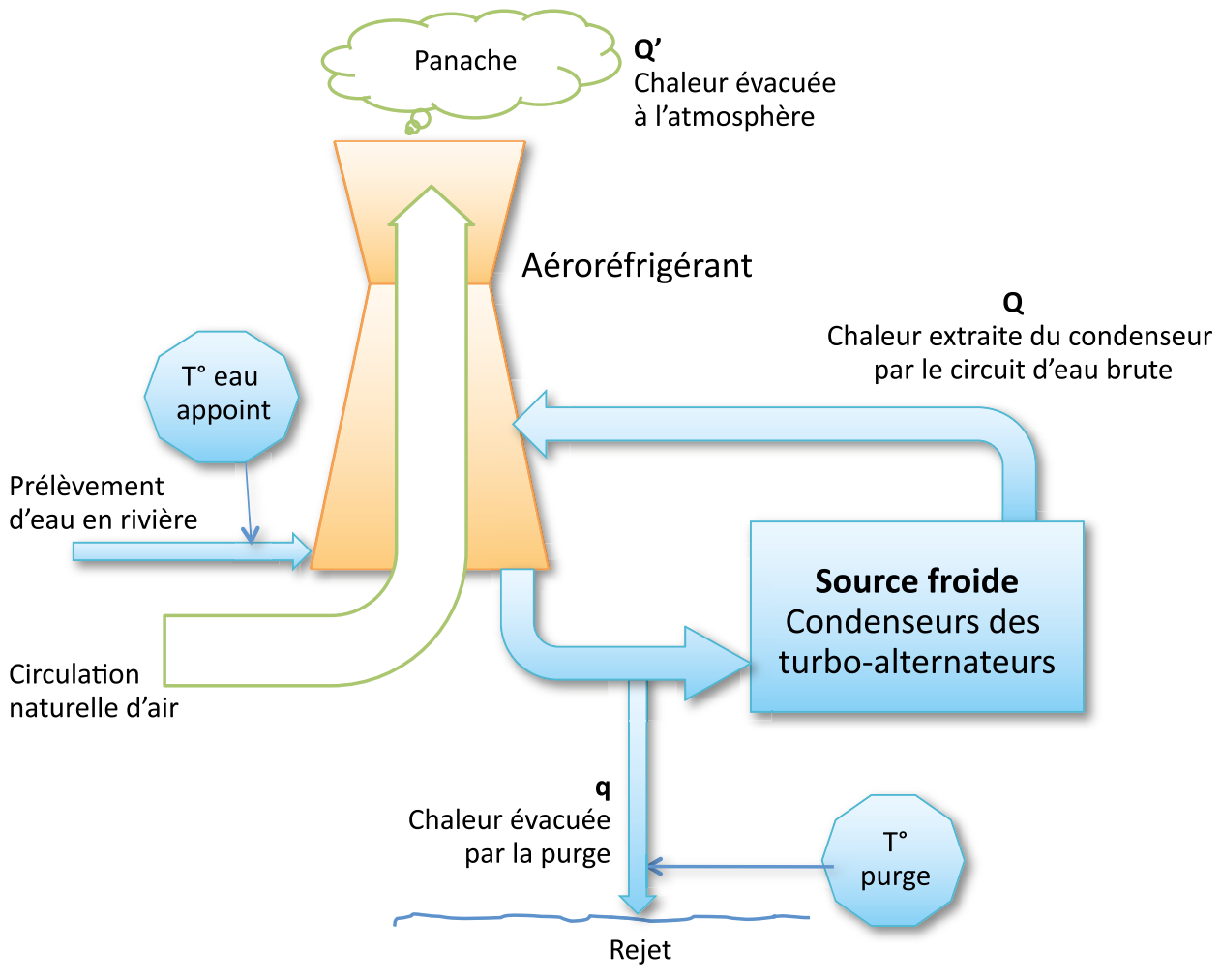

Fig. 29 La température de l'eau à la purge d'un aéroréfrigérant dépend des conditions atmosphériques, et notamment de la température et de l'humidité de l'air ambiant.

Température de la purge en ${ }^{\circ} \mathrm{C}$

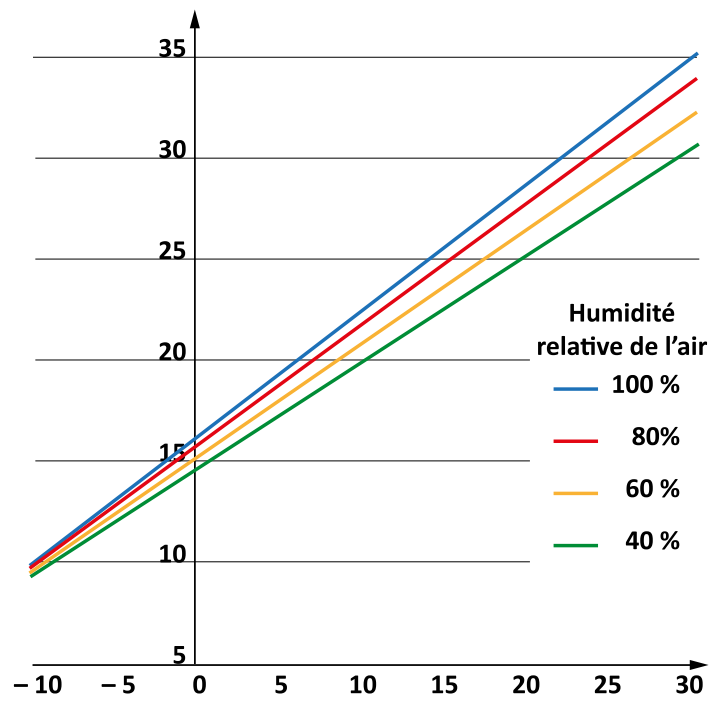

Température de l'air ambiant en ${ }^{\circ} \mathrm{C}$

Lorsqu'en été par exemple, la température de l'air chute brutalement à $12{ }^{\circ} \mathrm{C}$, alors que la température de l'eau du cours d'eau (qui est environ égale à la moyenne des températures des 15 derniers jours) reste à $25^{\circ} \mathrm{C}$, la température de l'eau de purge peut être inférieure à celle du cours d'eau :

$$
\mathrm{T}_{\text {purge }}^{\circ}=12^{\circ} \mathrm{C}+11^{\circ} \mathrm{C}=23^{\circ} \mathrm{C} \text {. }
$$

Dans le cas général, la température de la purge est supérieure à celle du cours d'eau. Cet écart est plus marqué en hiver qu'en été. Ainsi, si l'air hivernal devient subitement doux $\left(10^{\circ} \mathrm{C}\right)$ alors que la température du cours d'eau est basse $\left(1^{\circ} \mathrm{C}\right.$ ou $\left.2{ }^{\circ} \mathrm{C}\right)$, l'écart de température entre la purge et la prise d'eau peut atteindre une vingtaine de degrés (cf. chapitre 2 §3.4).

Le fonctionnement d'un aéroréfrigérant conduit " naturellement » à réduire voire à annuler le rejet thermique dû à la purge durant les périodes chaudes, ce qui est favorable à l'écosystème aquatique. L'efficacité des aéroréfrigérants dépend également de la propreté des installations, d'où la nécessité d'éviter les dépôts de tartre et de matières organiques sur les corps d'échanges (packing).

\subsection{REJETS THERMIQUES}

Respect des valeurs limites relatives aux rejets thermiques

Les contrôles destinés à s'assurer du respect des limites réglementaires s'appuient sur des mesures de température réalisées dans le rejet et dans l'en- 
vironnement ou sur des calculs effectués à partir de paramètres physiques tels que le rendement thermodynamique, l'énergie électrique produite, les débits de rejet et du cours d'eau (cf. encart ci-dessous).

\section{Limites de rejet}

Au niveau européen, les rejets thermiques ont été réglementés pour la première fois par la directive n 78/659/CEE du 18 juillet 1978 (aujourd'hui abrogée), puis par la directive n²006/44/CE du 6 septembre 2006 concernant la qualité des eaux douces ayant besoin d'être protégées ou améliorées pour être aptes à la vie des poissons (cf. chapitre 5 sur la réglementation). La directive de 2006 (aujourd'hui abrogée) fixait les limites suivantes:

- $\mathrm{T}^{\circ}$ aval $<28^{\circ} \mathrm{C}$; échauffement $<3^{\circ} \mathrm{C}$ pour les eaux cyprinicoles;

- $\mathrm{T}^{\circ}$ aval $<21,5{ }^{\circ} \mathrm{C}$; échauffement $<1,5^{\circ} \mathrm{C}$ pour les eaux salmonicoles.
La directive précisait que ces valeurs pouvaient être dépassées pendant $2 \%$ du temps (soit 7,3 jours par an) et des dérogations limitées géographiquement pouvaient être accordées par les États-membres dans des conditions particulières, et s'il était prouvé que ces dérogations n'avaient pas de conséquences nuisibles pour le développement équilibré des peuplements de poissons.

\section{Sites fluviaux}

Ces valeurs limites sont reprises par l'arrêté INB (article 4.1 .2 -II) qui renvoie à l'article 31 de l'arrêté du 2 février 1998 relatif aux prélèvements et à la consommation d'eau ainsi qu'aux émissions de toute nature des ICPE soumises à autorisation.

\section{Valeurs limites relatives aux rejets thermiques}

\section{Centrales implantées sur des cours d'eau}

Les valeurs limites relatives aux rejets thermiques sont vérifiées à partir de mesures de température et de paramètres de fonctionnement de la centrale. Ces données permettent de suivre :

- l'échauffement du cours d'eau entre l'amont et l'aval après mélange, noté : $\Delta \mathbf{T}^{\circ}$;

- la température aval après mélange, notée $: \mathbf{T}^{\circ}$

- la température du rejet, le cas échéant $: \mathrm{T}^{\circ}{ }_{\text {rejet }}$

a) Les mesures de température en amont et en aval après mélange

Dans ce cas, le $\Delta T^{\circ}$ est calculé par la formule [1] et les $\mathrm{T}^{\circ}{ }_{\text {amont }}$ et $\mathrm{T}^{\circ}$ aval après mélange sont données par la mesure.

[1] $\Delta T^{\circ}=T_{\text {aval }}^{\circ}-T_{\text {amont }}^{\circ}$; cas de la centrale de Nogent refroidie en circuit dit «fermé ».

b) Lorsque les mesures ne permettent pas de connaître avec précision la température " aval après mélange ", $\Delta T^{\circ}$ et $T_{\text {aval aprèsmélange }}^{\circ}$ sont calculés à partir des formules [2], [3] et [4] en fonction du débit du rejet principal $\left(Q_{\text {rejer }}\right)$, du débit du cours d'eau en aval $\left(Q_{\text {rivière }}\right)$ et de la puissance de la centrale $\left(P_{\text {thermique }}\right)$.

[2] $\Delta \boldsymbol{T}^{\circ}=\left(\boldsymbol{T}_{\text {rejet }}^{\circ}-\boldsymbol{T}_{\text {rivière amont }}^{\circ} \times \mathbf{Q}_{\text {rejet }} / \mathbf{Q}_{\text {rivière }} ;\right.$ cas des centrales de Golfech, Dampierre, St-Laurent et Chinon, Belleville, Chooz, Civaux refroidies en circuit dit «fermé ». Les formules de calcul sont semblables pour Cattenom et Cruas (cf. décisions modalités des sites).

[3] $\Delta T^{\circ}=\left(P_{\text {site }} \times\right.$ rendement thermique $) /\left(Q_{\text {canal de Donzère }} \times\right.$ chaleur massique de l'eau $)$ à Tricastin,

$[4] \Delta T^{\circ}=\left(P_{\text {thermique }}\right) /\left(Q_{\text {rivière }} \times\right.$ chaleur massique de l'eau $)$ à St-Alban et Bugey.

Connaissant l'échauffement $\Delta T$, la $T_{\text {aval après mélange }}^{\circ}$ est déduite de la température « amont » par la formule [5].

[5] $T_{\text {aval après mélange }}^{\circ}=T_{\text {amont }}^{\circ}+\Delta T^{\circ}$

\section{Centrales en bord de mer et en estuaire (refroidies en circuit ouvert)}

Les valeurs limites relatives aux rejets thermiques sont vérifiées par :

- la mesure en continu de la température à l'entrée du condenseur (prise d'eau) pour Flamanville, Paluel, Penly et Gravelines, ou, pour Blayais, de la température de la Gironde en amont des prises d'eau,

- la mesure en continu dans le canal de rejet ou dans le puits ou bassin de rejet pour Paluel, Gravelines et Blayais, ou un calcul dans les bassins de rejets pour Flamanville et Penly.

Ces mesures permettent de calculer l'écart de température (échauffement ou $\Delta T$ ) entre le rejet et l'amont (prise d'eau en mer ou Gironde pour Blayais).

[7] $\Delta \boldsymbol{T}^{\circ}=\boldsymbol{T}_{\text {rejet }}^{\circ}-\boldsymbol{T}_{\text {prise d'eau }}^{\circ}$; cas des centrales de Gravelines, Paluel et Blayais.

Lorsqu'il n'est pas possible de connaître cet écart de température par des mesures, celui-ci est calculé en fonction de la puissance de l'installation et du débit de rejet.

[8] $\Delta \boldsymbol{T}^{\circ}=\boldsymbol{k}$. $\boldsymbol{P}_{\text {électrique brute }} / \mathbf{Q}_{\text {rejet }}$ (k étant un coefficient dépendant de la chaleur massique de l'eau et du rendement thermique de la centrale); cas des centrales de Penly et de Flamanville. 
Ainsi, la réglementation en vigueur relative aux rejets thermiques des centrales nucléaires doit être conforme aux limites fixées ci-dessus, sauf en cas de dispositions contraires fixées par l'ASN. Les limites relatives aux rejets thermiques spécifiques aux centrales nucléaires sont définies dans les décisions limites de chaque site (cf. annexe 8.7).

Les limites imposées aux centrales nucléaires françaises sont semblables à celles appliquées aux autres centrales européennes (cf. encart ci-contre).

\section{Conditions climatiques exceptionnelles}

À la suite de la canicule de 2003, des dispositions relatives aux conditions climatiques exceptionnelles ont été introduites dans les décisions limites de certains CNPE. Si des conditions climatiques exceptionnelles ne permettent pas de respecter les limites définies en conditions climatiques normales ET si le réseau de transport d'électricité (RTE) ou si l'équilibre entre la consommation et la production d'électricité requiert le fonctionnement de(s) la(les) centrale(s), les valeurs limites applicables aux rejets sont alors fixées selon :

- un delta de température entre l'amont et l'aval (échauffement) après mélange des effluents ;

- et/ou une température en aval après mélange à ne pas dépasser supérieure à la maximale autorisée en conditions climatiques normales, pouvant être relaxée de $+1{ }^{\circ} \mathrm{C}$ à $+2{ }^{\circ} \mathrm{C}$ selon les sites.

Les valeurs limites applicables aux rejets en conditions climatiques exceptionnelles sont prévues dans les décisions limites des sites concernés.

Les centrales possédant des exigences associées aux conditions climatiques exceptionnelles sont Bugey, Cattenom, Cruas, Golfech, Nogent, Saint-Alban et Tricastin.

\section{Situations exceptionnelles}

Si du fait d'une situation exceptionnelle (ex. canicule et/ou sécheresse), la poursuite du fonctionnement d'une INB nécessite une modification temporaire de certaines prescriptions (ex. en cas de températures en amont supérieures aux prescriptions des CNPE, seul un échauffement sera retenu) et si ce fonctionnement constitue une nécessité publique, un dossier au titre de l'article R. 593-40 alinéa II du Code de l'environnement sera déposé auprès de l'ASN et du ministère de ministère de la Transition écologique. Les consultations du CODERST et de la CLI ne seront pas exigées. Cependant, les centrales continueront d'informer leurs parties prenantes, comme cela est demandé pour les conditions climatiques exceptionnelles.

Les centrales qui pourraient être concernées par l'article R. 593-40 alinéa II du Code de l'environnement seraient Bugey, Cattenom, Cruas, Chooz, Golfech, Nogent, Saint-Alban et Tricastin.

\section{Exemples de limites thermiques en Europe}

La réglementation des rejets thermiques des pays européens est, comme en France, fondée sur la directive européenne de 1978 (aujourd'hui abrogée) et remplacée par la directive européenne n²006/44/CE. Les limites imposées aux centrales nucléaires européennes sont semblables à celles appliquées à nos installations, comme illustré ci-dessous pour les réacteurs en Belgique et au Royaume-Uni.

La réglementation applicable aux réacteurs belges de Tihange situés sur la Meuse et équipés de tours aéroréfrigérantes est la suivante :

- $\mathrm{T}^{\circ}{ }_{\text {aval }} \leq 28^{\circ} \mathrm{C}$ en moyenne tri-horaire $\left(29^{\circ} \mathrm{C}\right.$ avec dérogation $2 \%$ du temps) ;

- et $\mathrm{DT} \leq 4^{\circ} \mathrm{C}$ en moyenne journalière (dérogation $2 \%$ du temps entre le $01 / 07$ et le $31 / 03: \Delta \mathrm{T} \leq{ }^{\circ} \mathrm{C}$ si $\mathrm{Q}_{\text {Meuse }}<300 \mathrm{~m}^{3} / \mathrm{s}$ et $\mathrm{T}_{\text {amont }} \leq 21^{\circ} \mathrm{C}$ et $\Delta \mathrm{T}$ instantané $(\max 15 \mathrm{~min}) \leq 5^{\circ} \mathrm{C}$ ).

La température est mesurée en continu sur les eaux de refroidissement de ces réacteurs, aux points de déversement et dans le canal d'amenée. Par ailleurs, cette centrale est reliée au réseau régional de télésurveillance des eaux.

La réglementation applicable aux réacteurs belges de Doel situés dans l'estuaire de l'Escaut est la suivante, les réacteurs 1 et 2 étant en circuit ouvert et les réacteurs 3 et 4 étant en circuit dit «fermé »:

- $\mathrm{T}^{\circ}{ }_{\text {Rejet }} \leq 33^{\circ} \mathrm{C}$;

- $\mathrm{T}_{\text {Rejet }}^{\circ} \leq 32^{\circ} \mathrm{C}$ en moyenne journalière ;

- $\mathrm{T}^{\circ}{ }_{\text {Rejet }} \leq 30^{\circ} \mathrm{C}$ en moyenne sur 30 jours.

La température est mesurée en continu sur les eaux de refroidissement de ces réacteurs, au niveau de l'ouvrage général de rejet.

La réglementation applicable au réacteur britannique de Sizewell B, en circuit ouvert sur la mer du Nord est la suivante :

- l'échauffement ne doit pas dépasser celui équivalent à un rejet de $58 \mathrm{~m} 3 / \mathrm{s}$ à une température de $9,9^{\circ} \mathrm{C}$;

- la température au rejet doit être inférieure ou égale à $45^{\circ} \mathrm{C}$.

\section{Sites marins}

\section{Il n'existe pas de directive européenne limitant les} rejets thermiques pour les sites situés en bord de mer. En France, les valeurs limites indiquées dans l'arrêté INB (article 4.1.2 -II) renvoient à l'article 31 de l'arrêté du 2 février 1998 relatif aux prélèvements et à la consommation d'eau ainsi qu'aux émissions de toute nature des installations classées pour la protection de l'environnement soumises à autorisation qui indique une limite de température au rejet de $30^{\circ} \mathrm{C}$ et une élévation de température limitée à $2{ }^{\circ} \mathrm{C}$ pour les masses d'eau conchylicoles. 
Des limites spécifiques existent toutefois dans les décisions propres à chaque site marin.

\section{Niveau des rejets thermiques}

\section{Circuit ouvert en mer}

Les rejets thermiques en mer sont donnés à titre d'exemple pour la centrale de Gravelines qui rejette en mer du Nord (cf. tableau X).

\section{Circuit ouvert en rivière}

L'échauffement du canal de Donzère-Mondragon dû au fonctionnement de la centrale nucléaire de Tricastin refroidie en circuit ouvert est fourni au tableau XI.

\section{Circuit dit «fermé»}

L'échauffement des cours d'eau dû au fonctionnement d'une centrale nucléaire refroidie en circuit dit "fermé » est de quelques dixièmes de degrés (cf. tableau XII).

Le refroidissement en circuit dit «fermé » au moyen d'aéroréfrigérant permet de réduire le volume d'eau prélevé et l'échauffement du milieu aquatique par rapport à un circuit ouvert. À ce titre, ce procédé de refroidissement par aéroréfrigérants constitue, pour les centrales installées sur ce type de cours d'eau à débit moyen à faible, ce qu'il est convenu d'appeler la meilleure technique disponible (MTD) au sens réglementaire du terme (cf. chapitre 5 sur la réglementation).

Tableau X Le tableau suivant présente la température maximale journalière au rejet et l'échauffement moyen journalier de la centrale nucléaire de Gravelines (6 unités de $900 \mathrm{MWe}$ ).

\begin{tabular}{|c|c|c|c|}
\hline CENTRALE DE GRAVELINES & $\begin{array}{c}\text { VALEUR MOYENNE } \\
\text { SUR LA PÉRIODE } \\
2008-2017\end{array}$ & $\begin{array}{c}\text { VALEUR } \\
\text { MAXIMALE SUR } \\
\text { LA PÉRIODE } \\
\text { 2008-2017 }\end{array}$ & $\begin{array}{l}\text { LIMITE AUTORISÉE } \\
\text { SUR LA PÉRIODE } \\
\text { 2008-2017 }\end{array}$ \\
\hline $\begin{array}{l}\text { Température mesurée dans le canal de rejet } \\
d u 1^{\text {er }} \text { novembre au } 31 \text { mai (max. journalier) }\end{array}$ & $20,5^{\circ} \mathrm{C}$ & $27,2^{\circ} \mathrm{C}$ & $30^{\circ} \mathrm{C}$ \\
\hline 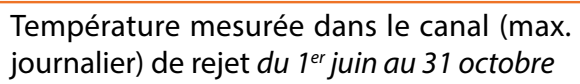 & $28,0^{\circ} \mathrm{C}$ & $33,1^{\circ} \mathrm{C}$ & $35^{\circ} \mathrm{C}$ \\
\hline $\begin{array}{l}\text { Échauffement entre le canal de rejet et la } \\
\text { prise d'eau (moy. journalière) }\end{array}$ & $9,5^{\circ} \mathrm{C}$ & $12^{\circ} \mathrm{C}$ & $12^{\circ} \mathrm{C}$ \\
\hline $\begin{array}{l}\text { Température mesurée en mer au thermo- } \\
\text { graphe } n^{\circ} 7 \text { (max. journalier) }\end{array}$ & $14,9^{\circ} \mathrm{C}$ & $26,5^{\circ} \mathrm{C}$ & $30^{\circ} \mathrm{C}$ \\
\hline
\end{tabular}

Tableau XI le tableau suivant présente l'échauffement moyen journalier du canal de Donzère-Mondragon dû au rejet de la centrale nucléaire de Tricastin et la température journalière en aval après mélange (4 unités de $900 \mathrm{MWe}$ ).

\begin{tabular}{|c|c|c|c|}
\hline CENTRALE DE TRICASTIN & $\begin{array}{c}\text { VALEUR } \\
\text { JOURNALIĖRE } \\
\text { MOYENNE MESURÉE } \\
\text { SUR LA PÉRIODE } \\
\text { 2008-2017 }\end{array}$ & $\begin{array}{l}\text { VALEUR } \\
\text { JOURNALIĖRE } \\
\text { MAXIMALE } \\
\text { MESURÉE SUR } \\
\text { LA PÉRIODE } \\
\text { 2008-2017 }\end{array}$ & $\begin{array}{l}\text { LIMITE AUTORISÉE } \\
\text { SUR LA PÉRIODE } \\
2008-2017 \\
\text { (EN CONDITIONS } \\
\text { CLIMATIQUES } \\
\text { NORMALES) }\end{array}$ \\
\hline $\begin{array}{l}\text { Échauffement amont - aval calculé } \\
\left(\Delta \mathrm{T}^{\circ} \text { après mélange }\right)\end{array}$ & $1,4^{\circ} \mathrm{C}$ & $5,5^{\circ} \mathrm{C}$ & $4^{\circ} \mathrm{C}-6^{\circ} \mathrm{C}^{*}$ \\
\hline $\begin{array}{l}\text { Température aval après mélange } \\
\text { calculée }\end{array}$ & $15,7^{\circ} \mathrm{C}$ & $27,8^{\circ} \mathrm{C}$ & $28^{\circ} \mathrm{C}$ \\
\hline
\end{tabular}

${ }^{*} \operatorname{si} Q_{\text {canal }}<480 \mathrm{~m}^{3} / \mathrm{s}$

Tableau XIl le tableau suivant présente l'échauffement moyen journalier du cours d'eau à Dampierre, St-Laurent et Chinon.

\begin{tabular}{|l|c|c|c|c|}
$\begin{array}{c}\text { SITES SUR LA LOIRE } \\
\text { (ÉCHAUFFEMENT } \\
\text { MOYEN JOURNALIER) }\end{array}$ & $\begin{array}{c}\text { PÉRIODE } \\
\text { ANALYSÉE }\end{array}$ & $\begin{array}{c}\text { VALEUR MOYENNE } \\
\text { CALCULÉE } \\
\text { SUR LA PÉRIODE } \\
\text { ANALYSÉE }\end{array}$ & $\begin{array}{c}\text { VALEUR MAXIMALE } \\
\text { CALCULÉE } \\
\text { SUR LA PÉRIODE } \\
\text { ANALYSÉE }\end{array}$ & $\begin{array}{c}\text { LIMITE } \\
\text { AUTORISÉE }\end{array}$ \\
\hline Dampierre & {$[2013-2017]$} & $0,09^{\circ} \mathrm{C}$ & $0,8{ }^{\circ} \mathrm{C}$ & $1{ }^{\circ} \mathrm{C}$ \\
\hline Belleville & {$[2012-2017]$} & $0,15^{\circ} \mathrm{C}$ & $0,7{ }^{\circ} \mathrm{C}$ & $1{ }^{\circ} \mathrm{C}$ \\
\hline Chinon & {$[2009-2018]$} & $0,13^{\circ} \mathrm{C}$ & $0,55^{\circ} \mathrm{C}$ & $1{ }^{\circ} \mathrm{C}$ \\
\hline
\end{tabular}




\section{Utilisation des eaux tièdes issues des circuits de refroidissement}

\subsection{OBJECTIF}

Dans une centrale refroidie en circuit dit "fermé » ou en circuit ouvert fonctionnant à pleine puissance, la température de l'eau tiède à la sortie des condenseurs est supérieure de quinze à vingt degrés à celle de l'air ambiant.

Cette source de chaleur peut être mise à profit pour alimenter des serres maraîchères ou horticoles, des fermes aquacoles, voire d'autres industries. Elle permet à ces installations de réaliser des économies d'énergie et limite le recours à des moyens de chauffage faisant appel à des énergies fossiles émettrices de gaz à effet de serre.

La fourniture gratuite d'eau tiède n'est garantie ni en température ni en débit. Elle peut être interrompue pour des raisons techniques (arrêt de la centrale). Le raccordement des installations bénéficiaires à plusieurs unités de la centrale améliore la continuité de la fourniture en eau. Pour maîtriser leur outil de production, les utilisateurs doivent disposer d'installations de secours.

\subsection{ASPECTS RÉGLEMENTAIRES ET APPLICATIONS}

La loi du 15 juillet 1980 sur les économies d'énergie et l'utilisation de la chaleur demandait aux exploitants de centrales électriques de contribuer à la production combinée d'électricité et de chaleur, notamment en favorisant, en accord avec les collectivités locales, la création et le développement de réseau de chaleur. Le décret n 81-542 du 13 mai 1981 précisait en particulier que la chaleur provenant des rejets thermiques est gratuite mais que les charges d'équipement, d'exploitation, d'entretien et de renouvellement des installations de récupération incombent à l'utilisateur. Ces dispositions sont désormais codifiées dans le Code de l'énergie. Les exigences relatives à la production de chaleur figurent désormais aux articles L. 711-1 à L. 711-3 et R. 711-1 à R. 711-4 du Code de l'énergie.

L'arrêté du 7 février 2012 modifié précise dans son article 4.1.4 que " tout transfert d'effluents liquides [...] à une autre installation, nucléaire de base ou non, dépendant d'un autre exploitant, fait préalablement l'objet d'une convention passée » entre le CNPE et l'autre installation. Cette convention fixe les caractéristiques et les quantités d'effluents ou des eaux transférées. Elle doit comporter également les obligations des deux exploitants en matière de contrôle et de surveillance.

Six centrales nucléaires valorisent la chaleur issue de l'eau sortant des condenseurs: Chinon, Civaux, Dampierre, Golfech, Gravelines et Saint-Laurent (cf. encart ci-dessous). Pour ces sites, il existe des conventions entre le producteur d'énergie thermique (les centrales) et l'exploitant du réseau de chaleur.

\section{Centrales fournissant leurs eaux tièdes}

\section{Saint-Laurent}

Le site de St-Laurent a servi de zone d'expérimentation et de démonstration des possibilités d'utilisation des eaux tièdes en aquaculture et en agriculture de 1975 à 1982. Une zone de 32 ha a été aménagée par une coopérative à usage agricole en 1984. Elle est alimentée par les eaux tièdes des deux unités de la centrale.

\section{Chinon}

La communauté de communes du Véron a créé une zone d'activité agro-industrielle de 40 ha, alimentée par les quatre unités de la centrale de Chinon $B\left(1 \mathrm{~m}^{3} / \mathrm{s}\right)$. Des entreprises horticoles et maraîchères s'y sont installées ainsi qu'une usine de séchage du bois en 1989.

\section{Dampierre}

La coopérative d'utilisation de matériels agricoles a signé une convention en 2009 avec la centrale pour alimenter un lotissement agricole de 120 ha (1 à $\left.2 \mathrm{~m}^{3} / \mathrm{s}\right)$. 


\section{Civaux}

Signée en 2001, une convention avec la commune de Civaux permet d'alimenter en eau tiède ( $\left.1000 \mathrm{~m}^{3} / \mathrm{h}\right)$ un élevage de crocodiles, une salle omnisport, une maison d'accueil pour personnes âgées et une piscine.

\section{Golfech}

Une convention a été signée en 1991 avec la commune de Golfech pour la fourniture de $300 \mathrm{~m}^{3} / \mathrm{h}$ d'eau destinée au chauffage d'une piscine, d'un groupe scolaire, d'une maison de retraite et d'une salle polyvalente.

\section{Gravelines}

\section{Centre aquacole}

Le centre aquacole de Gravelines, créé en 1983, a pris une dimension industrielle à partir de 1987. La société d'économie mixte Gravelines aquaculture, devenue Aquanord SA en 1988, a fait réaliser des travaux d'adduction d'eau qui permettent de prélever $18 \mathrm{~m}^{3} / \mathrm{s}$ d'eau tiède à la sortie de quatre des six unités de la centrale. Ce prélèvement alimente une écloserie marine (alevins de bars essentiellement) et une ferme de grossissement de bars et de daurades royales.

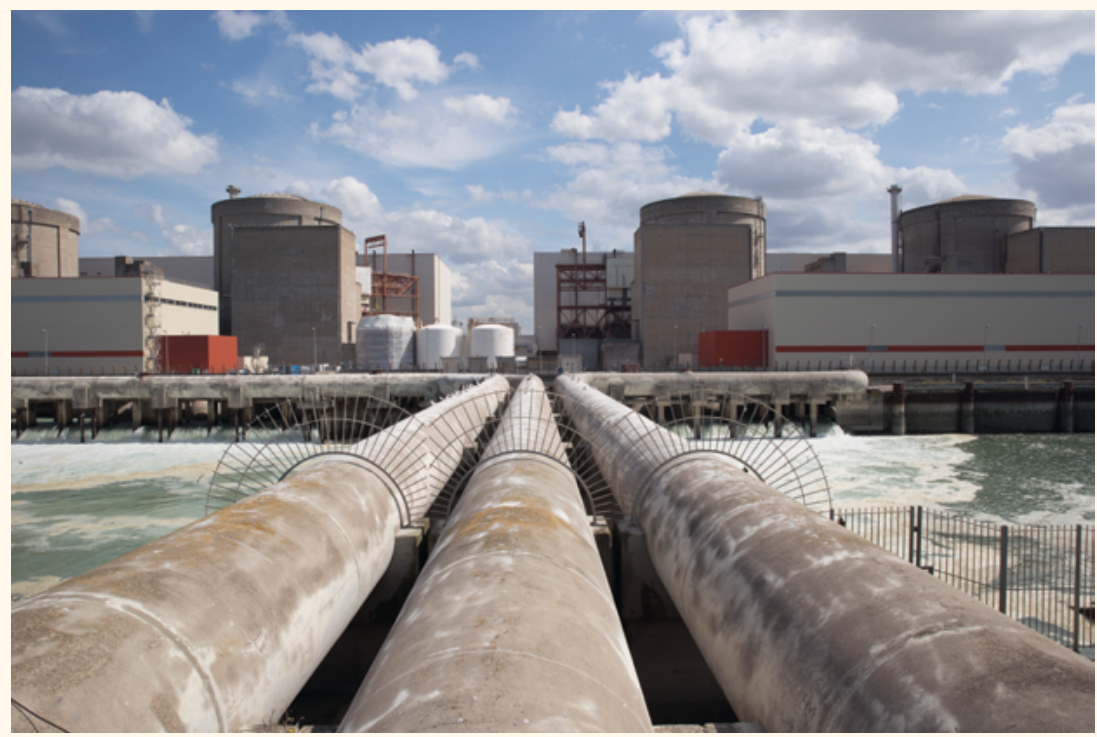

Conduites d'amenée d'eau tiède depuis le CNPE de Gravelines vers la ferme aquacole voisine (c) EDF - Bruno Conty

\section{Terminal méthanier}

Depuis 2015, l'eau tiède de la centrale nucléaire de Gravelines est utilisée pour réchauffer le gaz liquéfié du port méthanier. Cet apport d'énergie permet au port méthanier de se passer de la construction d'une centrale thermique au gaz de 250 MWth et donc d'éviter des émissions de gaz à effet de serre. Une convention a été signée en 2010 avec DK LNG pour fournir entre 8 et $12 \mathrm{~m}^{3} / \mathrm{s}$ d'eau tiède.

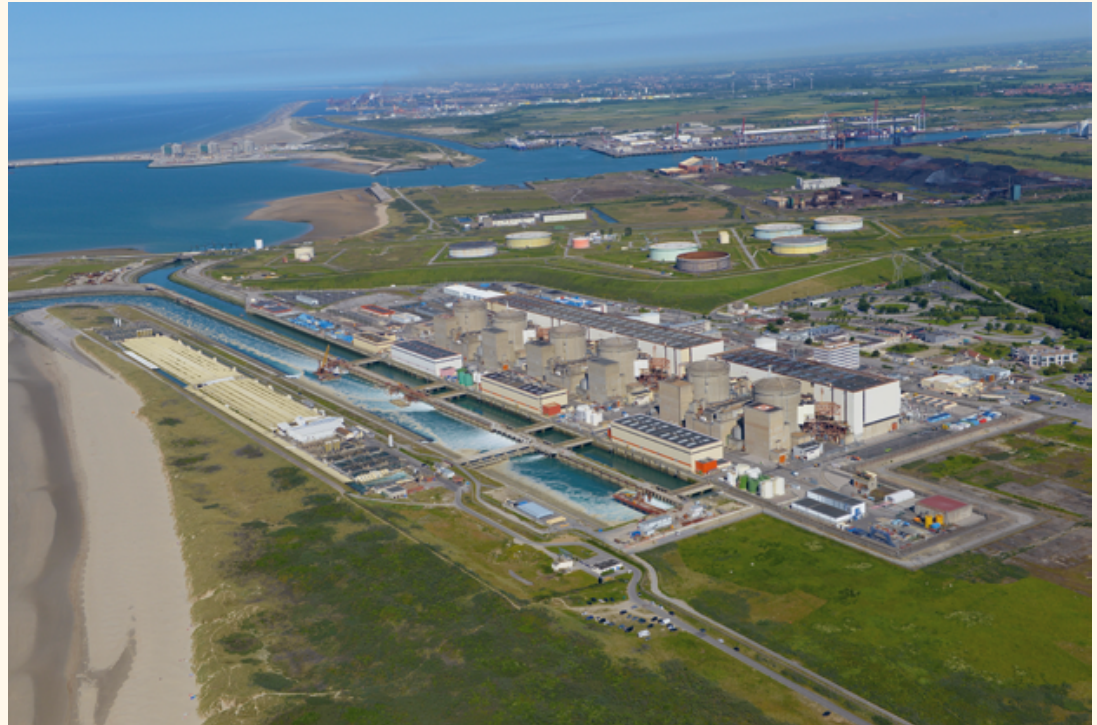

Vue aérienne de la centrale de Gravelines alimentant

en eaux tièdes la ferme aquacole (au premier plan) et le terminal méthanier de Dunkerque (arrière-plan à gauche) (c) EDF - Jean-Louis Burnod 


\section{Fonctionnement d'un réacteur à eau pressurisée}

La centrale nucléaire utilise l'énergie libérée par la fission nucléaire pour produire de l'électricité. Dans les réacteurs à eau sous pression (REP), le cœur du réacteur avec le combustible nucléaire est placé dans une cuve remplie d'eau. Cette eau, dont la température est d'environ $300{ }^{\circ} \mathrm{C}$, est maintenue sous forte pression par le pressuriseur (155 bars) pour éviter son ébullition ; elle est mise en circulation par des pompes dans un circuit fermé appelé « circuit primaire ». L'énergie thermique de l'eau primaire est transférée à un autre circuit d'eau dit « circuit secondaire » au moyen d'échangeurs de chaleur : les « générateurs de vapeur ». La vapeur issue des générateurs de vapeur actionne une turbine qui entraîne un alternateur produisant de l'électricité.

Les réacteurs nucléaires d'EDF en exploitation sont du type REP. Les réacteurs EPR (European Pressurized Reactor) sont du même type (cf. figure 30).

\section{Le combustible nucléaire}

Dans le cœur d'un réacteur de type REP, l'uranium se trouve sous forme de petites pastilles (environ $1 \mathrm{~cm}$ de diamètre et $1 \mathrm{~cm}$ de long) empilées dans un tube métallique étanche appelé gaine du combustible. Cette gaine métallique (en alliage de zirconium « zircaloy ») empêche les radionucléides de s'échapper dans l'eau du circuit primaire. Elle constitue la « première barrière » entre le combustible et l'environnement.

Ces tubes ou crayons sont assemblés de façon régulière dans une structure appelée assemblage combustible, qui regroupe quelques centaines de crayons (cf. figure 31). C'est entre ces tubes que circule l'eau primaire qui, au contact des crayons, va extraire la chaleur dégagée par la fission.

Fig. 30 Le schéma ci-dessous présente le principe d'un réacteur à eau pressurisée : l'eau du circuit primaire, maintenue à 155 bars et $300^{\circ} \mathrm{C}$ environ, transporte la chaleur produite dans la cuve du réacteur par les réactions de fission qui ont lieu à l'intérieur des crayons de combustible. L'énergie thermique de cette eau est transférée au circuit secondaire dans les générateurs de vapeur (GV). La vapeur produite dans les GV actionne un groupe turbo-alternateur pour produire l'électricité.

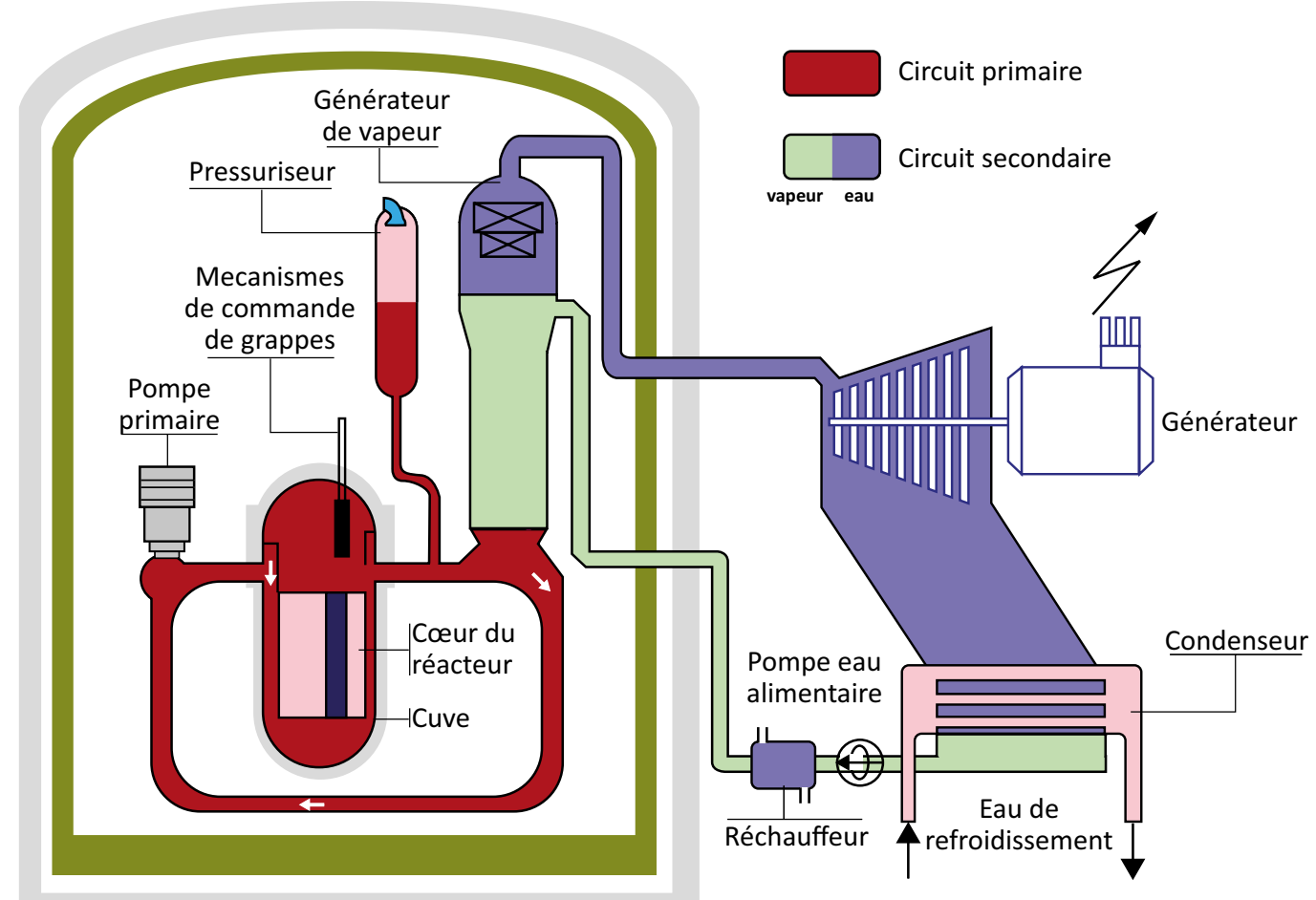


Dans ce type de réacteur, la réaction nucléaire en chaîne n'est possible dans la durée que si le combustible composé d'oxyde uranium est enrichi à hauteur de 3 à $5 \%$ en uranium 235 qui seul est fissile. L'uranium naturel n'en contient qu'environ 0,7 \% le reste étant de l'uranium 238. La fission peut aussi être obtenue sur des isotopes du plutonium. Certains combustibles sont donc réalisés à partir d'oxyde mixte d'uranium et de plutonium (appelé MOX).

Fig. 31 Les assemblages de combustible nucléaire neufs sont stockés dans une piscine après leur arrivée sur site, avant leur chargement dans le cœur du réacteur (๑ EDF - Damien CHARFEDDINE).

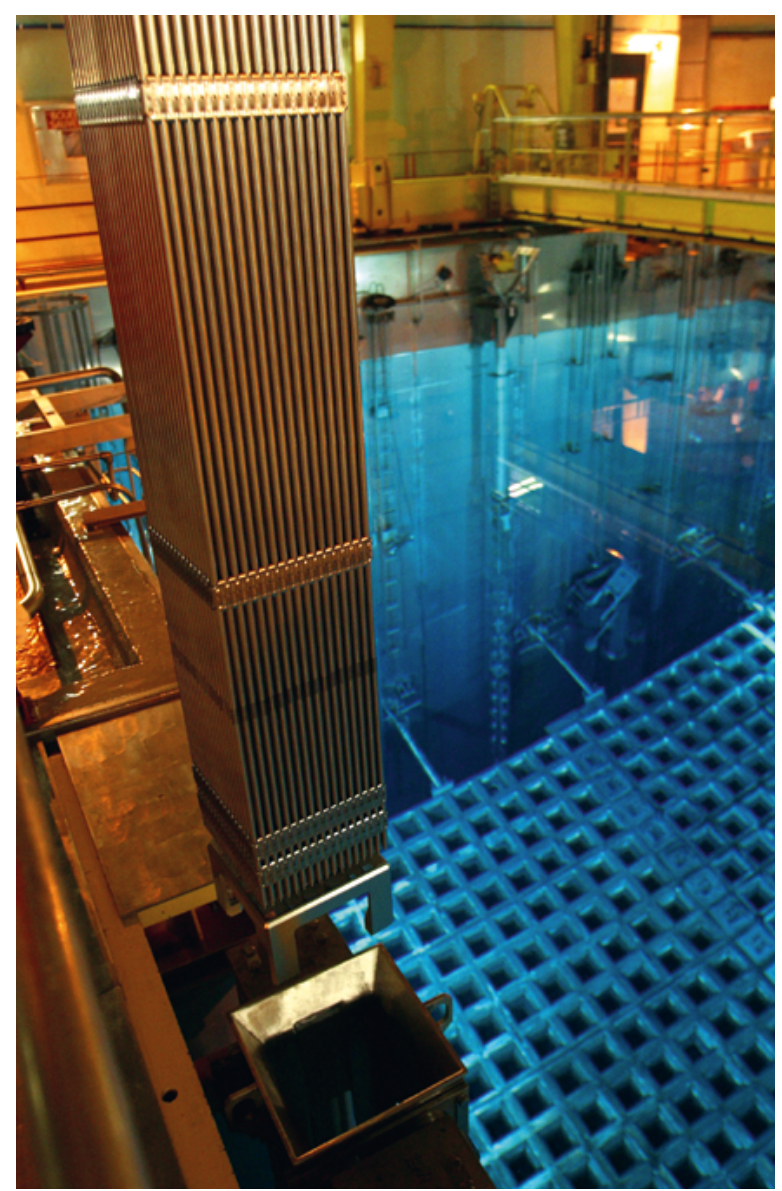

\section{Le modérateur}

La fission d'un noyau de l'uranium 235 ou d'autres atomes fissiles est beaucoup plus facile si les neutrons ont une faible énergie ou vitesse (2 km/s). Or, les neutrons produits par la fission sont émis à très grande vitesse $(20000 \mathrm{~km} / \mathrm{s})$. Il est donc nécessaire de les ralentir en les faisant « rebondir » sur des atomes légers. L'eau du circuit primaire, composée d'atomes d'oxygène mais surtout d'atomes d'hydrogène très légers, permet de ralentir les neutrons. Dans un réacteur à eau légère, l'eau assure à la fois la fonction de caloporteur et de modérateur.

Le contrôle de la réaction nucléaire en chaîne Pour entretenir une réaction en chaîne, il est nécessaire qu'à chaque instant le nombre de neutrons produits par fission soit égal au nombre de neutrons qui disparaissent par capture dans le combustible et les structures du réacteur ou en s'échappant du cœur.

Pour assurer cet équilibre, il est possible d'agir :

- soit sur les grappes de commande que l'on introduit plus ou moins dans le combustible pour modifier le flux de neutrons ;

- soit en injectant dans l'eau du circuit du bore 10 sous la forme d'acide borique qui a la propriété de capturer les neutrons en produisant du tritium.

\section{Évolution du combustible nucléaire} en fonctionnement

Au fur et à mesure que les fissions se produisent dans le cœur, le nombre de noyaux fissiles d'uranium ou de plutonium diminue. Au bout de quelque temps, appelé cycle de fonctionnement (12 à 18 mois), le combustible doit être renouvelé par tiers ou par quart de cœur. Cette opération nécessite l'arrêt du réacteur. 
ANNEXE 8.2

\section{Installation de collecte et de traitement des effluents radioactifs liquides et gazeux}

La maîtrise et l'optimisation des rejets constituent des principes fondamentaux de la conception et du fonctionnement des centrales nucléaires, consistant à mettre en œuvre, à un coût raisonnablement acceptable, les meilleures pratiques d'exploitation et les meilleurs procédés de traitement et de rejet des effluents. La gestion optimisée des effluents et des rejets consiste à :

- réduire à la source la production d'effluents, notamment via une analyse relative aux effluents lors de la préparation des opérations d'exploitation (par exemple lors de vidanges, rinçages), une limitation des fuites par la surveillance et la maintenance préventive des équipements, et une limitation de l'activité des effluents par le maintien en propreté des installations de collecte et de traitement de ces derniers ;

- collecter sélectivement les divers effluents selon leur nature chimique et radiochimique, afin de traiter chacun d'eux le plus efficacement possible, voire dans certains cas les recycler, en maintenant un équilibre entre la production d'effluents et de déchets;

- entreposer et contrôler les effluents avant leur rejet pour garantir le respect des exigences réglementaires.

Ces principes sont illustrés sur les schémas suivants pour les paliers 900 MWe et 1300 MWe.

SCHÉMA DES INSTALLATIONS 900 MW CP1-CP2 E

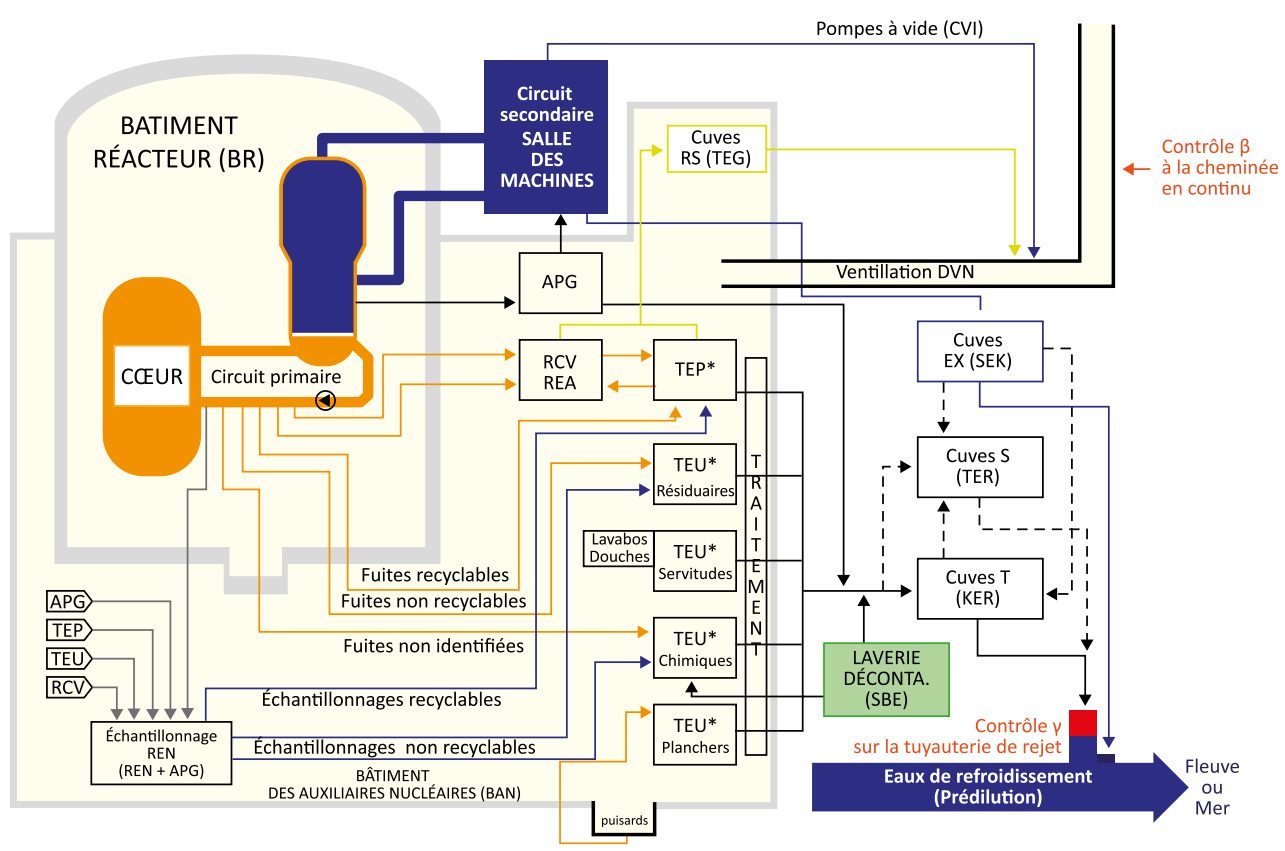




\section{SCHÉMA DES INSTALLATIONS 1300 MW P'4}

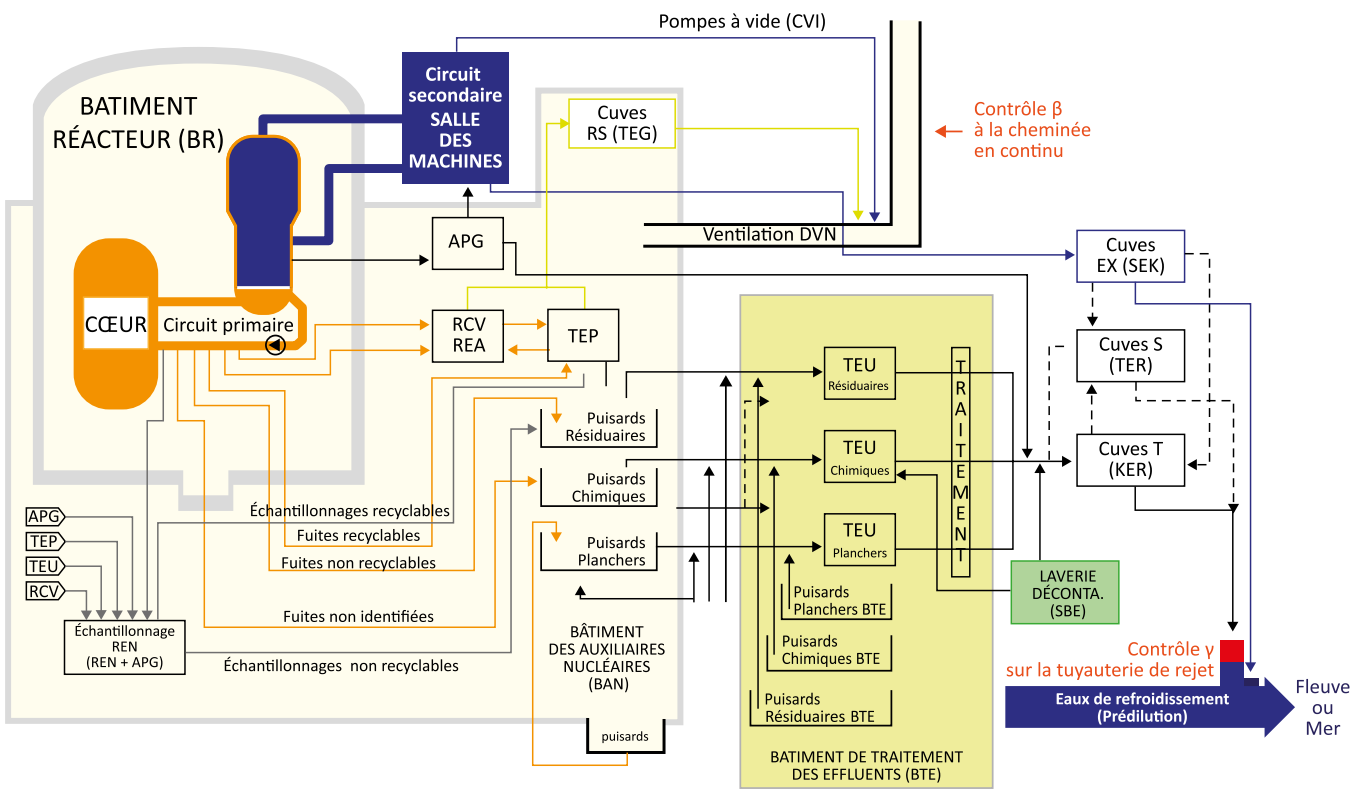




\section{ANNEXE 8.3}

\section{Modalités de rejets des effluents radioactifs liquides - Site sur cours d'eau}

Les figures ci-dessous schématisent les modalités de rejet des effluents radioactifs liquides d'un site en bord de rivière.

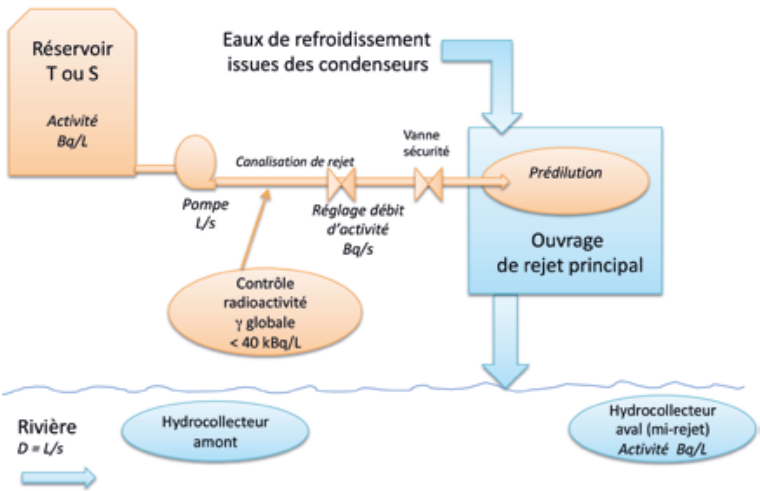

L'hydrocollecteur placé en aval du rejet permet de prélever automatiquement 24 échantillons dans la journée (un échantillon par heure). Le prélèvement réglementaire appelé "mi-rejet" est effectué dans le milieu récepteur à l'instant où la moitié du volume d'effluent contenu dans le réservoir à rejeter a atteint la station multiparamètres aval.

L'heure du «mi-rejet » est déterminée par la formule suivante :

Date et heure mi-rejet $=$ Date et heure du mi-volume du réservoir atteint en sortie du canal de rejet + temps de transit

Le temps de transit de l'effluent entre le point de rejet et l'hydrocollecteur est fonction du débit du cours d'eau ; il est obtenu au moyen d'un abaque.

Avant rejet, le réservoir T d'effluent radioactif est mis en brassage et des analyses sont réalisées sur un échantillon représentatif de l'effluent à rejeter. Une fois les résultats des analyses connus, le rejet est réalisé en ouvrant la vanne de réglage de débit de sorte à respecter la limite de débit d’activité $(\mathrm{Bq} / \mathrm{s})$, le facteur de pré dilution, les flux et concentrations demandés par la réglementation ainsi que les activités volumiques à la station multiparamètres aval.

\begin{tabular}{|l|l|}
\hline $\begin{array}{c}\text { Journée J } \\
\text { avec rejet }\end{array}$ & Heure du mi-rejet \\
\hline $0 \mathrm{~h}$ & $\begin{array}{l}\text { Heure de début } \\
\text { du rejet }\end{array}$ \\
$\begin{array}{l}\text { Sur le prélèvement de } \\
\text { l'échantillon horaire à mi-rejet : } \\
\text { tritium }<280 \mathrm{~Bq} / \mathrm{L} \\
\boldsymbol{\beta} \text { globale }<2 \mathrm{~Bq} / \mathrm{L}\end{array}$
\end{tabular}

Sur l'échantillon aliquote constitué des 24 échantillons horaires de la journée J tritium < $140 \mathrm{~Bq} / \mathrm{L}$,

\section{Journée J \\ sans rejet}




\section{Modalités de rejets des effluents radioactifs liquides - Site marin}

Les figures ci-dessous schématisent les modalités de rejet des effluents radioactifs liquides d'un site en bord de mer ou d'estuaire.

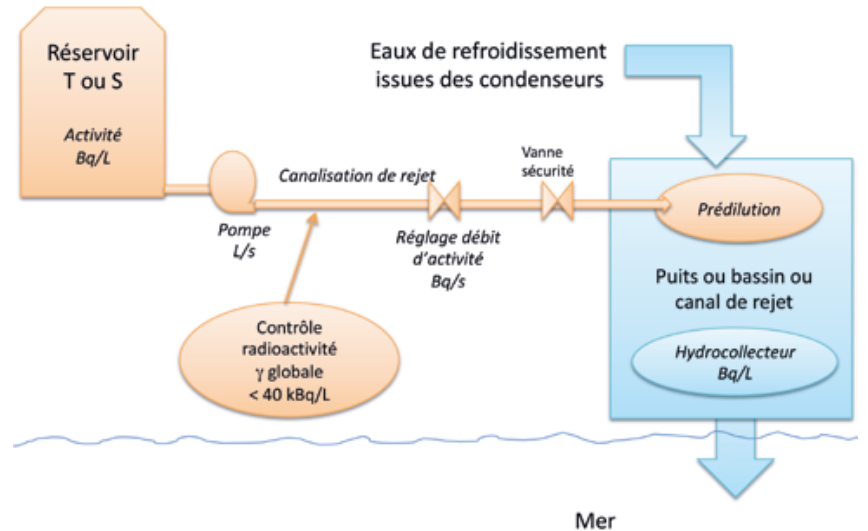

L'hydrocollecteur est placé dans le puits, bassin ou canal de rejet. Il permet de prélever automatiquement 24 échantillons dans la journée (un échantillon par heure). Le prélèvement réglementaire appelé « mi-rejet » y est effectué à l'instant où la moitié du volume d'effluent contenu dans le réservoir à rejeter a été vidangée.

Avant rejet, le réservoir T d'effluent radioactif est mis en brassage et des analyses sont réalisées sur un échantillon représentatif de l'effluent à rejeter. Une fois les résultats des analyses connus, le rejet est réalisé en ouvrant la vanne de réglage de débit de sorte à respecter la limite de débit d'activité (Bq/s), le facteur de pré dilution, les flux et concentrations demandés par la réglementation ainsi que les activités volumiques au niveau de l'hydrocollecteur placé dans le puits, bassin ou canal de rejet en fonction de la configuration du site.

\begin{tabular}{|l|l|l|}
\hline $\begin{array}{c}\text { Journée J } \\
\text { avec rejet }\end{array}$ & Heure du mi-rejet \\
\hline $\begin{array}{l}\text { Heure de début } \\
\text { du rejet }\end{array}$ & \multicolumn{1}{l}{$\begin{array}{l}\text { Sur le prélèvement de } \\
\text { 'échantillon horaire à mi-rejet : } \\
\text { tritium }<1800 \mathrm{~Bq} / \mathrm{L} \\
\beta \text { globale }<18 \mathrm{~Bq} / \mathrm{L}\end{array}$} \\
\hline
\end{tabular}

Sur l'échantillon aliquote constitué des 24 échantillons horaires de la journée J : analyse tritium $<900 \mathrm{~Bq} / \mathrm{L}$

\section{Journée J}

sans rejet

$\mathrm{Oh}$

Sur l'échantillon aliquote constitué des 24 échantillons horaires de la journée J : tritium $<100 \mathrm{~Bq} / \mathrm{L}$ 


\section{Comptabilisation des activités rejetées}

La réglementation relative aux INB, introduite en 1995 par le décret du 4 mai 1995, a créé de nouvelles catégories de radionucléides nécessitant de modifier le système de comptabilisation des effluents radioactifs.

Cette comptabilisation a été mise en application sur le site de Saint-Laurent en 1999 ; elle est utilisée sur l'ensemble des centrales du parc nucléaire d'EDF depuis 2002.

L'ancienne comptabilisation s'appuyait, notamment pour les rejets gazeux, sur des indicateurs globaux (par exemple la mesure bêta gaz globale à la cheminée) ; elle ne permettait pas de distinguer les différents radionucléides entre eux.

La comptabilisation actuelle est fondée sur une analyse radionucléide par radionucléide et fait apparaître dorénavant, de manière explicite, les iodes dans les rejets liquides, le carbone 14 et le nickel 63.

Ces règles s'appuient en premier lieu sur la définition de «spectres de référence », en fonction du type de rejet (liquide : réservoir T, réservoir Ex ; gazeux : rejets permanents et concertés BR et RS). Ces spectres consistent en une liste de radionucléides à identifier par les moyens de mesure adéquats. Toutes les substances figurant dans plus de $90 \%$ des analyses figurent dans cette liste. Des radionucléides, présents à l'état de trace comme l'iode figurent également dans cette liste pour des raisons historiques.

\section{REJETS RADIOACTIFS GAZEUX}

Il a été défini des «spectres de référence » pour chaque type de rejet en distinguant les rejets permanents des rejets concertés.

Gaz rares

- Pour les rejets permanents: ${ }^{133} \mathrm{Xe}$ et ${ }^{135} \mathrm{Xe}$,

- Pour les rejets concertés type BR : ${ }^{41} \mathrm{Ar},{ }^{133} \mathrm{Xe}$ et ${ }^{135} \mathrm{Xe}$,

- Pour les rejets concertés type RS : ${ }^{85} \mathrm{Kr}$, ${ }^{133}$ Xe et ${ }^{131 \mathrm{~m} X e}$ ajouté en 2002.

lodes (ex. halogènes)

- À la cheminée pour l'ensemble des rejets : ${ }^{131} \mid$ et ${ }^{133}$.

Produits de fission ou d'activation émetteurs $\beta / \gamma$ (ex: Aérosols)

- À la cheminée pour l'ensemble des rejets : ${ }^{58} \mathrm{Co},{ }^{60} \mathrm{Co},{ }^{134} \mathrm{Cs},{ }^{137} \mathrm{Cs}$.

Tritium et carbone 14

- À la cheminée pour l'ensemble des rejets.

Nota: Si ces radionucléides ne sont pas détectés, ils sont néanmoins comptabilisés comme ayant une activité volumique égale au « seuil de décision».

\section{REJETS RADIOACTIFS LIQUIDES}

lodes

- Réservoir T : ${ }^{131} 1$,

- Réservoir Ex : l'iode 131 n'est pas comptabilisé si l'activité bêta globale mesurée préalablement au rejet est inférieure à la limite mentionnée dans l'arrêté et si l'analyse de l'échantillon aliquote mensuel ne met pas en évidence d'activité significative de ce radionucléide.

Autres radionucléides émetteurs $\mathrm{b} / \gamma$

- Réservoir T : ${ }^{54} \mathrm{Mn},{ }^{58} \mathrm{Co},{ }^{60} \mathrm{Co},{ }^{110 \mathrm{~m}} \mathrm{Ag},{ }^{124} \mathrm{Sb},{ }^{134} \mathrm{Cs},{ }^{137} \mathrm{Cs}$ plus le ${ }^{133 \mathrm{~m} T e}$ et le ${ }^{125} \mathrm{Sb}$ ajoutés en 2000 et le ${ }^{63} \mathrm{Ni}$ ajouté en 2002,

- Réservoir Ex : les radionucléides du spectre de référence ne sont pas comptabilisés si l'activité bêta globale mesurée préalablement au rejet est inférieure à la limite mentionnée dans l'arrêté et si l'analyse de l'échantillon aliquote mensuel ne met pas en évidence d'activité significative. 


\section{Tritium}

- Réservoirs T : analyse avant rejet,

- Réservoir Ex : analyse avant rejet et comptabilisation sur échantillon aliquote mensuel.

\section{Carbone 14}

- Réservoir T : sur chaque réservoir à partir de l'analyse d'un échantillon représentatif, en fonction des demandes stipulées dans les décisions limites et modalités du site.

Nota : pour les réservoirs T, si les radionucléides de référence ne sont pas détectés, ils sont néanmoins comptabilisés comme ayant une activité volumique égale au " seuil de décision ».

\section{RADIONUCLÉIDES ÉMETTEURS ALPHA D’ORIGINE ARTIFICIELLE DANS LES REJETS LIQUIDES ET GAZEUX}

Le rejet de radionucléides émetteurs alpha d'origine artificielle est interdit. L'absence de radionucléides est déclarée dès lors que le résultat fourni par la mesure est inférieur à un seuil dit de décision fixé dans la réglementation. Ces seuils garantissent, dans le cas le plus pénalisant, un impact dosimétrique potentiel faible vis-à-vis du public (groupes de référence) par rapport aux autres rejets.

\section{En résumé :}

- tous les radionucléides détectés sont systématiquement comptabilisés,

- certains radionucléides fréquents (dits appartenant au spectre de référence) sont comptabilisés au minimum détectable même s'ils n’ont pas été physiquement détectés. 


\section{Fonctionnement d'un aéroréfrigérant}

Les échangeurs utilisés par EDF dans les centrales nucléaires sont de type« humide ». L'échange de chaleur se fait par contact direct entre l'air ambiant et l'eau à refroidir. Dans ce corps d'échange, il se produit deux types d'échanges de chaleur:

- un échange par évaporation d'une partie de l'eau dans l'air (prélèvement de la chaleur latente de vaporisation);

- un échange par convection lié à la différence de température entre l'eau et l'air.

Dans un aéroréfrigérant, environ $75 \%$ de la chaleur est évacuée par évaporation, le restant par convection.

Trois sortes d'aéroréfrigérants sont utilisées dans les centrales refroidies en circuit dit «fermé »:

- aéroréfrigérant à contre-courant. Dans le corps d'échange de l'aéroréfrigérant, l'air circule par tirage naturel de bas en haut et l'eau à refroidir est dispersée dans un packing puis tombe en pluie pour être récupérée dans le bassin d'eau froide (Bugey, Dampierre, Cruas, Civaux, Golfech, Belleville, Chooz, Nogent) ;

- aéroréfrigérant à courant croisé, l'air pénètre horizontalement dans la partie basse de la tour (persienne) et croise l'eau à refroidir qui ruisselle sur un lit de lattes (Cattenom, St-Laurent) ;

- aéroréfrigérant à ventilation forcée ; lorsqu'il n'est pas possible de construire de tours de grande hauteur (paysage), il est nécessaire de recourir à un tirage forcé au moyen de puissants ventilateurs (Chinon).

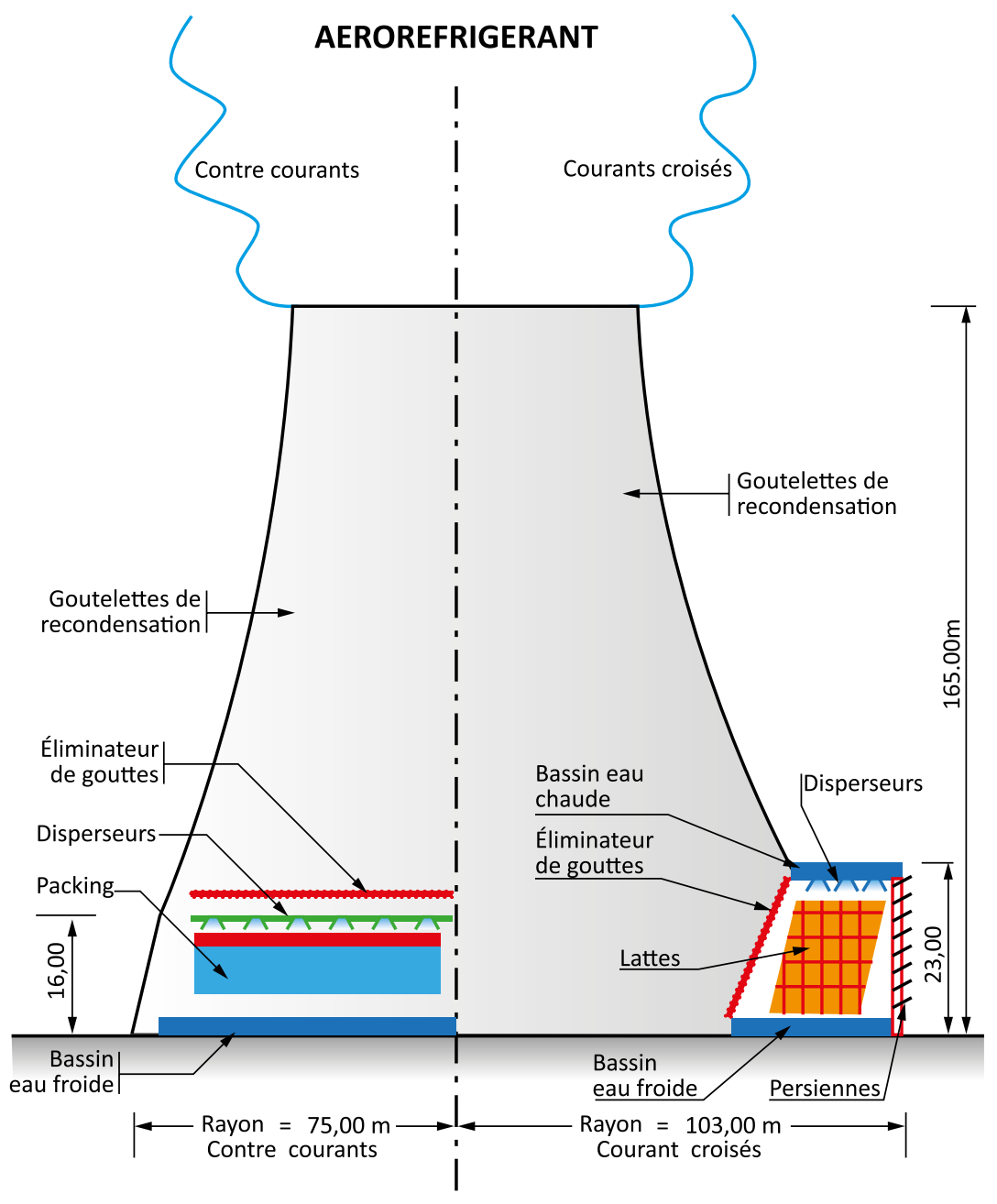




\section{Limites de rejets thermiques des centrales EDF mentionnées dans les décisions ASN}

\section{SITES BORD DE RIVIÈRE}

Limites à respecter en valeurs moyennes journalières sauf prescriptions particulières des décisions limites et modalités (cf. § 5.3)

\begin{tabular}{|c|c|c|c|c|c|}
\hline $\begin{array}{l}\text { COURS } \\
\text { D'EAU }\end{array}$ & CENTRALE & ÉCHAUFFEMENT ${ }^{\circ} \mathrm{C}$ & $\mathrm{T}^{\circ}{ }_{\text {REJET }}{ }^{\circ} \mathrm{C}$ & $\mathrm{T}^{\circ}{ }_{\text {AVAL }}{ }^{\circ} \mathrm{C}$ & RÉFÉRENCE DES DÉCISIONS ASN \\
\hline \multirow{4}{*}{ Loire } & Belleville & $\leq 1^{(1)}$ & - & - & $\begin{array}{c}\text { Décision ASN n 2014-DC-0413 \& } \\
n^{\circ} 2014-D C-0414\end{array}$ \\
\hline & Dampierre & $\leq 1^{(1)}$ & - & - & $\begin{array}{c}\text { Décisions ASN n 2011-DC-0210 \& } \\
n^{\circ} 2011-D C-0211\end{array}$ \\
\hline & Chinon & $\leq 1^{(1)}$ & - & - & $\begin{array}{c}\text { Décision ASN n 2015-DC-0527 \& } \\
n^{\circ} 2015-D C-0528\end{array}$ \\
\hline & St-Laurent & $\leq 1^{(1)}$ & - & - & $\begin{array}{c}\text { Décision ASN n² 2015-DC-0499 \& } \\
n^{\circ} 2015-D C-0498\end{array}$ \\
\hline
\end{tabular}

(1) porté à $1,5^{\circ} \mathrm{C}$ si le débit de la Loire est inférieur à $100 \mathrm{~m}^{3} / \mathrm{s}$ et si la température de la Loire à l'amont est inférieure à $15^{\circ} \mathrm{C}$.

\begin{tabular}{|c|c|c|c|c|c|c|}
\hline $\begin{array}{l}\text { COURS } \\
\text { D'EAU }\end{array}$ & CENTRALE & ÉCHAUFFEMENT ${ }^{\circ} \mathrm{C}$ & $\mathrm{T}^{\circ}{ }_{\text {REJET }}{ }^{\circ} \mathrm{C}$ & $\mathrm{T}_{\text {AVAL }}^{\circ}{ }^{\circ} \mathrm{C}$ & PÉRIODE & $\begin{array}{l}\text { RÉFÉRENCE DES } \\
\text { DÉCISIONS ASN }\end{array}$ \\
\hline \multirow{4}{*}{ Vienne } & \multirow{4}{*}{ Civaux } & $\leq 2^{(1)}$ & - & $\leq 25^{(1)}$ & - & \multirow{4}{*}{$\begin{array}{c}\text { Décisions ASN } \\
\text { n² 2009-DC-0139 \& } \\
\text { n² 2009-DC-0138 }\end{array}$} \\
\hline & & $\leq 0^{(2)}$ & - & $\leq \mathrm{T}_{\text {amont }}^{\circ}$ & - & \\
\hline & & $\leq 1^{(3)}$ & - & $\leq 28$ & - & \\
\hline & & $\leq 3^{(4)}$ & & $\leq 25^{(4)}$ & $\begin{array}{l}\text { Étiage exc. d'hiver } \\
\text { du 01/11 au 30/04 }\end{array}$ & \\
\hline
\end{tabular}

(1) Si $T_{\text {amont }}^{\circ}<25^{\circ} \mathrm{C}$; (2) Si $T_{\text {amont }}^{\circ} \geq 25^{\circ} \mathrm{C}$ et si aéroréfrigérant des purges disponible; (3) Si $T_{\text {amont }}^{\circ} 25$ et si indisponibilité fortuite de l'aéroréfrigérant des purges et sous conditions particulières; (4) Après accord du directeur général de l'ASN.

\begin{tabular}{|c|c|c|c|c|c|c|}
\hline $\begin{array}{l}\text { COURS } \\
\text { D'EAU }\end{array}$ & CENTRALE & ÉCHAUFFEMENT ${ }^{\circ} \mathrm{C}$ & $\mathrm{T}_{\text {REJET }}^{\circ}{ }^{\circ} \mathrm{C}$ & $\mathrm{T}^{\circ}{ }_{\text {AVAL }}{ }^{\circ} \mathrm{C}$ & PÉRIODE & $\begin{array}{l}\text { RÉFÉRENCE DES } \\
\text { DÉCISIONS ASN }\end{array}$ \\
\hline \multirow{8}{*}{ Rhône } & \multirow{3}{*}{ Bugey } & $\leq 5$ & - & $\leq 26$ & Du 01/05 au 15/09 & \multirow{3}{*}{$\begin{array}{c}\text { Décision ASN } \\
\mathrm{n}^{\circ} 2014-\mathrm{DC}-0443 \text { \& } \\
\mathrm{n}^{\circ} \text { 2014-DC-0442 }\end{array}$} \\
\hline & & $\leq 7$ & - & $\leq 24$ & Du 16/09 au 30/04 & \\
\hline & & $\leq 1^{(1)}$ & - & $\leq 27$ & $\begin{array}{l}\text { Conditions climatiques } \\
\text { exceptionnelles }\end{array}$ & \\
\hline & \multirow{3}{*}{ St-Alban } & $\leq 3$ & - & $\leq 28$ & Du 16/05 au 30/09 & \multirow{3}{*}{$\begin{array}{c}\text { Décision ASN } \\
n^{\circ} \text { 2014-DC-0469 } \\
\& n^{\circ} \text { 2014-DC-0470 }\end{array}$} \\
\hline & & $\leq 4$ & - & $\leq 26$ & Du $01 / 10$ au 15/05 & \\
\hline & & $\begin{array}{c}\leq 3 \text { ou } 4 \text { selon } \\
\text { la période }\end{array}$ & - & $\leq 29$ & $\begin{array}{c}\text { Conditions climatiques } \\
\text { exceptionnelles }\end{array}$ & \\
\hline & \multirow[b]{2}{*}{ Cruas } & $\leq 1$ & - & $\leq 28$ & - & \multirow{2}{*}{$\begin{array}{c}\text { Décision ASN } \\
n^{\circ} 2016-D C-0548 \\
\& n^{\circ} 2016-D C-0549\end{array}$} \\
\hline & & - & - & $\leq 29$ & $\begin{array}{c}\text { Conditions climatiques } \\
\text { exceptionnelles }\end{array}$ & \\
\hline \multirow{3}{*}{$\begin{array}{l}\text { Rhône } \\
\text { Canal de } \\
\text { Donzère- } \\
\text { Mondragon }\end{array}$} & \multirow{3}{*}{ Tricastin } & $\leq 4^{(3)}$ & - & $\leq 28$ & - & \multirow{3}{*}{$\begin{array}{c}\text { Décisions ASN } \\
\mathrm{n}^{\circ} 2008-\mathrm{DC}-0102 \text { \& } \\
\mathrm{n}^{\circ} \text { 2008-DC-0101 }\end{array}$} \\
\hline & & $\leq 6^{(4)}$ & - & $\leq 28$ & - & \\
\hline & & $\leq 3$ & - & $\leq 29$ & $\begin{array}{c}\text { Conditions climatiques } \\
\text { exceptionnelles }\end{array}$ & \\
\hline
\end{tabular}

(1) Pour $26^{\circ} \mathrm{C}<\operatorname{Taval}<27^{\circ} \mathrm{C}$, les tranches 2 et 3 sont mises à l'arrêt; (2) Les conditions climatiques exceptionnelles ne sont applicables que si le réseau de transport d'électricité (RTE) requiert le fonctionnement de la centrale nucléaire à un niveau de puissance minimal, ou si l'équilibre entre la consommation et la production d'électricité nécessite son fonctionnement; (3) Si le débit canal $>480 \mathrm{~m}^{3} / \mathrm{s}$; (4) Si le débit canal $<480 \mathrm{~m}^{3} / \mathrm{s}$. 


\begin{tabular}{|c|c|c|c|c|c|c|}
\hline $\begin{array}{l}\text { COURS } \\
\text { D'EAU }\end{array}$ & CENTRALE & ÉCHAUFFEMENT C & $\mathrm{T}^{\circ}{ }_{\text {REJET }}{ }^{\circ} \mathrm{C}$ & $\mathrm{T}^{\circ}{ }_{\text {AVAL }}{ }^{\circ} \mathrm{C}$ & CONDITIONS & $\begin{array}{l}\text { RÉFÉRENCE DES } \\
\text { DÉCISIONS ASN }\end{array}$ \\
\hline \multirow{4}{*}{$\begin{array}{l}\text { Moselle } \\
\text { Retenue du } \\
\text { Mirgenbach }\end{array}$} & \multirow{4}{*}{ Cattenom } & $\leq 1,5$ & $\leq 28$ & - & $\mathrm{Si} \mathrm{T}_{\text {amont }} \leq 28^{\circ} \mathrm{C}$ & \multirow{4}{*}{$\begin{array}{c}\text { Décision ASN } \\
\mathrm{n}^{\circ} \text { 2014-DC-0416 } \\
\& \mathrm{n}^{\circ} 2014-\mathrm{DC}-0415\end{array}$} \\
\hline & & 0 & - & - & $28^{\circ} \mathrm{C}<\mathrm{T}_{\text {amont }}^{\mathrm{Si}}<30^{\circ} \mathrm{C}$ & \\
\hline & & 0 & $\begin{array}{c}\text { Rejets } \\
\text { interdits }\end{array}$ & - & $\mathrm{Si} \mathrm{T}_{\text {amont }}>30^{\circ} \mathrm{C}$ & \\
\hline & & 0 & - & - & $\begin{array}{l}\text { Conditions clima- } \\
\text { tiques exception- } \\
\text { nelles }{ }^{(1)}\end{array}$ & \\
\hline
\end{tabular}

(1) L'entrée en conditions climatiques exceptionnelles n'est applicable que si le réseau de transport d'électricité (RTE) requiert le fonctionnement de la centrale nucléaire à un niveau de puissance minimal, ou si l'équilibre entre la consommation et la production d'électricité nécessite son fonctionnement.

\begin{tabular}{|l|c|c|c|c|c|}
\hline COURS D'EAU & CENTRALE & ÉCHAUFFEMENT ${ }^{\circ} \mathrm{C}$ & $\mathrm{T}^{\circ}{ }_{\text {REJET }}^{\circ} \mathrm{C}$ & T $^{\circ}{ }_{\text {AVAL }}^{\circ} \mathrm{C}$ & $\begin{array}{c}\text { RÉFÉRENCE } \\
\text { DES DÉCISIONS ASN }\end{array}$ \\
\hline Meuse & Chooz & $\leq 3^{(1)}$ & - & $\leq 28^{(1)}$ & $\begin{array}{c}\text { Décisions ASN } n^{\circ} 2009-D C- \\
0165 \& n^{\circ} 2009-D C-0164\end{array}$ \\
\hline
\end{tabular}

(1) 5 jours par an du $1 / 05$ au $30 / 09$ si $T^{\circ}{ }_{\text {amont }} \geq 26^{\circ} \mathrm{C}$, on pourra avoir DT max $2{ }^{\circ} \mathrm{C}$ et T aval max $<30^{\circ} \mathrm{C}$.

\begin{tabular}{|c|c|c|c|c|c|c|}
\hline $\begin{array}{l}\text { COURS } \\
\text { D'EAU }\end{array}$ & CENTRALE & ÉCHAUFFEMENT ${ }^{\circ} \mathrm{C}$ & $\mathrm{T}^{\circ}{ }_{\text {REJet }}{ }^{\circ} \mathrm{C}$ & $\mathrm{T}_{\text {AVAL }}^{\circ}{ }^{\circ} \mathrm{C}$ & PÉRIODE & $\begin{array}{l}\text { RÉFÉRENCE DES } \\
\text { DÉCISIONS ASN }\end{array}$ \\
\hline \multirow[b]{3}{*}{ Seine } & \multirow[b]{3}{*}{ Nogent } & $\leq 3$ & - & $\leq 28$ & & \multirow{3}{*}{$\begin{array}{c}\text { Arrêté min. } \\
\text { du 29/12/2004 }\end{array}$} \\
\hline & & $\leq 4^{(1)}$ & & & & \\
\hline & & $\leq 1,5$ & - & $\leq 30$ & $\begin{array}{c}\text { Conditions } \\
\text { climatiques }^{(2)(3)} \\
\text { exceptionnelles }\end{array}$ & \\
\hline
\end{tabular}

(1) $\Delta T 4^{\circ} \mathrm{C}$ de novembre à février si Q Seine $<20 \mathrm{~m}^{3} / \mathrm{s}$; (2) autorisé $2 \%$ du temps sur une année calendaire en situation climatique exceptionnelle et sous conditions particulières; (3) L'utilisation des conditions climatiques exceptionnelles sera limitée aux situations où le réseau de transport d'électricité (RTE) requiert le fonctionnement de la centrale nucléaire de Nogent à un niveau de puissance minimal ou pour lesquelles l'équilibre entre la consommation et la production d'électricité nécessite le fonctionnement de la centrale de Nogent.

\begin{tabular}{|c|c|c|c|c|c|c|}
\hline $\begin{array}{l}\text { COURS } \\
\text { D'EAU }\end{array}$ & CENTRALE & ÉCHAUFFEMENT ${ }^{\circ} \mathrm{C}$ & $\mathrm{T}_{\text {REJET }}^{\circ}{ }^{\circ} \mathrm{C}$ & $\mathrm{T}^{\circ}{ }_{\text {AVAL }}{ }^{\circ} \mathrm{C}$ & PÉRIODE & $\begin{array}{l}\text { RÉFÉRENCE DES } \\
\text { AUTORISATIONS } \\
\text { DE REJET }\end{array}$ \\
\hline \multirow[b]{3}{*}{ Garonne } & \multirow[b]{3}{*}{ Golfech } & $\leq 1,25$ & - & $\leq 28$ & De 01/06 au 30/09 & \multirow{3}{*}{$\begin{array}{l}\text { Arrêté ministériel du } \\
\text { 18/09/2006 }\end{array}$} \\
\hline & & $\leq 2$ & - & $\leq 28$ & Du $01 / 10$ au $31 / 05$ & \\
\hline & & $\leq 1,25$ & - & $\leq 30$ & $\begin{array}{l}\text { Conditions clima- } \\
\text { tiques exception- } \\
\text { nelles }^{(1)}\end{array}$ & \\
\hline
\end{tabular}

(1) I'utilisation des valeurs sera limitée aux situations où le réseau de transport d'électricité (RTE) requiert le fonctionnement de la centrale nucléaire de Golfech à un niveau de puissance minimal ou quand l'équilibre entre la consommation et la production d'électricité nécessite le fonctionnement de la centrale nucléaire de Golfech.

L'utilisation est conditionnée à des besoins du réseau, avec: si T aval $\geq 29^{\circ} \mathrm{C}=>$ lâcher d'eau de $3 \mathrm{~m}^{3} / \mathrm{s}$ dans une limite de 3 millions de $\mathrm{m}^{3}$. Règles des lâchés d'eau en situation exceptionnelle : barrage de St Peyres privilégié dans la limite de 1 million de $m^{3}$, les retenues de l'Ariège et de Lunax venant en complément dans la limite de 2 millions de $\mathrm{m}^{3}$. 
SITE SUR ESTUAIRE ASSIMILÉ À UN SITE BORD DE MER

\begin{tabular}{|c|c|c|c|c|c|c|}
\hline ESTUAIRE & CENTRALE & $\begin{array}{l}\text { ÉCHAUFFEMENT } \\
\text { PRISE - REJET }{ }^{\circ} \mathrm{C}\end{array}$ & $\mathrm{T}^{\circ}{ }_{\text {REJET }}{ }^{\circ} \mathrm{C}$ & $\mathrm{T}^{\circ}{ }_{\text {AVAL }}{ }^{\circ} \mathrm{C}$ & PÉRIODE & $\begin{array}{c}\text { RÉFÉRENCE } \\
\text { DES AUTORISA- } \\
\text { TIONS DE REJET }\end{array}$ \\
\hline \multirow{2}{*}{ Gironde } & \multirow{2}{*}{ Blayais } & $\leq 11$ & $\leq 36,5$ & $\leq 30$ & Du $15 / 05$ au $15 / 10$ & \multirow{2}{*}{$\begin{array}{c}\text { Arrêté ministériel } \\
\text { du 18/09/2003 }\end{array}$} \\
\hline & & $\leq 11$ & $\leq 30$ & $\leq 30$ & Du $16 / 10$ au $15 / 05$ & \\
\hline
\end{tabular}

\section{SITES BORD DE MER}

\begin{tabular}{|c|c|c|c|c|c|c|}
\hline MER & CENTRALE & ÉCHAUFFEMENT ${ }^{\circ} \mathrm{C}$ & $\begin{array}{l}\mathrm{T}^{\circ} \\
\mathrm{MAEJET}^{\circ} \mathrm{C}\end{array}$ & $\mathrm{MAX}^{\mathrm{T}^{\circ} \mathrm{C}}$ & PÉRIODE & $\begin{array}{l}\text { RÉFÉRENCE DES } \\
\text { DÉCISIONS ASN }\end{array}$ \\
\hline \multirow{11}{*}{ Manche } & \multirow{2}{*}{ Flamanville 1-2 } & $\leq 15$ & $\leq 35$ & $\leq 30$ & Du 01/06 au 31/10 & \multirow{5}{*}{$\begin{array}{c}\text { Décision ASN } \\
\mathrm{n}^{\circ} \text { 2018-DC-0639 } \\
\& \mathrm{n}^{\circ} 2018-\mathrm{DC}-0640\end{array}$} \\
\hline & & $\leq 15$ & $\leq 30$ & $\leq 30$ & Du $1 / 11$ au $31 / 05$ & \\
\hline & \multirow{2}{*}{ Flamanville 3} & $\leq 14$ & $\leq 35$ & $\leq 30$ & Du $01 / 06$ au $31 / 10$ & \\
\hline & & $\leq 14$ & $\leq 30$ & $\leq 30$ & Du $1 / 11$ au $31 / 05$ & \\
\hline & $\begin{array}{c}\text { Flamanville } \\
1-2-3\end{array}$ & $\leq 21$ & - & - & $\begin{array}{c}\text { Situation } \\
\text { particulière }\end{array}$ & \\
\hline & \multirow{3}{*}{ Paluel } & $\leq 15$ & $\leq 35$ & $\leq 30$ & Du $01 / 06$ au $31 / 10$ & \multirow{3}{*}{$\begin{array}{c}\text { Décision ASN } \\
n^{\circ} 2019-D C-067 \& \\
n^{\circ} 2019-D C-0677\end{array}$} \\
\hline & & $\leq 15$ & $\leq 30$ & $\leq 30$ & Du 1/11 au 31/05 & \\
\hline & & $\leq 21$ & - & & 20 jours par an & \\
\hline & \multirow{3}{*}{ Penly } & $\leq 15$ & $\leq 35$ & $\leq 30$ & Du 01/06 au 31/10 & \multirow{3}{*}{$\begin{array}{c}\text { Décisions ASN } \\
\text { n 2008-DC-0090 \& } \\
\text { n² 2008-DC-0089 }\end{array}$} \\
\hline & & $\leq 15$ & $\leq 30$ & $\leq 30$ & Du 1/11 au 31/05 & \\
\hline & & $\leq 21$ & & & $\begin{array}{l}\text { Situation particu- } \\
\text { lière }^{(1)}\end{array}$ & \\
\hline \multirow{2}{*}{$\begin{array}{l}\text { Mer du } \\
\text { Nord }\end{array}$} & \multirow[b]{2}{*}{ Gravelines } & $\leq 12$ & $\leq 35$ & $\leq 30$ & Du $01 / 06$ au $31 / 10$ & \multirow{2}{*}{$\begin{array}{c}\text { Décision ASN } \\
n^{\circ} 2018-D C-0646 \\
\& n^{\circ} 2018-D C-0647\end{array}$} \\
\hline & & $\leq 12$ & $\leq 30$ & $\leq 30$ & Du $01 / 11$ au $31 / 05$ & \\
\hline
\end{tabular}

(1) Exemples : indisponibilité d'une pompe de circulation alimentant les condenseurs; nettoyage de la station de pompage... La durée cumulée de ces situations particulières n'excède pas vingt jours par an. 

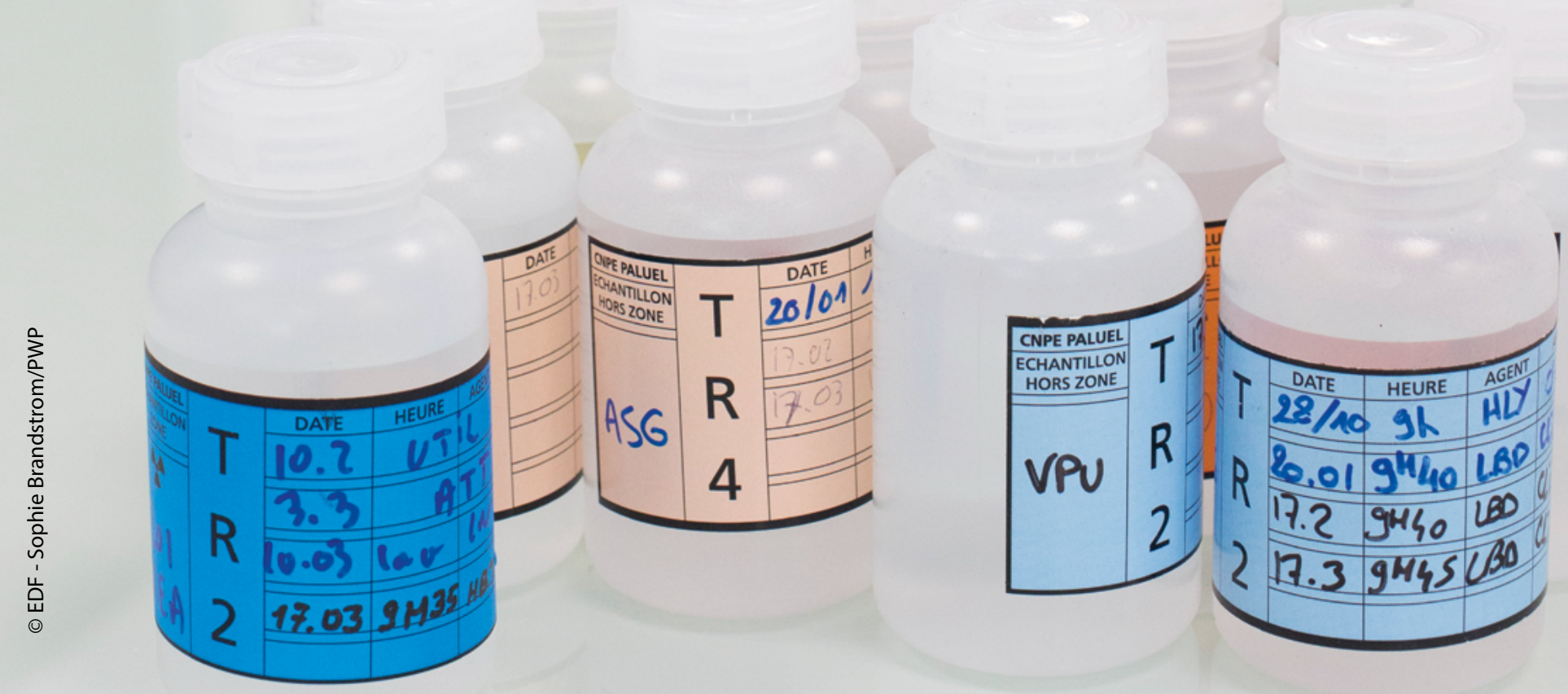